Electronic Journal of Statistics

Vol. 15 (2021) 5696-5757

ISSN: $1935-7524$

https://doi.org/10.1214/21-EJS1919

\title{
Inference for high-dimensional varying-coefficient quantile regression*
}

\author{
Ran Dai \\ Department of Biostatistics, University of Nebraska Medical Center, USA \\ e-mail: ran.dai@unmc.edu
}

Mladen Kolar

Booth School of Business, University of Chicago, USA

e-mail: mkolar@chicagobooth.edu

\begin{abstract}
Quantile regression has been successfully used to study heterogeneous and heavy-tailed data. Varying-coefficient models are frequently used to capture changes in the effect of input variables on the response as a function of an index or time. In this work, we study high-dimensional varying-coefficient quantile regression models and develop new tools for statistical inference. We focus on development of valid confidence intervals and honest tests for nonparametric coefficients at a fixed time point and quantile, while allowing for a high-dimensional setting where the number of input variables exceeds the sample size. Performing statistical inference in this regime is challenging due to the usage of model selection techniques in estimation. Nevertheless, we can develop valid inferential tools that are applicable to a wide range of data generating processes and do not suffer from biases introduced by model selection. We performed numerical simulations to demonstrate the finite sample performance of our method, and we also illustrated the application with a real data example.
\end{abstract}

MSC2020 subject classifications: Primary $62 \mathrm{G} 08$.

Keywords and phrases: High-dimensional inference, quantile regression, varying-coefficient regression.

Received July 2020.

\section{Introduction}

Most statistical work on regression problems has centered on the problem of modeling the mean of a response variable $Y \in \mathbb{R}$ as a function of a feature vector $X \in \mathbb{R}^{p}$. Under some assumptions, for instance assuming homoscedastic Gaussian noise, modeling the mean is sufficient to capture the entire distribution of $Y$ conditioned on the observed features $X=x$. In many applications, however, where these types of assumptions may not be appropriate, it is often far more meaningful to model the median (or some other specified quantile) of $Y$ given the observed feature vector $X$. In particular, in applications where we

${ }^{*}$ This work is partially supported by the William S. Fishman Faculty Research Fund at the University of Chicago Booth School of Business. This work was completed in part with resources supported by the University of Chicago Research Computing Center. 
are interested in extreme events - for instance, modeling changes in stock prices, or modeling birth weight of infants - modeling, e.g., the $90 \%$ quantile may be far more informative than modeling the mean. In other settings, the mean is overly sensitive to outliers, while the median or some other quantile does not have this disadvantage. Fixing $\tau$ to be the desired quantile (e.g., $\tau=0.5$ for the median), we write $q(x ; \tau)$ to be the $\tau$ th quantile for the variable $Y$ conditional on observing $X=x$, that is, $q(x ; \tau)$ is the function that satisfies

$$
q(x ; \tau)=\inf _{q \in \mathbb{R}}\{\mathbb{P}\{Y \leq q \mid X=x\} \geq \tau\} .
$$

In this paper, we are interested in a high-dimensional setting, where the vector $X$ includes an extremely large number of measured features - perhaps larger than the sample size itself. A linear model, $q(x ; \tau)=x^{\top} \beta(\tau)$, may be considered to be a reasonable approximation in many settings, but if the measurements are gathered across different points in time, the effect of the features on the response $Y$ may not be stationary. To achieve broader applicability of our model, we are furthermore interested in models with time-varying coefficients for the $\tau$ th quantile for the variable $Y$ conditional on observing $X=x$ at index $U=u$,

$$
q(x ; \tau, u)=\inf _{q \in \mathbb{R}}\{\mathbb{P}\{Y \leq q \mid X=x, U=u\} \geq \tau\},
$$

where $x \in \mathbb{R}^{p}$ is the feature vector as before, $\tau \in(0,1)$ is the desired quantile, and $u \in \mathcal{U}$ represents the time of the measurement or any other index variable that captures non-stationary effects of the features - for example, $u$ may be used to encode spatial location. We assume that $q(x ; \tau, u)$ approximately follows a linear model $x^{\top} \beta(\tau, u)$.

Fixing a quantile $\tau$ and a time point (or index value) $u$, we are interested in performing inference on a low-dimensional subset of coefficients of interest, $\beta_{\mathcal{A}}(\tau, u)$ for some fixed subset $\mathcal{A} \subset\{1, \ldots, p\}$. Specifically, we want to construct confidence intervals for these parameters or test null hypotheses such as $H_{0}$ : $\beta_{j}(\tau, u)=0, \forall j \in \mathcal{A}$. In practice, we may have in mind some particular features of interest, and the other features are confounding variables that we need to control for; or, we may be interested in testing each of the $p$ features individually, cycling through them in turn and treating the others as confounders.

Prior work Our work is related to the literature on high-dimensional inference, varying-coefficient models, and quantile regression. Statistical inference for parameters in high-dimensional models has received a lot of attention recently. For example, in the $\ell_{1}$-regularized linear regression model (LASSO) Tibshirani (1996) one can quantify the uncertainty about the unknown parameters by debiasing the estimator (Zhang and Zhang, 2013; van de Geer and Bühlmann, 2013; Javanmard and Montanari, 2013b,a) or using a double LASSO selection procedure (Belloni and Chernozhukov, 2013). Extensions to generalized linear models were investigated in Belloni et al. (2016b), van de Geer et al. (2014), and Farrell (2015). Meinshausen (2015) studied construction of one-sided confidence intervals for groups of variables under weak assumptions on the design 
matrix. Lockhart et al. (2014) studied significance of the input variables that enter the model along the lasso path. Lee et al. (2013) and Taylor et al. (2014) performed post-selection inference conditional on the selected model. Kozbur (2013) extended approach developed in Belloni et al. (2013a) to a nonparametric regression setting, where a pointwise confidence interval is obtained based on the penalized series estimator, while Lu et al. (2020) studied a kernel-sieve hybrid estimator for inference in sparse additive models. Yu et al. (2020a) considered testing in high-dimensional parametric models with cone constraints. Hypothesis testing and confidence intervals for low-dimensional parameters in graphical models were studied in (Ren et al., 2015; Wang and Kolar, 2014; Janková and van de Geer, 2015; Janková and van de Geer, 2017), elliptical copula models (Barber and Kolar, 2018; Lu et al., 2018), Markov networks (Wang and Kolar, 2016; Yu et al., 2016, 2020b), differential networks (Xia et al., 2015; Belilovsky et al., 2016; Liu, 2017; Kim et al.), and networks of point processes (Wang et al., 2020). Varying-coefficient models were introduced as a general framework that tied together generalized additive models and dynamic generalized linear models Hastie and Tibshirani (1993). Estimation and inference for varying coefficient models in the mean have been widely studied. See, for example, Fan and Zhang (2000), Hoover et al. (1998), Zhang et al. (2002), Huang et al. (2004), Na et al. (2019), and Na and Kolar (2021). Quantile regression was studied in the presence of outliers and non-normal errors in Koenker (1984), while quantile regression with time-varying coefficient models was studied in, for example, Kim (2007) and Kai et al. (2011). Statistical inference for high-dimensional linear quantile regression was studied in Belloni et al. (2013b, 2015, 2016a); Bradic and Kolar (2017) and a closely related problem of inference in composite quantile regression was investigated in Zhao et al. (2014). Tang et al. (2013) studied estimation of quantile varying-coefficient models in a high-dimensional setting. However, how to perform statistical inference for high-dimensional varying coefficient models remains an open question.

Our contribution Below, we summarize the main contributions of this work.

- We propose several approaches for constructing valid post-selection confidence intervals for the varying-coefficient quantile regression model. These approaches are asymptotically equivalent and rely on finding an approximate root of the decorrelated score. To make the construction computationally feasible with the non-differentiable loss that is used in quantile regression, we rely on a one-step approximation and reparameterization.

- We provide the asymptotic normality results for the proposed estimators. Establishing this results requires a novel analysis that generalizes the existing techniques. Specifically, we carefully overcome the challenges that arise from the non-differentiable loss used in quantile regression, the bias from the penalized regression to handle the high dimensionality, and the bias from linear approximation to handle the nonparametric component in appearing in varying coefficient models.

- We use extensive simulation studies and real data analysis to demonstrate 
the finite sample performance of our proposed estimators.

\section{Preliminaries}

In this section, we carefully develop background necessary to understand the algorithms that are presented in the subsequent section. In Section 2.1 we provide a brief overview of estimation in the varying-coefficient quantile regression model. Next, we illustrate the challenges in the high-dimensional inference in Section 2.2. In Section 2.3, we describe the decorrelated score method that can be used for high-dimensional inference when the loss function is twice differentiable. Finally, we modify the decorrelated score method to suit the non-differentiable setting of varying-coefficient quantile regression and sketch the main steps of the analysis in Section 2.4. Note that the results in Section 2.1-2.3 are not new and are presented for ease of readability.

\subsection{Varying-coefficient quantile regression}

For a random variable $Y \in \mathbb{R}$, its $\tau$-quantile can be equivalently described as the value $q$ that minimizes the expectation $\mathbb{E}\left[\tau \cdot(Y-q)_{+}+(1-\tau) \cdot(Y-q)_{-}\right]$(for any $t \in \mathbb{R}$, we write $t_{+}=\max \{t, 0\}$ and $\left.t_{-}=\max \{-t, 0\}\right)$. For a linear quantile regression problem, at a particular value of the index variable $u \in \mathcal{U} \subseteq \mathbb{R}$, we are therefore interested in estimating

$$
\beta(\tau, u)=\underset{b \in \mathbb{R}^{p}}{\arg \min } \mathbb{E}\left[\tau \cdot\left(Y-X^{\top} b\right)_{+}+(1-\tau) \cdot\left(Y-X^{\top} b\right)_{-} \mid U=u\right],
$$

where the expectation is taken over a draw of the random pair $(X, Y)$ when the index variable is equal to $U=u$ (in other words, we can think of drawing the triplet $(X, Y, U)$ and conditioning on the event $U=u)$.

Of course, we cannot compute this expected value or even obtain an unbiased estimate, unless by some chance our training data contains many data points $\left(x_{i}, y_{i}, u_{i}\right)$ with $u_{i}=u$. Instead, by assuming that $\beta(\tau, u)$ is reasonably smooth with respect to the index variable $u \in \mathcal{U}$, we can use a kernel method, and approximate the expected value in (1) with

$$
\sum_{i=1}^{n} w_{i} \cdot\left[\tau \cdot\left(y_{i}-x_{i}^{\top} b\right)_{+}+(1-\tau) \cdot\left(y_{i}-x_{i}^{\top} b\right)_{-}\right]
$$

where the weights are given as $w_{i}=(n h)^{-1} K\left(h^{-1}\left(u_{i}-u\right)\right)$, the function $K(\cdot)$ is the kernel function, and $h$ is the bandwidth. This approximation can be interpreted as assuming that $\beta(\tau, u)$ is locally approximately constant for values $u_{i} \approx u$, and thus defines a loss function on the sampled data that would hopefully be minimized at some $b(\tau, u) \approx \beta(\tau, u)$, but would suffer bias from the error in this approximation. We can reduce the approximation bias by instead treating $\beta(\tau, u)$ as locally approximately linear for values $u_{i} \approx u$, that is,

$$
x_{i}^{\top} \beta\left(\tau, u_{i}\right) \approx x_{i}^{\top} \beta(\tau, u)+\left(u_{i}-u\right) \cdot x_{i}^{\top} \nabla_{u} \beta(\tau, u) .
$$


Defining $\Gamma_{i}=\left(x_{i}^{\top},\left(u_{i}-u\right) \cdot x_{i}^{\top}\right)^{\top} \in \mathbb{R}^{2 p}$ for each observation $i=1, \ldots, n$, this yields a new loss function,

$$
\mathcal{L}(b)=\sum_{i=1}^{n} w_{i} \cdot\left[\tau \cdot\left(y_{i}-\Gamma_{i}^{\top} b\right)_{+}+(1-\tau) \cdot\left(y_{i}-\Gamma_{i}^{\top} b\right)_{-}\right]=\sum_{i=1}^{n} w_{i} \cdot \rho_{\tau}\left(y_{i}-\Gamma_{i}^{\top} b\right)
$$

where the function $\rho_{\tau}(v)=v(\tau-\mathbb{1}\{v<0\})$. We are now interested in minimizing (2) over a larger parameter vector, $b=\left(b_{0}^{\top}, b_{1}^{\top}\right)^{\top} \in \mathbb{R}^{2 p}$, where $b_{0}, b_{1} \in \mathbb{R}^{p}$. We would expect the minimum to be attained at some $b^{\star}=\left(b_{0}^{\star \top}, b_{1}^{\star \top}\right)^{\top} \approx$ $\left(\beta(\tau, u)^{\top}, \nabla_{u} \beta(\tau, u)^{\top}\right)^{\top}$ if the local linear approximation is sufficiently accurate. Note that we omit the indices $(\tau, u)$ to simplify the notation, as they are fixed.

In a high dimensional setting where the dimension of the covariates $X, p$, is growing faster than the sample size $n$, we use a group $\ell_{1}$-penalty to estimate $b^{\star}$ under the assumption that the coefficient functions are approximately sparse. In particular, we minimize the following optimization program

$$
\widehat{b}=\underset{b \in \mathbb{R}^{2 p}}{\arg \min } \sum_{i=1}^{n} w_{i} \cdot \rho_{\tau}\left(y_{i}-\Gamma_{i}^{\top} b\right)+\lambda_{b}\|b\|_{1,2}
$$

where $\|b\|_{1,2}=\sum_{j=1}^{p} \sqrt{b_{j}^{2}+b_{j+p}^{2}}$ is the $\ell_{1,2}$ group norm that simultaneously shrinks the coefficients $b_{j}$ and $b_{j+p}, j=1, \ldots, p$, to zero. Consistency results for $\widehat{b}$ have not been established in the existing literature as it is challenging to deal with both the non-differentiable loss function and a nonparametric model. Analysis for this model is more challenging compared to the partially linear varying-coefficient model (Wang et al., 2009), where the nonparametric part is low-dimensional. Furthermore, the model in (1) is strictly more general than the partially linear varying-coefficient model.

\subsection{High dimensional inference}

We describe the challenges that arise in high-dimensional inference. Suppose first that we are interested in performing inference on a low-dimensional parameter $b^{\star} \in \mathbb{R}^{p}$, where the dimension $p$ is fixed as the sample size $n$ tends to infinity. After observing data, we can estimate $b^{\star}$ by minimizing some loss function $\mathcal{L}(b)=\mathcal{L}(b ;$ data $)$. For instance, in a regression problem with features $x_{i}$ and response $y_{i}, i=1, \ldots, n$, typically we would have $\mathcal{L}(b)=n^{-1} \sum_{i=1}^{n} \ell\left(b ; x_{i}, y_{i}\right)$, where $\ell(\cdot)$ is the negative log-likelihood under some assumed model.

In this classical setting, we can derive the well-known asymptotically normal distribution of the estimator $\widehat{b}$ around the true parameter value $b^{\star}$, by considering the score $\nabla \mathcal{L}(b)$. Namely, assuming that the loss is twice differentiable, by taking a Taylor expansion, we can see that the estimator $\widehat{b}$ satisfies

$$
0=\nabla \mathcal{L}(\widehat{b})=\nabla \mathcal{L}\left(b^{\star}\right)+\nabla^{2} \mathcal{L}\left(b^{\star}\right) \cdot\left(\widehat{b}-b^{\star}\right)+\Delta_{\text {Taylor }},
$$


where $\Delta_{\text {Taylor }}$ is the error in the Taylor expansion, equal to

$$
\Delta_{\text {Taylor }}=\left(\int_{0}^{1} \nabla^{2} \mathcal{L}\left((1-t) b^{\star}+t \widehat{b}\right) d t-\nabla^{2} \mathcal{L}\left(b^{\star}\right)\right) \cdot\left(\widehat{b}-b^{\star}\right) .
$$

Then by solving for $\widehat{b}$, we have

$$
\widehat{b}=b^{\star}+\left(-\nabla^{2} \mathcal{L}\left(b^{\star}\right)\right)^{-1} \cdot\left(\nabla \mathcal{L}\left(b^{\star}\right)+\Delta_{\text {Taylor }}\right) .
$$

Asymptotic normality of the error $\widehat{b}-b^{\star}$ then follows from two required properties: first, that the $\sqrt{n}$-score at the true parameter, $\sqrt{n} \nabla \mathcal{L}\left(b^{\star}\right)$, should be asymptotically normal via a central limit theorem argument, while the Taylor expansion error $\Delta_{\text {Taylor }}$ is vanishing at some appropriately fast rate; and second, that the term $\nabla^{2} \mathcal{L}\left(b^{\star}\right)$ should converge in probability to some fixed and invertible matrix (specifically, to its expectation).

In high dimensions, however, the above analysis fails. If $b \in \mathbb{R}^{p}$ where the dimension $p$ grows faster than the sample size $n$, then $\nabla^{2} \mathcal{L}\left(b^{\star}\right)$ will likely not converge in probability, and in general will not even be invertible. We can instead frame the argument in terms of a low-dimensional parameter of interest combined with a high-dimensional nuisance parameter. We write $b=\left(a^{\top}, c^{\top}\right)^{\top}$, where $a \in \mathbb{R}^{k}$ is the low-dimensional parameter of interests, while $c \in \mathbb{R}^{p-k}$ is the high-dimensional nuisance parameter. For example, if we are working in a regression model, where the loss takes the form $\mathcal{L}(b)=\sum_{i} \tilde{\ell}\left(y_{i} ; x_{i}^{\top} b\right)$ for some loss function $\tilde{\ell}$ (e.g., squared loss for a linear regression), then we might decompose the high-dimensional parameter vector as $b=\left(a^{\top}, c^{\top}\right)^{\top}$ to separate the coefficients on $k$ features of interest (without loss of generality, the first $k$ coordinates of the feature vectors $X_{i}$ ) and the remaining $p-k$ features, which we think of as potential confounders that need to be controlled for in the regression.

Suppose that our estimate of the low-dimensional parameter vector of interest, $a$, is obtained by solving

$$
\widehat{a}=\underset{a}{\arg \min } \mathcal{L}(a, \widetilde{c}),
$$

where $\widetilde{c}$ is some preliminary estimator of $c$. For example, in a high-dimensional regression problem, we may run an $\ell_{1}$-penalized regression first to obtain an initial sparse estimate of the parameters. Once an initial estimate is obtained, we can refit the low-dimensional vector $a$ without a penalty to remove the shrinkage bias. In this setting, we have

$$
\begin{array}{r}
0=\nabla_{a} \mathcal{L}(\widehat{a}, \widetilde{c})=\nabla_{a} \mathcal{L}\left(a^{\star}, c^{\star}\right)+\nabla_{a a}^{2} \mathcal{L}\left(a^{\star}, c^{\star}\right) \cdot\left(\widehat{a}-a^{\star}\right)+ \\
\nabla_{a c}^{2} \mathcal{L}\left(a^{\star}, c^{\star}\right) \cdot\left(\widetilde{c}-c^{\star}\right)+\Delta_{\text {Taylor2 }}
\end{array}
$$

where

$\Delta_{\text {Taylor2 }}$ 


$$
\begin{aligned}
& =\left(\int_{0}^{1} \nabla_{a a}^{2} \mathcal{L}\left((1-t) a^{\star}+t \widehat{a},(1-t) c^{\star}+t \widetilde{c}\right) d t-\nabla_{a a}^{2} \mathcal{L}\left(a^{\star}, c^{\star}\right)\right) \cdot\left(\widehat{a}-a^{\star}\right) \\
& +\left(\int_{0}^{1} \nabla_{a c}^{2} \mathcal{L}\left((1-t) a^{\star}+t \widehat{a},(1-t) c^{\star}+t \widetilde{c}\right) d t-\nabla_{a c}^{2} \mathcal{L}\left(a^{\star}, c^{\star}\right)\right) \cdot\left(\widetilde{c}-c^{\star}\right) .
\end{aligned}
$$

Therefore,

$\widehat{a}=a^{\star}+\left(-\nabla_{a a}^{2} \mathcal{L}\left(a^{\star}, c^{\star}\right)\right)^{-1}\left(\nabla_{a} \mathcal{L}\left(a^{\star}, c^{\star}\right)+\nabla_{a c}^{2} \mathcal{L}\left(a^{\star}, c^{\star}\right)\left(\widetilde{c}-c^{\star}\right)+\Delta_{\text {Taylor2 } 2}\right.$.

Let $S=\left(S_{a}^{\top}, S_{c}^{\top}\right)^{\top}=\left(\nabla_{a} \mathcal{L}^{\top}, \nabla_{c} \mathcal{L}^{\top}\right)^{\top}$ denote the score vector and the negative Hessian matrix is

$$
H=\left(\begin{array}{cc}
H_{a a} & H_{a c} \\
H_{c a} & H_{c c}
\end{array}\right)=-\left(\begin{array}{cc}
\nabla_{a a}^{2} \mathcal{L} & \nabla_{a c}^{2} \mathcal{L} \\
\nabla_{c a}^{2} \mathcal{L} & \nabla_{c c}^{2} \mathcal{L}
\end{array}\right)
$$

With this notation, we have

$$
\widehat{a}=a^{\star}+\left(H_{a a}\left(a^{\star}, c^{\star}\right)\right)^{-1} \cdot\left(S_{a}\left(a^{\star}, c^{\star}\right)-H_{a c}\left(a^{\star}, c^{\star}\right) \cdot\left(\widetilde{c}-c^{\star}\right)+\Delta_{\text {Taylor2 }}\right) .
$$

To assure the asymptotic normality of the error $\widehat{a}-a^{\star}$, we need to handle the following four terms:

- Asymptotic normality of $\sqrt{n} S_{a}\left(a^{\star}, c^{\star}\right)$, which will hold by a central limit theorem argument as before;

- Convergence in probability of $H_{a a}\left(a^{\star}, c^{\star}\right)$ to a fixed invertible matrix, which holds since $a \in \mathbb{R}^{k}$ is low-dimensional;

- Some control on the distribution of the term $H_{a c}\left(a^{\star}, c^{\star}\right) \cdot\left(\widetilde{c}-c^{\star}\right)$;

- Sufficiently small bound on $\Delta_{\text {Taylor2, which will hold as long as we assume }}$ that $(\widehat{a}, \widetilde{c})$ is sufficiently close to $\left(a^{\star}, c^{\star}\right)$.

The third term, $H_{a c}\left(a^{\star}, c^{\star}\right) \cdot\left(\widetilde{c}-c^{\star}\right)$, is the main challenge - since $c$ is highdimensional, in general it will not be possible to explicitly characterize the distribution of the error $\widetilde{c}-c^{\star}$ in its estimate. Therefore, we note that a naive refitting does not result in an asymptotically normal estimator and a different strategy is needed for high-dimensional inference.

One strategy to solve this problem is to modify the score method. Specifically, we want the term $H_{a c}\left(a^{\star}, c^{\star}\right) \cdot\left(\widetilde{c}-c^{\star}\right)$ to vanish at a sufficiently fast rate, so that it is smaller than the asymptotically normal term $S_{a}\left(a^{\star}, c^{\star}\right)$. The decorrelated score method, described next, provides such a result.

\subsection{The decorrelated score method}

When $\widehat{a}$ is defined as the minimizer of the objective function at some fixed estimator $\widetilde{c}$ for the nuisance parameter, $\widehat{a}=\arg \min _{a} \mathcal{L}(a, \widetilde{c})$, we can equivalently obtain $\widehat{a}$ as the solution to the score equation $0=\nabla_{a} \mathcal{L}(a, \widetilde{c})$. To decorrelate the score equations, we will instead define $\widehat{a}$ as the solution to $0=\nabla_{a} \mathcal{L}(a, \widetilde{c})-$ 
$V^{\top} \nabla_{c} \mathcal{L}(a, \widetilde{c})$, where $V \in \mathbb{R}^{(p-k) \times k}$ is a carefully chosen matrix, whose choice will be discusses in detail shortly. The Taylor expansion around the true parameter then gives us

$$
\begin{aligned}
0=S_{a}(\widehat{a}, \widetilde{c})-V^{\top} S_{c}(\widehat{a}, \widetilde{c})=S_{a}\left(a^{\star}, c^{\star}\right)-V^{\top} S_{c}\left(a^{\star}, c^{\star}\right) & -\left(H_{a a}\left(a^{\star}, c^{\star}\right)-V^{\top} H_{c a}\left(a^{\star}, c^{\star}\right)\right) \cdot\left(\widehat{a}-a^{\star}\right) \\
& \quad-\left(H_{a c}\left(a^{\star}, c^{\star}\right)-V^{\top} H_{c c}\left(a^{\star}, c^{\star}\right)\right) \cdot\left(\widetilde{c}-c^{\star}\right)+\text { Rem. }
\end{aligned}
$$

Solving for $\widehat{a}$, we then obtain

$$
\begin{aligned}
& \widehat{a}=a^{\star}+(\underbrace{H_{a a}\left(a^{\star}, c^{\star}\right)-V^{\top} H_{c a}\left(a^{\star}, c^{\star}\right)}_{\text {Term } 1})^{-1} . \\
&(\underbrace{\left(S_{a}\left(a^{\star}, c^{\star}\right)-V^{\top} S_{c}\left(a^{\star}, c^{\star}\right)\right)}_{\text {Term } 2}- \\
&\underbrace{\left(H_{a c}\left(a^{\star}, c^{\star}\right)-V^{\top} H_{c c}\left(a^{\star}, c^{\star}\right)\right) \cdot\left(\widetilde{c}-c^{\star}\right)}_{\text {Term } 3}+\operatorname{Rem}) .
\end{aligned}
$$

In order to show that $\widehat{a}$ is asymptotically normal, we would like to show that Term 1 converges in probability to a fixed (and invertible) matrix; Term 2 converges to a normal distribution via a central limit theorem argument; and Term 3 is vanishing (relative to Term 2). The role of the matrix $V$ is precisely to make Term 3 of smaller order compared to Term 2. Specifically, the matrix $V$ is chosen so that $\nabla_{a c}^{2} \mathcal{L}\left(a^{\star}, c^{\star}\right) \approx V^{\top} \nabla_{c c}^{2} \mathcal{L}\left(a^{\star}, c^{\star}\right)$, enabling us to show that Term 3 is vanishing without obtaining a limiting distribution for the high-dimensional estimator $\widetilde{c}$. In general, the matrix $V$ cannot be known in advance and is therefore data-dependent rather than fixed. However, in applications we will have that $V$ converges to some fixed matrix sufficiently fast and all the statements above still hold.

Finding the roots of the score equation may be numerically difficult. We present two methods that can be used in order to obtain $\widehat{a}$ that approximately satisfies the score equation next.

The first method is the one-step correction method. Define

$$
W=\left(\begin{array}{c}
I_{k} \\
-V
\end{array}\right) \cdot\left(H_{a a}\left(a^{\star}, c^{\star}\right)-V^{\top} H_{c a}\left(a^{\star}, c^{\star}\right)\right)^{-\top} .
$$

Expanding $W^{\top} S(\widetilde{a}, \widetilde{c})$ at $\left(a^{\star}, c^{\star}\right)$ and reorganizing the terms, we obtain

$$
\widetilde{a}+W^{\top} S(\widetilde{a}, \widetilde{c})=a^{\star}+W^{\top} S\left(a^{\star}, c^{\star}\right)-W^{\top} H_{\cdot c}\left(a^{\star}, c^{\star}\right) \cdot\left(\widetilde{c}-c^{\star}\right)+\operatorname{Rem},
$$

where $H_{\cdot c}=\left(\begin{array}{c}H_{a c} \\ H_{c c}\end{array}\right)$ and $\widetilde{b}=\left(\widetilde{a}^{\top}, \widetilde{c}^{\top}\right)^{\top}$ is a preliminary, consistent estimator of $b^{\star}$. Note that the form of the equation above is the same as in (8). This 
motivates us to define the one-step corrected estimator

$$
\check{a}^{O S}=\widetilde{a}+W^{\top} S(\widetilde{a}, \widetilde{c}) .
$$

Similar to the earlier discussion after (8), the normality of $\check{a}^{O S}$ will follow if we choose the matrix $W$ so that $W^{\top} H_{\cdot_{c}} \approx 0_{k, p-k}$ and $W$ itself converges to some fixed matrix sufficiently fast.

The second method for constructing $\widehat{a}$ relies on the reparametrization of the loss function. In the method sketched above, $\widehat{a}$ is defined as the minimizer of the objective function at a fixed preliminary estimate $\widetilde{c}$ of the nuisance parameter, i.e., $\widehat{a}=\arg \min _{a} \mathcal{L}(a, \widetilde{c})$. We saw above that the bottleneck in this analysis is the nonzero off-diagonal block of the Hessian matrix, $H_{a c}\left(a^{\star}, c^{\star}\right)$. To avoid the problematic term in the Taylor expansion, we can reparametrize the loss in such a way that the new off-diagonal block will become close to zero. Specifically, consider defining $\widehat{a}$ as the solution to a different optimization problem,

$$
\widehat{a}=\underset{a}{\arg \min } \mathcal{L}(a, \widetilde{c}-V(a-\widetilde{a})),
$$

where $\widetilde{a}, \widetilde{c}$ are preliminary estimates of $a^{\star}, c^{\star}$.

To better understand the approach in (9), consider again a regression setting where the distribution of each response variable $y_{i}$ is modeled as a function of $x_{i}^{\top} b=\left(x_{i, A}^{\top}, x_{i, A^{c}}^{\top}\right)\left(a^{\top}, c^{\top}\right)^{\top}$, where the subset $A \subset\{1, \ldots, p\}$ indexes the $k$ features of interest corresponding to the subvector $a$ of the regression coefficients. In this setting, the negative Hessian matrix $H_{a c}\left(a^{\star}, c^{\star}\right)$ will be nonzero whenever features in $A$ are correlated with features in $A^{c}$; thus, to set this block of the Hessian matrix to be (close to) zero, we can think of modifying the features of interest in the set $A$ by regressing out the confounding features in $A^{c}$. Specifically, let $v_{j} \in \mathbb{R}^{p-k}$ be the coefficient vector when regressing the feature $j \in A$ on all features in $A^{c}$. Then

$$
x_{i}^{\top} b=\left(x_{i, A}^{\top}, x_{i, A^{c}}^{\top}\right)\left(a^{\top}, c^{\top}\right)^{\top}=\left(x_{i, A}-V^{\top} x_{i, A^{c}}\right)^{\top} a+x_{i, A^{c}}^{\top}(c+V a),
$$

where $V \in \mathbb{R}^{(p-k) \times k}$ is the matrix with columns $v_{j}$. Note that, in this rearranged expression, the features of interest have been modified to be approximately orthogonal to, or approximately independent from, the nuisance features. Suppose we take $\widetilde{c}+V \widetilde{a}$ as the preliminary estimate of the coefficients $c+V a$ on the confounding features in this new model. If we then re-estimate the parameter vector of interest $a$, obtaining a new estimate $\widehat{a}$, then the final fitted regression is given by

$$
\left(x_{i, A}-V^{\top} x_{i, A^{c}}\right)^{\top} \widehat{a}+x_{i, A^{c}}^{\top}(\widetilde{c}+V \widetilde{a})=\left(x_{i, A}, x_{i, A^{c}}\right)^{\top}(\widehat{a}, \widetilde{c}-V(\widehat{a}-\widetilde{a})),
$$

thus motivating the form of the optimization problem given above in (9).

Defining $\widehat{a}$ as the solution to the decorrelated optimization problem (9), the Taylor expansion then gives us

$$
0=\left.\nabla_{a} \mathcal{L}(a, \widetilde{c}-V(a-\widetilde{a}))\right|_{a=\widehat{a}}
$$




$$
\begin{aligned}
& =S_{a}(\widehat{a}, \widetilde{c}-V(\widehat{a}-\widetilde{a}))-V^{\top} S_{c}(\widehat{a}, \widetilde{c}-V(\widehat{a}-\widetilde{a})) \\
& =S_{a}\left(a^{\star}, c^{\star}\right)-V^{\top} S_{c}\left(a^{\star}, c^{\star}\right)-\left(H_{a a}\left(a^{\star}, c^{\star}\right)-V^{\top} H_{c a}\left(a^{\star}, c^{\star}\right)\right) \cdot\left(\widehat{a}-a^{\star}\right) \\
& \quad-\left(H_{a c}\left(a^{\star}, c^{\star}\right)-V^{\top} H_{c c}\left(a^{\star}, c^{\star}\right)\right) \cdot\left(\widetilde{c}-V(\widehat{a}-\widetilde{a})-c^{\star}\right)+\operatorname{Rem},
\end{aligned}
$$

where Rem is redefined appropriately as the error term in this new expansion. Solving for $\widehat{a}$, we then obtain

$$
\begin{aligned}
\widehat{a}=a^{\star} & +(\underbrace{H_{a a}\left(a^{\star}, c^{\star}\right)-V^{\top} H_{c a}\left(a^{\star}, c^{\star}\right)}_{\text {Term } 1})^{-1} \cdot(\underbrace{\left(S_{a}\left(a^{\star}, c^{\star}\right)-V^{\top} S_{c}\left(a^{\star}, c^{\star}\right)\right)}_{\text {Term } 2} \\
& -\underbrace{\left(H_{a c}\left(a^{\star}, c^{\star}\right)-V^{\top} H_{c c}\left(a^{\star}, c^{\star}\right)\right) \cdot\left(\widetilde{c}-V(\widehat{a}-\widetilde{a})-c^{\star}\right)}_{\text {Term } 3}+\operatorname{Rem}) \cdot \quad(10)
\end{aligned}
$$

Therefore, $\widehat{a}$ is going to be asymptotically normal if Term 1 converges in probability to a fixed (and invertible) matrix; $\sqrt{n}$. Term 2 converges to a mean-zero normal distribution via a central limit theorem argument; and Term 3 and the remaining error Rem are vanishing (relative to Term 2). As before, the role of the matrix $V$ is in controlling Term 3. Specifically, the matrix $V$ is chosen so that $H_{a c}\left(a^{\star}, c^{\star}\right) \approx V^{\top} H_{c c}\left(a^{\star}, c^{\star}\right)$, enabling us to show that Term 3 is vanishing without obtaining a limiting distribution for the high-dimensional initial estimates $\widetilde{a}, \widetilde{c}$. In general, the matrix $V$ cannot be known in advance and is therefore data-dependent rather than fixed, but in our analysis we will see that as long as $V$ itself is sufficiently close to some fixed matrix, all the statements above will still hold.

\subsection{Non-differentiable loss in quantile regression}

When the loss function $\mathcal{L}$ is non-differentiable, which is the case in quantile regression, approaches based on the decorrelated score method cannot be directly applied. However, a simple modification allows us to proceed in a similar way as before. Assuming that the loss is nondifferentiable and convex, we let $S(a, c)$ denote the subdifferential of the loss. While $S(\cdot)$ might be highly nondifferentiable, its expected value is smooth in many problems. Therefore, we can compute the Hessian as the gradient of the expected value of $S(\cdot)$. In particular, we define the expected score function $\mathbf{E} S(a, c)$ as the expectation of the score $S(\cdot)$ at any fixed parameter choice $(a, c)$. Here it is important to note that, for a random parameter vector $(\widehat{a}, \widetilde{c})$, the expected score function $\mathbf{E} S(\widehat{a}, \widetilde{c})$ is not equal to $\mathbb{E}[S(\widehat{a}, \widetilde{c})]$, since this second quantity would evaluate its expectation with respect to the random values of $\widehat{a}$ and $\widetilde{c}$ as well. With the expected score function defined, we let

$$
H(a, c)=-\nabla \mathbf{E} S(a, c)
$$

be the negative gradient of the expected score. 
We specialize the discussion so for to the quantile regression problem at hand. We will base the inference procedures on the local linear formulation of the estimation problem for the varying-coefficient quantile regression model (3). Suppose $A=\{1, \ldots, k\}$ is the index set for the parameters of interest. Let $Y \in \mathbb{R}^{n}$ be the response and $U \in \mathbb{R}^{n}$ be the index for the varying coefficient. The matrix of input variables is denoted as $X=\left(X_{A}, X_{A^{c}}\right) \in \mathbb{R}^{n \times p}$, where $X_{A} \in \mathbb{R}^{n \times k}$ represents the features of interest and $X_{A^{c}} \in \mathbb{R}^{n \times(p-k)}$ the other features. Let

$$
\Gamma(u)=\left(X_{A}, X_{A^{c}}, \operatorname{diag}(U-u) X_{A}, \operatorname{diag}(U-u) X_{A^{c}}\right) \in \mathbb{R}^{n \times 2 p},
$$

and $\Gamma_{i}^{\top}(u)=\left(x_{i, A}^{\top}, x_{i, A^{c}}^{\top},\left(u_{i}-u\right) \cdot x_{i, A}^{\top},\left(u_{i}-u\right) \cdot x_{i, A^{c}}^{\top}\right)$ represents the $i$ th row vector of $\Gamma(u)$. The score function for quantile regression is given as

$$
\begin{aligned}
S\left(a_{0}, a_{1}, c_{0}, c_{1}\right)=\sum_{i \in[n]} w_{i} \cdot \Gamma_{i}(u) \cdot \Psi_{\tau} & \left(y_{i}-x_{i, A}^{\top} a_{0}-x_{i, A^{c}}^{\top} c_{0}\right. \\
& \left.-\left(u_{i}-u\right) \cdot x_{i, A}^{\top} a_{1}-\left(u_{i}-u\right) \cdot x_{i, A^{c}}^{\top} c_{1}\right),
\end{aligned}
$$

where $\Psi_{\tau}(u)=\tau-\mathbb{I}(u<0)$. Let $b_{0}=\left(a_{0}^{\top}, c_{0}^{\top}\right)^{\top} \in \mathbb{R}^{p}, b_{1}=\left(a_{1}^{\top}, c_{1}^{\top}\right)^{\top} \in \mathbb{R}^{p}$, and $b=\left(b_{0}^{\top}, b_{1}^{\top}\right)^{\top} \in \mathbb{R}^{2 p}$. Then the above score function can be written as

$$
S(b)=\sum_{i \in[n]} w_{i} \cdot \Gamma_{i}(u) \cdot \Psi_{\tau}\left(y_{i}-\Gamma_{i}^{\top}(u) \cdot b\right) .
$$

Let $b^{\star}=b^{\star}(\tau, u)$ be defined as a solution to $0=\mathbb{E}[S(b)]$ when $h \rightarrow 0$. Let $\tilde{q}_{i}(\tau, u)=\Gamma_{i}^{\top}(u) b^{\star}(\tau, u)$ be a local linear approximation to $q_{i}(\tau)=q\left(x_{i} ; \tau, u_{i}\right)$. Since $(\tau, u)$ is fixed, we write $\Gamma_{i}(u)=\Gamma_{i}, \tilde{q}_{i}(\tau, u)=\tilde{q}_{i}$ and $q_{i}(\tau)=q_{i}$ for notational simplicity. Finally, we use $\Delta_{i}=\Delta_{i}(\tau, u)=\tilde{q}_{i}-q_{i}$ to denote the approximation error from using the local linear model for the conditional quantile.

An approximate negative Hessian corresponding to the expected score function is given as

$$
H^{\star}=H\left(b^{\star} ; \tau, u\right)=\sum_{i \in[n]} w_{i} \cdot f_{i}\left(q_{i}+\Delta_{i}\right) \cdot \Gamma_{i} \Gamma_{i}^{\top} .
$$

Let $V^{\star} \in \mathbb{R}^{2 k \times 2 p}$ be the rows related to $X_{A}, X_{A}(U-u)$ of an approximate inverse of $H$ such that

$$
\left\|V^{\star} H^{\star}-E_{a}\right\|_{\infty, F} \leq \lambda^{\star}
$$

where

$$
\left\|V^{\star}\right\|_{\infty, F}=\sup _{i \in[k], j \in[p]}\left\|V_{(i, i+k),(j, j+p)}^{\star}\right\|_{F},
$$

$E_{a}=\left(e_{1}, \cdots, e_{2 k}\right)^{\top} \in \mathbb{R}^{2 k \times 2 p}$, and $\lambda^{\star}$ is a parameter that will be precisely given in Section 4.

With these preliminaries, we define the one-step correction estimator $\check{a}^{O S}$ as $\check{a}^{O S}=\widehat{a}-S_{d}(\widehat{b}, \widehat{V})$, where

$$
S_{d}(b, V):=\sum_{i \in[n]} S_{d i}(b, V)=\sum_{i \in[n]}-w_{i} V \Gamma_{i} \Psi_{\tau}\left(y_{i}-\Gamma_{i}^{\top} b\right),
$$

and $\widehat{V}, \widehat{b}$ are plug-in estimators of $V^{\star}, b^{\star}$ to be defined later, and $\widehat{a}=\widehat{b}_{1: 2 k}$. 


\section{Algorithm}

We provide computational details for the three proposed estimators. The first estimator is based on finding the root of the decorrelated-score; the second estimator is based on the one-step correction; and the third estimator is based on the reparametrization of the loss function. As discussed in the previous section, all these estimators are asymptotically equivalent. Estimation proceeds in three steps with the first two steps being the same for all three estimators. In the first step we obtain a pilot estimator of $b^{\star}$, while in the second step we obtain $\hat{V}$. We provide details next.

Step 1. Obtain the initial estimator $\widehat{b}^{\text {ini }}$ by minimizing the optimization program (3). The kernel weights are given as $w_{i}=(n h)^{-1} K\left(h^{-1} U_{i}-u\right)$, while the penalty parameter $\lambda_{b}$ is defined in a data dependent fashion as

$$
\lambda_{b}=c_{b} \sqrt{\tau(1-\tau) \log (n h p)} \cdot\left(\max _{j \in[p]} \mathbb{S}_{n}\left[w_{i}^{2} x_{i j}^{2}\right]\right)^{1 / 2}
$$

where $\mathbb{S}_{n}\left[z_{i}\right]$ denotes the summation, $\mathbb{S}_{n}\left[z_{i}\right]=\sum_{i \in[n]} z_{i}$, and $c_{b}$ is a data independent constant. We subsequently threshold elements of $\widehat{b}^{\text {ini }}$ to obtain $\widehat{b}$ with

$$
\widehat{b}_{j}= \begin{cases}\widehat{b}_{j}^{\text {ini }} \cdot \mathbb{1}\left\{\left(\widehat{b}_{j}^{\text {ini }}\right)^{2}+\left(\widehat{b}_{j+p}^{\text {ini }}\right)^{2}>\lambda_{b}^{2}\right\}, & j=1, \ldots, p, \\ \widehat{b}_{j}^{\text {ini }} \cdot \mathbb{1}\left\{\left(\widehat{b}_{j}^{\text {ini2 } 2}\right)^{2}+\left(\widehat{b}_{j-p}^{\text {ini }}\right)^{2}>\lambda_{b}^{2}\right\}, & j=p+1, \ldots, 2 p,\end{cases}
$$

to ensure the sparsity of the estimator for Theorem 1.

Step 2. Obtain $\widehat{V}$ by

$$
\widehat{V}=\underset{V \in \mathbb{R}^{2 k \times 2 p}}{\arg \min }\left\{\operatorname{trace}\left(\frac{1}{2} V \widehat{H} V^{\top}-E_{a} V^{\top}\right)+\lambda_{V}\|V\|_{1, F}\right\},
$$

where $\lambda_{V}=n^{-1} c_{v} \sqrt{n h} \Phi^{-1}\left(1-\frac{0.05}{2 n h p}\right)$ with $c_{v}$ being a data independent constant; and $\widehat{H}=\sum_{i \in[n]} w_{i} \hat{f}_{i} \Gamma_{i} \Gamma_{i}^{\top}$ with $w_{i}$ being the kernel weight as defined in Step 1 and $\hat{f}_{i}$ is computed with a data adaptive procedure as

$$
\hat{f}_{i}=\frac{\mathbb{1}\left\{\left|y_{i}-\Gamma_{i}^{\top} \widehat{b}\right| \leq h_{f}\right\}}{2 h_{f}}
$$

with

$$
h_{f}=\left(\Phi^{-1}\left(\tau+h_{p}\right)-\Phi^{-1}\left(\tau-h_{p}\right)\right) \min \left\{\sqrt{\operatorname{Var}(\hat{e})}, \frac{Q_{0.75}(\hat{e})-Q_{0.25}(\hat{e})}{1.34}\right\},
$$

where

$$
\hat{e}_{i}=y_{i}-\Gamma_{i}^{\top} \widehat{b}, \quad Q_{\alpha}(\hat{e})=\inf _{q}\left\{q: \frac{\sum_{i} w_{i} \mathbb{1}\left\{\hat{e}_{i} \leq q\right\}}{\sum_{i} w_{i}} \geq \alpha\right\}
$$




$$
\operatorname{Var}(\hat{e})=\frac{\sum_{i} w_{i}\left(\hat{e}_{i}-\frac{\sum_{j} w_{j} \hat{e}_{j}}{\sum_{j} w_{j}}\right)^{2}}{\sum_{i} w_{i}}
$$

and

$$
h_{p}=n^{-1 / 3}\left\{\Phi^{-1}(0.975)\right\}^{2 / 3}\left\{\frac{1.5\left[\phi\left(\Phi^{-1}(\tau)\right)\right]^{2}}{2\left[\Phi^{-1}(\tau)\right]^{2}+1}\right\}^{1 / 3}
$$

is the Powell bandwidth defined in Koenker (2005, Section 3.4.4).

Step 3. Here we obtain our final estimator $\check{a}$ using one of the three procedures.

1. Finding the root of the decorrelated score. We would like to construct $\check{a}$ by solving for $\sum_{i} \widetilde{S}_{i}(a)=0$ where $\widetilde{S}_{i}(a)=S_{d i}\left(\left(a^{\top}, \widehat{c}^{\top}\right)^{\top}, \widehat{V}\right)$ with $S_{d i}$ defined in (13). Because $\sum_{i} \widetilde{S}_{i}(a)$ is not continuous, we can approximately solve the equation by

$$
\check{a}^{D S}=\arg \min \left[\sum_{i} \widetilde{S}_{i}(a)\right]^{\top}\left[\sum_{i} \widetilde{S}_{i}(a) \widetilde{S}_{i}^{\top}(a)\right]^{-1}\left[\sum_{i} \widetilde{S}_{i}(a)\right] .
$$

Minimizing the above problem is not computationally simple. The following two strategies might be preferred, as discussed in Section 2.3.

2. The one step correction estimator. We compute the estimator as

$$
\check{a}^{O S}=\widehat{a}+\sum_{i \in[n]} w_{i} \widehat{V} \Gamma_{i}^{\top} \Psi_{\tau}\left(y_{i}-\Gamma_{i}^{\top} \widehat{b}\right) .
$$

3. The reparametrization estimator. We first obtain $\tilde{\Gamma}_{i}, \tilde{y}_{i}$ as $\tilde{\Gamma}_{i}=\Gamma_{i, A}-$ $\widehat{V}_{2} \Gamma_{i, A^{c}}$ and $\tilde{y}_{i}=y_{i}-\Gamma_{i, A^{c}}^{\top}\left(\widehat{c}+\widehat{V}_{2}^{\top} \widehat{a}\right)$, where $\widehat{V}_{2}=\widehat{V}_{11}^{-1} \widehat{V}_{12}, \widehat{V}_{11}=\widehat{V}_{1: 2 k} \in$ $\mathbb{R}^{2 k \times 2 k}$ and $\widehat{V}_{12}=\widehat{V}_{(2 k+1): 2 p} \in \mathbb{R}^{2 k \times 2(p-k)}$. Then the estimators is computed as

$$
\check{a}^{R P}=\underset{a}{\arg \min } \sum_{i} w_{i} \cdot \rho_{\tau}\left(\tilde{y}_{i}-\tilde{\Gamma}_{i}^{\top} a\right) .
$$

With the estimator $\check{a}$, being $\check{a}^{D S}, \check{a}^{O S}$, or $\check{a}^{P R}$, we can perform statistical inference about the parameter of interests, $a$. For any one of the three estimamors, we have that

$$
\widehat{\Sigma}_{a}^{-1 / 2}(\check{a}-a) \sim \mathcal{N}\left(\mathbf{0}, I_{k}\right),
$$

where the covariance matrix is computed as

$$
\widehat{\Sigma}_{a}=n h \sum_{i} w_{i}^{2} \widehat{V} \Gamma_{i}^{\top} \Psi_{\tau}^{2}\left(y_{i}-\Gamma_{i}^{\top} \widehat{b}\right) \Gamma_{i} \widehat{V}^{\top} .
$$

We end this section with some remarks on the computation. The kernel weights we chose in our simulation studies are given as

$$
w_{i}=(n h)^{-1} \mathbb{1}\left\{\left|U_{i}-u\right| / h<0.5\right\}
$$

with $h=c_{h} n^{-1 / 3}$ and $c_{h}=4$. However, we note that any kernel function that satisfies Assumption 1 presented later in Section 4.1 can be used. Many frequently used kernels, such as the Gaussian kernel and box kernel, satisfy this 
assumption. We also set $c_{b}=0.4$ and $c_{v}=0.02$ in our numerical studies. The performance of the algorithm is not very sensitive to the choice of these parameters. Both in Step 1 and Step 2 of the algorithm, one can perform optional refitting of the selected coefficients to improve finite sample performance. For example, in Step 1, let $S \in \mathbb{R}^{s}$ be the support of the covariates in $\Gamma$ corresponding to $b$, and $\Gamma_{i, S}$ and $b_{S}$ are the corresponding entries in $\Gamma_{i}$ and $b$, then

$$
\widehat{b}_{\text {post }}=\left\{\underset{b \in \mathbb{R}^{p}}{\arg \min } \sum_{i=1}^{n} w_{i} \cdot \rho_{\tau}\left(y_{i}-\Gamma_{i, S}^{\top} b_{S}\right): b_{j}=0 \forall j \in S^{c}\right\}
$$

can be used to replace $\widehat{b}$.

\section{Main results}

In this section, we present our main results. We start by detailing the assumptions in Section 4.1. Results on estimation consistency are presented in Section 4.2. Finally, we give results on the asymptotic normality of the estimator in Section 4.3.

\subsection{Assumptions}

We state the assumptions needed to establish our results.

Assumption 1 (Kernel assumptions). The kernel function $K(\cdot)$ satisfies

$$
\begin{gathered}
K(t) \leq \nu_{0}<\infty \text { for all }, \quad \int K(u) d u=\nu_{1}<\infty, \\
\int K^{2}(u) d u=\nu_{2}<\infty, \quad \int K(u) u^{2} d u=\mu_{2}<\infty, \\
\int K(u) u^{4} d u=\mu_{4}<\infty .
\end{gathered}
$$

The kernel is chosen by a statistician, so the above assumption does not put restrictions on the data generating process. A number of standard kernels such as the Gaussian kernel, box kernel, and Epanechnikov kernel, all satisfy the above assumption.

Assumption 2 (Assumptions on $U$ ). We assume $U$ has bounded support. Without loss of generality, we assume $U \in[0,1]$. Let $f_{U}(u)$ be the density of $U$. There exists $\bar{f}$ such that $f_{U}(u) \leq \bar{f}$.

From Assumptions 1 and 2, the kernel weights $w_{i}$ 's satisfy the following with high probability

$$
\left\|w_{i}\right\|_{\infty} \leq B_{w}=\frac{\nu_{0}}{n h} \leq \frac{B_{K}}{n h}, \quad \sum_{i} w_{i} \leq B_{K}
$$




$$
\sum_{i} w_{i}\left(\frac{u_{i}-u}{h}\right)^{2} \leq B_{K} \text {, and } \sum_{i} w_{i}\left(\frac{u_{i}-u}{h}\right)^{4} \leq B_{K}
$$

for some constant $B_{K}>0$.

Assumption 3 (Assumptions on the distribution of $Y$ ). Let $f_{i}(y)$ be the conditional density of $Y_{i}$ given $X_{i}=x_{i}, U_{i}=u_{i}$. We assume that there exist constants $\underline{f}, \bar{f}, \bar{f}^{\prime}$ such that

$$
0<\underline{f} \leq f_{i}(y) \leq \bar{f}, \quad \text { and } \quad\left|f_{i}^{\prime}(y)\right| \leq \bar{f}^{\prime} \quad \text { for all } y
$$

This type of assumption on the conditional distribution of $Y$ is commonly used in the literature on quantile regression, for example, see Belloni et al. (2016a).

Assumption 4 (Approximate linear sparsity and smoothness of $q(x ; \tau, u)$ ). Assume there exists a smooth and sparse $\beta^{\star}(\tau, u)$ such that:

- $u \mapsto \beta^{\star}(\tau, u)$ is differentiable for all $\tau \in[\varepsilon, 1-\varepsilon]$ and

$$
\left\|\beta^{\star}\left(\tau, u^{\prime}\right)-\beta^{\star}(\tau, u)-\left(u^{\prime}-u\right) \cdot \nabla_{u} \beta^{\star}(\tau, u)\right\|_{2} \leq B_{\beta}\left(u^{\prime}-u\right)^{2} ;
$$

- the supports of $\beta^{\star}(u, \tau)$ and $\partial_{u} \beta^{\star}(\tau, u)$ are sparse; that is, for the sets

$$
S:=\left\{j \in[p] \mid \beta_{j}^{\star}(\tau, u) \neq 0\right\}
$$

and

$$
S^{\prime}:=\left\{j \in[p] \mid \beta_{j}^{\star}(\tau, u) \neq 0 \text { or } \partial_{u} \beta_{j}^{\star}(\tau, u) \neq 0\right\},
$$

we have $s:=|S| \ll n$ and $\left|S^{\prime}\right| \leq s_{1}:=c_{1} s$ for some constant $c_{1}$.

We assume that the quantile function $q_{i}=q\left(x_{i} ; \tau, u_{i}\right)$ can be well approximated by a linear function $x_{i}^{\top} \beta^{\star}\left(\tau, u_{i}\right)$; specifically,

$$
\mathbb{P}\left\{Y \leq x_{i}^{\top} \beta^{\star}\left(\tau, u_{i}\right) \mid X=x_{i}, U=u_{i}\right\}=\tau+R_{i},
$$

where

$$
\sqrt{\sum_{i} w_{i} R_{i}^{2}}=\epsilon_{R}=O\left(\sqrt{\frac{\log (n p)}{n h}}\right) .
$$

This assumption requires that the conditional quantiles of $Y$ approximately follow a linear varying-coefficient model and the approximation error is vanishing as $n \rightarrow \infty$. In addition, the varying-time coefficient $\beta^{\star}(\tau, u)$ is Hölder smooth, sparse and has sparse first derivatives.

For the case when $u$ and $\tau$ are fixed, we will write $\beta^{\star}=\beta^{\star}(\tau, u)$. Let $b^{\star}=$ $b^{\star}(\tau, u)=\left(\beta^{\star}(\tau, u)^{\top}, \nabla_{u}^{\top} \beta^{\star}(\tau, u)\right)^{\top}$ and write $q_{i}=q\left(x_{i} ; \tau, u_{i}\right), \tilde{q}_{i}=\Gamma_{i}^{\top} b^{\star}$ as a local linear approximation to $q_{i}$. Let

$$
H=\sum_{i \in[n]} w_{i} \cdot f_{i}\left(q_{i}\right) \cdot \Gamma_{i} \Gamma_{i}^{\top} \text { and } H^{\star}=\sum_{i \in[n]} w_{i} \cdot f_{i}\left(\tilde{q}_{i}\right) \cdot \Gamma_{i} \Gamma_{i}^{\top} .
$$


Assumption 5 (Assumptions on the Hessian). Let $V^{\star}$ be the rows related to $\left[X_{A}, X_{A}(U-u)\right]$ of an approximate inverse of $H^{\star}$. We assume that

$$
\left\|H^{\star} V^{\star}-E_{a}\right\|_{\infty, F} \leq \lambda^{\star}=O\left(B \sqrt{\frac{\log p}{n h h_{f}}}\right), \quad\left\|V^{\star}\right\|_{0, F} \leq s_{2}=c_{2} s,
$$

where $\|V\|_{0, F}:=\left|\left\{(i, j): i \in[k], j \in[p], V_{(i, i+k),(j, j+p)}^{\star} \neq 0\right\}\right|,\|V\|_{\infty, F}:=$ $\max _{i \in[k], j \in[p]}\left\|V_{(i, i+k),(j, j+p)}^{\star}\right\|_{F}$ and $\max _{i \in[n]}\left\|V^{\star} \Gamma_{i}\right\|_{2}=B_{V} \asymp \log p$. Furthermore, we have

$$
(n h)^{-1} s B_{V}^{2} \log p=o(1) \text { and } \log \left(B_{V}^{2} h_{f} h\right)=o(\log p) .
$$

Assumption 5 holds when $X_{A}$ follows a multivariate approximately sparse linear model with respect to $X_{A^{c}}$, where we require the coefficients to be approximately linear, sparse and smooth (see Appendix C). For example, when the distribution of $X$ does not depend on $U$ and the exact sparse linear model holds, Assumption 5 obviously holds.

Assumption 6 (Assumptions on $\mathrm{X}$ ). We make the following assumptions on the covariate $X$ :

- Boundedness: there exists a constant $B_{X}$ such that with high probability,

$$
\max _{i}\left\|x_{i}\right\|_{\infty} \leq B_{X} \text { and } \max _{j \in[p]} \sum_{i} w_{i}^{2} x_{i j}^{2} \leq \frac{B_{X}^{2} B_{K}^{2}}{n h} .
$$

- Restricted eigenvalues: Consider the following cones

$$
\begin{aligned}
\mathbb{C}\left(s_{1}\right) & =\left\{\theta:\|\theta\|_{0} \leq s_{1} \text { and }\|\theta\|_{2}=1\right\}, \text { and } \\
\mathbb{C}\left(S_{2}\right) & =\left\{\Theta \in \mathbb{R}^{2 k \times 2 p}:\left\|\Theta_{S_{2}^{c}}\right\|_{1, F} \leq 6\left\|\Theta_{S_{2}}\right\|_{1, F} \text { and }\|\Theta\|_{1, F}=1\right\},
\end{aligned}
$$

where $S_{2}=\left\{(i, j):\left\|V_{(i, i+k),(j, j+p)}^{\star}\right\|_{F}>0\right\}$ is the support of $V^{\star}$ By Assumption $5,\left|S_{2}\right| \leq s_{2}$. We assume there exist $0<\kappa_{-}^{2} \leq \kappa_{+}^{2}<\infty$ such that

$$
\begin{aligned}
& \kappa_{-}^{2} \leq \sum_{i \in[n]} w_{i}\left(\Gamma_{i}^{\top} \theta\right)^{2} \leq \kappa_{+}^{2} \quad \text { for all } \theta \in \mathbb{C}\left(s_{1}\right) \text { and } \\
& \kappa_{-}^{2} \leq \sum_{i \in[n]} w_{i} \operatorname{trace}\left(\Theta^{\top} \Gamma_{i} \Gamma_{i}^{\top} \Theta\right) \leq \kappa_{+}^{2} \quad \text { for all } \Theta \in \mathbb{C}\left(S_{2}\right) .
\end{aligned}
$$

- For some constant $\kappa_{q}>0$,

$$
\inf _{\substack{\|\delta\|_{1,2} \leq \frac{7\left|S^{\prime}\right| \cdot \sqrt{\log p}}{\kappa-\sqrt{n h}} \\ \mathbb{S}_{n}\left[w_{i}\left(\Gamma_{i}^{\top} \delta\right)^{2}\right]=\frac{\left|S^{\prime}\right| \log p}{n h}}} \frac{\left(\underline{f} \cdot \sum_{i \in[n]} w_{i} \cdot\left(\Gamma_{i}^{\top} \delta\right)^{2}\right)^{3 / 2}}{\bar{f}^{\prime} \cdot \sum_{i \in[n]} w_{i} \cdot\left(\Gamma_{i}^{\top} \delta\right)^{3}} \geq \kappa_{q} .
$$


The assumptions on the design $X$ are mild and commonly used in the literature on high-dimensional estimation and inference. For example, boundedness and restricted eigenvalue condition was used in Negahban et al. (2012). The condition (21) is a mild growth condition, which is satisfied for many design matrices $X$, see Belloni et al. (2016a) and Belloni and Chernozhukov (2011).

Finally, we need the following growth condition.

Assumption 7 (Growth conditions). We assume

$$
h \asymp O\left(n^{-1 / 3}\right), \quad h_{f} \asymp O\left(n^{-1 / 3}\right), \quad \text { and } \quad\left(n h h_{f}\right)^{-1 / 2} s \log p \log (n p)=o(1) .
$$

With these assumptions, we are ready to present our main results next.

\subsection{Consistency and sparsity results of the initial estimators}

We establish the asymptotic properties of the initial estimators in Step 1 and Step 2 from Section 3.

Theorem 1. Under Assumptions 1, 2, 3, 4, 6 and \%, the estimator $\widehat{b}$ from Step 1 in Section 3 satisfies

$$
\begin{aligned}
\mathbb{S}_{n}\left[w_{i} \cdot\left(\Gamma_{i}^{\top}\left(\widehat{b}-b^{\star}\right)\right)^{2}\right] & \leq O_{p}\left(\frac{s \log (n p)}{n h}\right) \\
\left\|\widehat{b}-b^{\star}\right\|_{1,2} & \leq O_{p}\left(s \sqrt{\frac{\log (n p)}{n h}}\right), \text { and } \\
\|\widehat{b}\|_{0,2} & \leq O_{p}(s)
\end{aligned}
$$

where $\mathbb{S}_{n}\left[z_{i}\right]:=\sum_{i \in[n]} z_{i},\|b\|_{1,2}:=\sum_{i=1}^{p} \sqrt{b_{i}^{2}+b_{i+p}^{2}}$, and $\|b\|_{0,2}$ is defined as the $\ell_{0}$ norm of the vector $\left(\sqrt{b_{1}^{2}+b_{1+p}^{2}}, \cdots, \sqrt{b_{p}^{2}+b_{2 p}^{2}}\right)$.

Theorem 1 gives us convergence results regarding the $\ell_{1,2}$-penalized quantile regression estimator from (3). In particular, (22) gives the rate of convergence rate of the prediction $\ell_{2}$-norm, (23) gives the $\ell_{1,2}$-norm of the error, and (24) gives the sparsity of $\widehat{b}$ in Step 1 of Section 3. Both Theorem 2 and Theorem 3 rely on these conditions. The extra growth condition in Assumption 6 is mild. Specifically, with the penalty parameter $\lambda_{b} \asymp O\left(\sqrt{\frac{\log p}{n h}}\right)$ the assumption is satisfied. The sparsity here is achieved by truncating the small components in $\widehat{b}^{\text {ini }}$ to zero, while maintaining the same rate of convergence.

Theorem 2. Suppose the assumptions for Theorem 1 hold and the estimator $\widehat{b}$ obtained in Step 1 satisfies (22), (23), and (24). Furthermore, suppose that $\lambda_{V} \geq 2 \lambda^{\star}$ and Assumption 5 holds for $\lambda^{\star}$. Then $\widehat{V}$ from Step 2 satisfies

$$
\left\|\widehat{V}-V^{\star}\right\|_{F} \leq O_{p}\left(B_{V} \sqrt{\frac{s \log (n p)}{n h h_{f}}}\right) \quad \text { and }
$$




$$
\left\|\widehat{V}-V^{\star}\right\|_{1, F} \leq O_{p}\left(s B_{V} \sqrt{\frac{\log (n p)}{n h h_{f}}}\right),
$$

where $\|V\|_{1, F}:=\sum_{i \in[k], j \in[p]}\left\|V_{(i, i+k),(j, j+p)}\right\|_{F}$.

Theorem 2 gives the convergence rate of the $\ell_{1,2}$-norm and prediction $\ell_{2}$-norm of $\widehat{V}$ in Step 2 of Section 3. Because the $\widehat{H}$ in the objective function relies on the estimator $\widehat{b}$, both the convergence and sparsity results from Theorem 1 are needed.

\subsection{Normality result of the final estimators}

We state the asymptotic normality result for the one step estimator.

Theorem 3 (Normality for the one-step estimator). Assume that Assumptions 1 - 7 hold and (22), (23), (24), (25), (26) hold. Then the one step estimator defined in (17) satisfies

$$
\sqrt{n h} \widehat{\Sigma}_{a}^{-1 / 2}\left(\check{a}^{O S}-a^{\star}\right) \rightarrow_{d} \mathcal{N}\left(0, I_{2 k}\right)
$$

where the covariance matrix $\widehat{\Sigma}_{a}$ is estimated as either

$$
\widehat{\Sigma}_{a}=n h \widehat{V}\left\{\sum_{i} w_{i}^{2} \Gamma_{i} \Psi_{\tau}\left(y_{i}-\Gamma_{i}^{\top} \widehat{b}\right) \Psi_{\tau}\left(y_{i}-\Gamma_{i}^{\top} \widehat{b}\right)^{\top} \Gamma_{i}^{\top}\right\} \widehat{V}^{\top}
$$

or

$$
\widehat{\Sigma}_{a}=\tau(1-\tau) \nu_{2} \widehat{V}\left\{\sum_{j} w_{j} \Gamma_{j} \Gamma_{j}^{\top}\right\} \widehat{V}^{\top}
$$

Theorem 3 tells us that the one step estimator is $\sqrt{n h}$-consistent. The covariance (28) is the expected version of (27), where (27) comes from the central limit theorem. The estimators from the decorrelated score $\left(\check{a}^{D S}\right)$ and reparameterization $\left(\check{a}^{R P}\right)$ are both asymptotically equivalent to $\check{a}^{O S}$; the detailed proof is in the appendix.

\section{Numerical studies}

Through an empirical study, we investigate the finite sample performance of our confidence interval construction approach and show that it works under high-dimensional settings and is robust to different error distributions.

For each individual, the data is generated independently and identically distributed from the following distribution of $\left\{U, X_{1}, X_{-1}, \epsilon, Y\right\}$. First, we generate the index variables as $U \sim \operatorname{Unif}[0,2]$ and the confounding variables as

$$
X_{-1} \mid U \sim \mathcal{N}(\mu(U), \Sigma(U))
$$


where $\mu_{j}(U)=a_{0} \cdot j \cdot\left(U^{a_{1}}-1\right)$ and $\Sigma(U)$ is an autoregressive (AR) covariance with elements $\Sigma(U)_{i, j}=\rho(U)^{|i-j|}$ and the parameter $\rho(U)=\rho^{1+b_{0}\left(U^{b_{1}}-1\right)}$. Note that when $a_{1}=b_{1}=0$ we have a model where the nuisance covariates $X_{-1} \mid U$ are homogeneous and do not depend on the index variable $U$. We then generate $X_{1}$ and $Y$. Let $\nu \in \mathbb{R}^{p-1}$ with $\nu_{j-1}=1 / j^{2}, j=2, \cdots, p$, and $\beta=\left(\frac{1}{2}, c_{y} \nu^{\top}\right)^{\top}$. Then

$$
\begin{aligned}
& X_{1}=X_{-1}\left(c_{x} \nu\right)+\epsilon_{x}, \text { where } \epsilon_{x} \sim \mathcal{N}(0,1) \text { and is independent of }\left(X_{-1}, U\right), \\
& Y=X \beta(U)+\epsilon, \quad \text { where } \beta(U)=\beta\left(c_{0} U^{c_{1}}+1-c_{0}\right) \text {, } \\
& \text { and } \epsilon \mid X, U \sim \sigma_{e}(U) \cdot F_{e} \cdot \sqrt{\left(2-\gamma+\gamma \cdot X_{1}^{2}\right) / 2} \text {. }
\end{aligned}
$$

Note that $\epsilon$ is allowed to depend on $X$. In particular, $\gamma=0$ leads to a homogeneous setting and $\gamma=1$ leads to a heterogeneous setting. Here $\sigma_{e}(U)=$ $\sigma_{e}\left(1+d_{0}\left(U^{d_{1}}-1\right)\right)$ and $F_{e}$ is either the standard Gaussian or $t$ distribution with 3 degrees of freedom $(t(3))$.

Specially, this data generation process leads to the following quantiles:

$$
q(x ; \tau, u)=x \beta(u)+\sigma_{e}(u) \cdot \sqrt{\left(2-\gamma+\gamma \cdot x_{1}^{2}\right) / 2} \cdot q_{e}(\tau),
$$

where $q_{e}(\tau)$ is the $\tau$-th quantile of the distribution $F_{e}$. In this simulation, we are interested in the inference for $\beta_{1}(\tau, u) \in \mathbb{R}^{1}$ at the point $(\tau, u)=(0.5,1)$. At this point, $q(x ; 0.5,1)=x \beta(1)$ satisfy Assumption 5 .

The coefficients $c_{x}$ and $c_{y}$ are used to control the $R^{2}$ in different regression equations. We use $R_{y}^{2}$ to denote the $R^{2}$ in the equation $Y-X_{1} \beta_{1}(U)=$ $X_{-1} \beta_{-1}(U)+\epsilon$, while $R_{x}^{2}$ denotes the $R^{2}$ in the equation $X_{1}=X_{-1}\left(c_{x} \nu\right)+\epsilon_{x}$. We vary the parameters and choose $c_{y}, c_{x}$ to form different combinations of $\left(R_{y}^{2}, R_{x}^{2}\right)$. Details can be found in Appendix B.

We evaluate the performance of our algorithms described in Section 3 (DS (16), OS (17) and RP (18)) and compare them with the Oracle and the Naive methods. For the oracle method, we assume that the true (low dimensional) set of predictors is known in advance and our inference is based on the kernel weighted quantile regression on the true set of variables. For the Naive method, we fit the kernel weighted penalized regression as in Step 1. Then we fit the postregularized regression and do the inference treating the set $\hat{S}=\left\{j: \hat{\beta}_{j} \neq 0\right\}$ as fixed. We compare their performance from $M=100$ simulations in terms of the bias, empirical standard deviation (SD), the expected estimated standard error (ESE), and coverage rate for the 95\% nominal confidence intervals (CR).

The simulation results for two settings with normally distributed and $t(3)$ distributed $\epsilon$ 's are listed in Table 1. Additional simulation results are presented in Table 2 in Appendix B. From the simulation, the oracle method consistently produce confidence intervals with coverage rate close to the nominal value $95 \%$ in all simulation settings. The Naive estimator has some significant bias when $R_{y}^{2}$ is small and $R_{x}^{2}$ is large. Furthermore, without any correction, the confidence intervals tend to have significantly lower coverage than the nominal value. The OS, DS, and PR estimators have relatively low bias compared to the naive 
TABLE 1

Simulation results for the correlation setting $\left(R_{x}^{2}, R_{y}^{2}\right)=(0.7,0.3)$.

\begin{tabular}{c|cccccc}
\hline$\epsilon$ distribution & $\gamma$ & Method & Bias & SD & ESE & CR \\
\hline \multirow{5}{*}{ Normal } & & One Step & 0.022 & 0.063 & 0.076 & 0.97 \\
& & Decorrelated score & 0.057 & 0.139 & 0.124 & 0.92 \\
& 0 & Reparameterization & 0.045 & 0.055 & 0.076 & 0.97 \\
& & Naive & 0.274 & 0.047 & 0.045 & 0.41 \\
& & Oracle & -0.004 & 0.062 & 0.067 & 0.95 \\
\cline { 2 - 7 } & & One Step & 0.093 & 0.149 & 0.190 & 0.96 \\
& & Decorrelated score & 0.174 & 0.134 & 0.297 & 0.99 \\
& \multirow{4}{*}{1} & Reparameterization & 0.148 & 0.142 & 0.190 & 0.97 \\
& & Naive & 0.246 & 0.120 & 0.150 & 0.62 \\
& & Oracle & -0.034 & 0.133 & 0.140 & 0.94 \\
\hline \multirow{5}{*}{$t(3)$} & & One Step & 0.022 & 0.083 & 0.084 & 0.96 \\
& & Decorrelated score & 0.077 & 0.112 & 0.145 & 0.95 \\
& \multirow{4}{*}{0} & Reparameterization & 0.054 & 0.071 & 0.084 & 0.93 \\
& & Naive & 0.249 & 0.075 & 0.057 & 0.10 \\
& & Oracle & -0.020 & 0.042 & 0.051 & 0.95 \\
\cline { 2 - 7 } & & One Step & 0.090 & 0.193 & 0.212 & 0.95 \\
& \multirow{4}{*}{1} & Decorrelated score & 0.185 & 0.163 & 0.313 & 0.98 \\
& & Reparameterization & 0.167 & 0.173 & 0.212 & 0.94 \\
& & Naive & 0.234 & 0.156 & 0.186 & 0.73 \\
& & Oracle & -0.01 & 0.126 & 0.164 & 0.98 \\
\hline
\end{tabular}

method in all settings; also, their coverage rates are closer to the nominal value than the naive method.

We plot the trend of coverage rate for all methods with the change of $R_{y}^{2}$ and $R_{x}^{2}$ in Figure 1 to better understand the performance of the different methods as the data generating distribution changes. We find that the confidence intervals from the Naive method significantly undercover when the response $Y$ has low correlation with the covariates $X$ and $X_{1}$ has high correlation with the confounding variables $X_{-1}$. On the other hand, the proposed methods provide satisfactory coverage across all settings.

Regarding the widths of the confidence intervals, the Naive method underestimates the standard error in some data settings, resulting in low coverage rates. The OS, DS, and PR methods provide CI's with the correct coverage rate and the widths of the CI's are slightly larger than those of the Oracle method. Among the three proposed methods, the OS method has the best finite sample performance in terms of stability and computational cost.

\section{Real data example}

As an illustration of our method, we apply our methods to analyze the plasma beta-carotene level data set collected by a cross-sectional study (Nierenberg et al., 1989). This dataset consists of 315 observations on 14 variables. Our interest is to study the relationship between the plasma beta-carotene level and the following variables: age, sex, smoking status, quetelet (BMI), vitamin use, number of calories consumed per day, grams of fiber consumed per day, number 

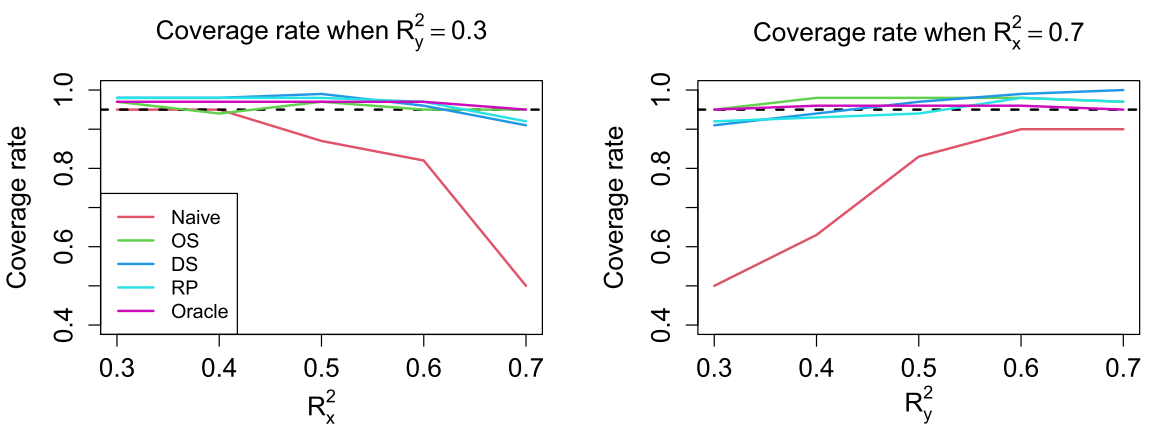

FIG 1. Left: $C R$ of different methods with $R_{y}^{2}$ fixed at 0.3 and changing $R_{x}^{2}$. Right: $C R$ of different methods with $R_{x}^{2}$ fixed at 0.7 and changing $R_{y}^{2}$. In the simulation, the error term $\epsilon$ is normally distributed and $\gamma=0$.
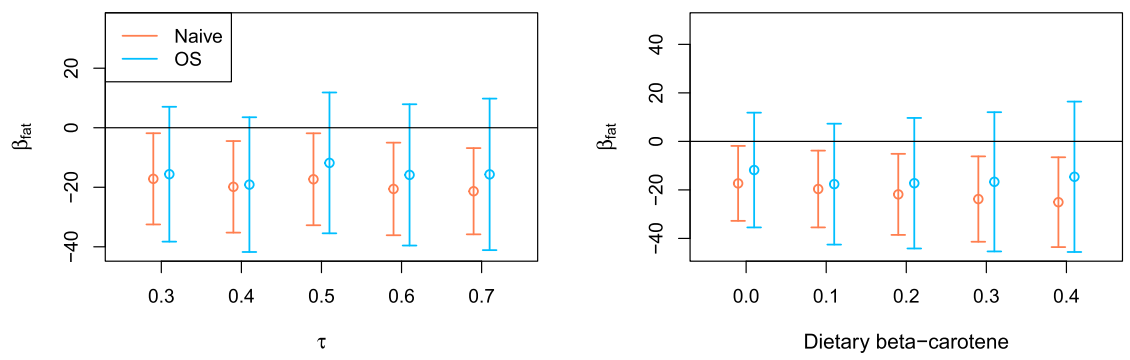

Fig 2. Inference for fat. Left: fixing scaled dietary beta-carotene level at 0, $95 \%$ confidence intervals for different $\tau$. Right: fixing $\tau=0.5,95 \%$ confidence intervals for different betacarotene levels.

of alcoholic drinks consumed per week, cholesterol consumed per day, dietary beta-carotene consumed per day and dietary retinol consumed per day.

We fit our varying coefficient model by using dietary beta-carotene consumption as the index $U$. We replace all categorical variables with dummy variables and standardize all variables. Then we include all two-way interactions in our model, so we have 116 confounding variables in total. We take the plasma betacarotene level as the outcome $Y$, the fat intake (in grams) or the fiber intake (in grams) as the treatment effect $X_{A}$ respectively, and the remaining variables as the confounding variables. We use our model to make inference on $\beta(\tau, u)$ at different beta-carotene consumption level $u$ and different quantiles $\tau$.

Our results are shown in Figures 2 and 3. The Naive method is shown in red and we compare it with the one-step correction (OS) method. From Figure 2, the result of the naive method suggests that the fat intake is significantly negatively correlated with the plasma beta-carotene level; however, the OS method suggests that this negative effect is not significant. For fiber, the Naive method underestimated the positive effect of fiber intake on the plasma beta-carotene level, whereas the OS method showed that this positive relationship is signifi- 

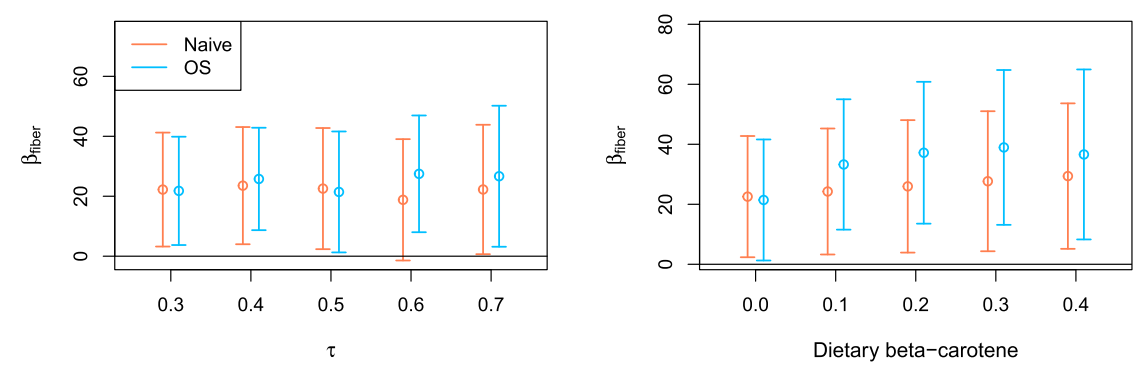

FIG 3. Inference for fiber. Left: fixing scaled dietary beta-carotene level at 0, $95 \%$ confidence intervals for different $\tau$. Right: fixing $\tau=0.5,95 \%$ confidence intervals for different betacarotene levels.

cant. Furthermore, from Figure 3 (right plot), we can see an increasing trend of the effect of fiber intake with the increasing level of dietary beta-carotene.

\section{Discussion}

We studied high-dimensional quantile regression model with varying coefficients that allows us to capture non-stationary effects of the input variables across time. Despite the importance in practical applications, no valid statistical inferential tools were previously available for this problem. We addressed this issue by developing new tools for statistical inference, allowing us to construct valid confidence bands and honest tests for nonparametric coefficient functions of time and quantile. Performing statistical inference in this regime is challenging due to the usage of model selection techniques in estimation. Our inferential results do not rely on correct model selection and are valid for a range of data generating procedures, where one cannot expect for perfect model recovery. The statistical framework allows us to construct a confidence interval at a fixed point in time and a fixed quantile based on a normal approximation, as well as a uniform confidence band for the nonparametric coefficient function based on a Gaussian process approximation. We perform numerical simulations to demonstrate the finite sample performance of our method. In addition, we also illustrate the performance of the methods through an application to a real data example.

\section{Appendix A: Technical details}

\section{A.1. Notations}

We summarize the additional notation used throughout the appendix. We let $\Psi_{\tau}(u)=\tau-\mathbb{1}\{u<0\}, \rho_{\tau}(u)=u \Psi_{\tau}(u)$, and use

$$
\begin{aligned}
W_{i}(\delta) & =\rho_{\tau}\left(y_{i}-\left(q_{i}+\delta\right)\right)-\rho_{\tau}\left(y_{i}-q_{i}\right) ; \\
W_{i}^{\#}(\delta) & =-\delta \Psi_{\tau}\left(y_{i}-q_{i}\right) ; \text { and }
\end{aligned}
$$




$$
\begin{aligned}
W_{i}^{\natural}(\delta) & =\int_{0}^{\delta}\left[\mathbb{1}\left\{y_{i} \leq q_{i}+z\right\}-\mathbb{1}\left\{y_{i} \leq q_{i}\right\}\right] d z \\
& =\left(y_{i}-\left(q_{i}+\delta\right)\right)\left[\mathbb{1}\left\{q_{i}+\delta \leq y_{i}<q_{i}\right\}-\mathbb{1}\left\{q_{i} \leq y_{i}<q_{i}+\delta\right\}\right] .
\end{aligned}
$$

We denote the sum as $\mathbb{S}_{n}[\cdot]=\sum_{i \in[n]}$. and $\mathbb{E S}_{n}[\cdot]=\mathbb{E}\left[\mathbb{S}_{n}[\cdot]\right]$. Denote the negative Hessian

$$
\begin{aligned}
H & =\mathbb{S}_{n}\left[w_{i} f_{i}\left(q_{i}\right) \cdot \Gamma_{i} \Gamma_{i}^{\top}\right] \\
H^{\star} & =\mathbb{S}_{n}\left[w_{i} f_{i}\left(\tilde{q}_{i}\right) \cdot \Gamma_{i} \Gamma_{i}^{\top}\right], \\
H(\delta) & =\mathbb{E}_{n}\left[w_{i} \hat{f}_{i}(\delta) \cdot \Gamma_{i} \Gamma_{i}^{\top}\right]=\mathbb{S}_{n}\left[w_{i} \cdot \mathbb{E}\left[\hat{f}_{i}(\delta)\right] \cdot \Gamma_{i} \Gamma_{i}^{\top}\right], \text { and } \\
\hat{H}(\delta) & =\mathbb{S}_{n}\left[w_{i} \hat{f}_{i}(\delta) \cdot \Gamma_{i} \Gamma_{i}^{\top}\right],
\end{aligned}
$$

where

$$
\hat{f}_{i}(\delta)=\frac{\mathbb{1}\left\{\left|y_{i}-\Gamma_{i}^{\top}\left(b^{\star}+\delta\right)\right| \leq h_{f}\right\}}{2 h_{f}} .
$$

Let $\Delta_{i}=\tilde{q}_{i}-q_{i}$. Recall that $V^{\star} \in \mathbb{R}^{2 k \times 2 p}$ are the rows related to $X_{A}$, $X_{A}(U-u)$ of an approximate inverse of $H^{\star}$ such that $\left\|H^{\star} V^{\star}-E_{a}\right\|_{\infty, F} \leq \lambda^{\star}$ and $\left\|V^{\star}\right\|_{F, 0} \leq s_{2}=c_{2} s$. Its estimator $\widehat{V}$ is as defined in (15). The one step correction estimator $\check{a}^{O S}=\widehat{a}-S(\widehat{a}, \widehat{c}, \widehat{V})$, where $S(a, c, V)=-\sum_{i} w_{i} V \Gamma_{i} \Psi_{\tau}\left(y_{i}-\right.$ $\left.\Gamma_{i}^{\top}\left(a^{\top}, c^{\top}\right)^{\top}\right)$.

\section{A.2. Proof of Theorem 3}

Recall the definitions of $\check{a}^{O S}$ in (17) and $S_{d}(b, V)$ in (13). We have

$$
\begin{aligned}
\check{a}^{O S}-a^{\star} & =\widehat{a}-a^{\star}-\left\{S_{d}\left(\left(\widehat{a}^{\top}, \widehat{c}^{\top}\right)^{\top}, V^{\star}\right)-S_{d}\left(\left(a^{\star \top}, c^{\star \top}\right)^{\top}, V^{\star}\right)\right\} \\
& -\left\{S_{d}\left(\left(\widehat{a}^{\top}, \widehat{c}^{\top}\right)^{\top}, \widehat{V}\right)-S_{d}\left(\left(\widehat{a}^{\top}, \widehat{c}^{\top}\right)^{\top}, V^{\star}\right)\right\}-S_{d}\left(\left(a^{\star \top}, c^{\star \top}\right)^{\top}, V^{\star}\right) .
\end{aligned}
$$

By Lemma 1 and Lemma 2 (presented later in Section A.4), we have

$$
\left\|\widehat{a}-a^{\star}-\left\{S_{d}\left(\left(\widehat{a}^{\top}, \widehat{c}^{\top}\right)^{\top}, V^{\star}\right)-S_{d}\left(\left(a^{\star \top}, c^{\star \top}\right)^{\top}, V^{\star}\right)\right\}\right\|_{2}=o_{p}\left(\sqrt{\frac{1}{n h}}\right)
$$

and

$$
\left\|S_{d}\left(\left(\widehat{a}^{\top}, \widehat{c}^{\top}\right)^{\top}, \widehat{V}\right)-S_{d}\left(\left(\widehat{a}^{\top}, \widehat{c}^{\top}\right)^{\top}, V^{\star}\right)\right\|_{2}=o_{p}\left(\sqrt{\frac{1}{n h}}\right) .
$$

Therefore,

$$
\begin{aligned}
\check{a}^{O S}-a^{\star} & =-S_{d}\left(\left(a^{\star \top}, c^{\star \top}\right), V^{\star}\right)+o_{p}\left(\sqrt{\frac{1}{n h}}\right) \\
& =\sum_{i} w_{i} V^{\star} \Gamma_{i} \Psi_{\tau}\left(y_{i}-q_{i}\right)
\end{aligned}
$$




$$
\begin{aligned}
& \quad-\sum_{i} w_{i} V^{\star} \Gamma_{i}\left\{\Psi_{\tau}\left(y_{i}-q_{i}\right)-\Psi_{\tau}\left(y_{i}-\tilde{q}_{i}\right)\right\}+o_{p}\left(\sqrt{\frac{1}{n h}}\right) \\
= & \sum_{i} w_{i} V^{\star} \Gamma_{i} \Psi_{\tau}\left(y_{i}-q_{i}\right)+o_{p}\left(\sqrt{\frac{1}{n h}}\right) .
\end{aligned}
$$

The last equality holds by Lemma 3, which we present later in Section A.4.

Because Assumptions 3 and 6 hold, by Lindeberg CLT, we have

$$
\sqrt{n h} \sum_{i} w_{i} V^{\star} \Gamma_{i} \Psi_{\tau}\left(y_{i}-q_{i}\right) \rightarrow N(0, \Sigma)
$$

and, therefore,

$$
\sqrt{n h} \Sigma^{-1 / 2}\left(\check{a}^{O S}-a^{\star}\right) \stackrel{d}{\rightarrow} N\left(0, I_{2 k}\right),
$$

where $\Sigma=\tau(1-\tau) \nu_{2} \lim _{n \rightarrow \infty} \mathbb{E}\left[\left\{V^{\star} \Gamma \Gamma^{\top} V^{\star \top}\right\} \mid U=u\right]$. By Lemma 4 (presented later in Section A.4), for both forms of $\widehat{\Sigma}_{a}$, we have

$$
\widehat{\Sigma}_{a} \stackrel{p}{\rightarrow} \Sigma
$$

Therefore by Slutsky's Theorem,

$$
\sqrt{n h} \widehat{\Sigma}_{a}^{-1 / 2}\left(\check{a}^{O S}-a^{\star}\right) \stackrel{d}{\rightarrow} N\left(0, I_{2 k}\right) .
$$

\section{A.3. Asymptotic equivalence of decorrelated score, one-step and reparameterization estimators}

Denote

$$
\begin{aligned}
& H_{a c}(f, V)=\sum_{i} w_{i} f_{i} V \Gamma_{i} \Gamma_{i}^{\top}\left[\begin{array}{ll}
0_{2(p-k) \times 2 k} & I_{2(p-k)}
\end{array}\right]^{\top}, \text { and } \\
& H_{a a}(f, V)=\sum_{i} w_{i} f_{i} V \Gamma_{i} \Gamma_{i}^{\top}\left[\begin{array}{ll}
I_{2 k} & 0_{2 k \times 2(p-k)}
\end{array}\right]^{\top} .
\end{aligned}
$$

The decorrelated score estimator $\check{a}^{D S}$ in (16) that minimizes

$$
S_{d}\left(\left(a^{\top}, \widehat{c}^{\top}\right)^{\top}, \widehat{V}\right)^{\top} \Psi^{-1} S_{d}\left(\left(a^{\top}, \widehat{c}^{\top}\right)^{\top}, \widehat{V}\right),
$$

where

$$
\Psi=\sum_{i} w_{i}^{2} \widehat{V} \Gamma_{i} \Psi_{\tau}\left(y_{i}-\Gamma_{i}^{\top} \widehat{b}\right) \Psi_{\tau}\left(y_{i}-\Gamma_{i}^{\top} \widehat{b}\right)^{\top} \Gamma_{i}^{\top} \widehat{V}^{\top},
$$

is asymptotically equivalent to the one-step estimator.

To show this, given the optimization range

$$
A_{\tau}=\left\{a:\left\|a-a^{\star}\right\|_{2}<\frac{C}{\log n}\right\},
$$


we have

$$
\left\|S_{d}\left(\left(\check{a}^{D S \top}, \widehat{c}^{\top}\right)^{\top}, \widehat{V}\right)\right\|_{2}=o_{p}\left(\sqrt{\frac{1}{n h}}\right)
$$

and

$$
\begin{aligned}
S_{d}\left(\left(\check{a}^{D S \top}, \widehat{c}^{\top}\right)^{\top}, \widehat{V}\right) & =S_{d}\left(\left(a^{\star \top}, c^{\star \top}\right)^{\top}, V^{\star}\right)+\underbrace{\left(S_{d}\left(\left(a^{\star \top}, c^{\star \top}\right)^{\top}, \widehat{V}\right)-S_{d}\left(\left(a^{\star \top}, c^{\star \top}\right)^{\top}, V^{\star}\right)\right)}_{=o_{p}\left(\sqrt{\frac{1}{n h}}\right)} \\
& +\underbrace{\left(S_{d}\left(\left(a^{\star \top}, \widehat{c}^{\top}\right)^{\top}, \widehat{V}\right)-S_{d}\left(\left(a^{\star \top}, c^{\star \top}\right)^{\top}, \widehat{V}\right)\right)}_{=o_{p}\left(\sqrt{\frac{1}{n h}}\right)} \\
& +\left(S_{d}\left(\left(\check{a}^{D S \top}, \widehat{c}^{\top}\right)^{\top}, \widehat{V}\right)-S_{d}\left(\left(a^{\star \top}, \widehat{c}^{\top}\right)^{\top}, \widehat{V}\right)\right) \\
= & S_{d}\left(\left(a^{\star \top}, c^{\star \top}\right)^{\top}, V^{\star}\right)+H_{a a}\left(f, V^{\star}\right)\left(\check{a}^{D S}-a^{\star}\right) \\
& +o\left(\left\|\check{a}^{D S}-a^{\star}\right\|_{2}\right)+o_{p}\left(\sqrt{\frac{1}{n h}}\right) .
\end{aligned}
$$

Therefore, we have

$$
\check{a}^{D S}-a^{\star}=-H_{a a}\left(f, V^{\star}\right)^{-1} S_{d}\left(\left(a^{\star \top}, c^{\star \top}\right)^{\top}, V^{\star}\right)+o_{p}\left(\sqrt{\frac{1}{n h}}\right),
$$

which is asymptotic equivalent to the one-step estimator.

For the reparameterization estimator in (18), we need to assume that $\widehat{V}$ can be decomposed as $\widehat{V}=\widehat{V}_{11}\left[\begin{array}{ll}I_{2 k} & -\widehat{v}\end{array}\right]$ where $\widehat{V}_{11}$ is invertible with high probability, and $\widehat{v}=-\widehat{V}_{11}^{-1} \widehat{V}_{12}$. Similarly we have $V^{\star}=V_{11}^{\star}\left[\begin{array}{ll}I_{2 k} & -v^{\star}\end{array}\right]$ where $v^{\star}=-\left[V_{11}^{\star}\right]^{-1} V_{12}^{\star}$. Let

$$
\begin{aligned}
& s(a, c, v)=\sum_{i} w_{i}\left[\begin{array}{ll}
I_{2 k} & -v
\end{array}\right] \Gamma_{i} \Psi_{\tau}\left(y_{i}-\Gamma_{i}^{\top}(a, c)\right), \\
& h_{a c}(f, v)=\sum_{i} w_{i} f_{i}\left[\begin{array}{ll}
I_{2 k} & -v
\end{array}\right] \Gamma_{i} \Gamma_{i}^{\top}\left[\begin{array}{ll}
0_{2(p-k) \times 2 k} & I_{2(p-k)}
\end{array}\right]^{\top}, \text { and } \\
& h_{a a}(f, v)=\sum_{i} w_{i} f_{i}\left[\begin{array}{ll}
I_{2 k} & -v
\end{array}\right] \Gamma_{i} \Gamma_{i}^{\top}\left[\begin{array}{ll}
I_{2 k} & 0_{2 k \times 2(p-k)}
\end{array}\right]^{\top} .
\end{aligned}
$$

We have $\check{a}^{R P}$ minimizing $\mathcal{L}\left(a, \widehat{c}+\widehat{v}^{\top}(\widehat{a}-a)\right)$ as defined in (8). The optimization of the low-dimension quantile regression will approximately solve the following score

$$
\begin{aligned}
s\left(a, \widehat{c}+\widehat{v}^{\top}\right. & (\widehat{a}-a), \widehat{v}) \\
& =s(a, \widehat{c}, \widehat{v})+h_{a c}(\hat{f}, \widehat{v}) \widehat{v}^{\top}(\widehat{a}-a)+o\left(\left\|\widehat{v}^{\top}(\widehat{a}-a)\right\|_{2}\right)+o_{p}\left(\sqrt{\frac{1}{n h}}\right)
\end{aligned}
$$


in the sense that

$$
s\left(\check{a}^{R P}, \widehat{c}+\widehat{v}^{\top}(\widehat{a}-\check{a}), \widehat{V}\right)=o_{p}\left(\sqrt{\frac{1}{n h}}\right) .
$$

Since

$$
h_{a c}(\hat{f}, \widehat{v}) \widehat{v}^{\top}(\widehat{a}-a)=o_{p}\left(\sqrt{\frac{1}{n h}}\right)
$$

the equivalence of $\widehat{v}$ and the lasso estimator from a regression of $\Gamma_{1: 2 k}$ on $\Gamma_{(2 k+1): 2 p}$ implies that a similar expansion as decorrelated score (13) holds. Therefore, we have

$$
\begin{aligned}
& s\left(\check{a}^{R P}, \widehat{c}, \widehat{v}\right) \\
& \quad=s\left(a^{\star}, c^{\star}, v^{\star}\right)+h_{a a}\left(f, v^{\star}\right)\left(\check{a}^{R P}-a^{\star}\right)+o\left(\left\|\check{a}^{R P}-a^{\star}\right\|_{2}\right)+o_{p}\left(\sqrt{\frac{1}{n h}}\right)
\end{aligned}
$$

and $\check{a}^{R P}-a^{\star}$ is asymptotically equivalent to $\left[h_{a a}\left(f, v^{\star}\right)\right]^{-1} s\left(a^{\star}, c^{\star}, v^{\star}\right)$, which converges to a normal distribution.

\section{A.4. Lemmas for the normality results}

Lemma 1. Suppose that Assumptions 1-6 and the conditions (22)-(26) hold. Then

$$
\left\|\widehat{a}-a^{\star}-\left\{S_{d}\left(\left(\widehat{a}^{\top}, \widehat{c}^{\top}\right)^{\top}, V^{\star}\right)-S_{d}\left(\left(a^{\star \top}, c^{\star \top}\right)^{\top}, V^{\star}\right)\right\}\right\|_{2}=o_{p}\left(\sqrt{\frac{1}{n h}}\right) .
$$

Proof. From the definition of $S_{d}(b, V)$ in (13), we can rewrite the objective as

$$
\begin{aligned}
\widehat{a} & -a^{\star}-\left\{S_{d}\left(\left(\widehat{a}^{\top}, \widehat{c}^{\top}\right)^{\top}, V^{\star}\right)-S_{d}\left(\left(a^{\star \top}, c^{\star \top}\right)^{\top}, V^{\star}\right)\right\} \\
= & \widehat{a}-a^{\star}+\sum_{i} w_{i} V^{\star} \Gamma_{i} \Psi_{\tau}\left(y_{i}-\Gamma_{i}^{\top}\left(\widehat{a}^{\top}, \widehat{c}^{\top}\right)^{\top}\right) \\
& -\sum_{i} w_{i} V^{\star} \Gamma_{i} \Psi_{\tau}\left(y_{i}-\Gamma_{i}^{\top}\left(a^{\star \top}, c^{\star \top}\right)^{\top}\right) \\
= & \widehat{a}-a^{\star}+\sum_{i} w_{i} V^{\star} \Gamma_{i}\left[\mathbb{1}\left\{y_{i} \leq \Gamma_{i} b^{\star}\right\}-\mathbb{1}\left\{y_{i} \leq \Gamma_{i} \widehat{b}\right\}\right] \\
= & \underbrace{\widehat{a}-a^{\star}+\sum_{i} w_{i} V^{\star} \Gamma_{i}\left[F_{i}\left(y_{i} \leq \Gamma_{i} b^{\star}\right)-F_{i}\left(y_{i} \leq \Gamma_{i} \widehat{b}\right)\right]}_{I} \\
+ & \underbrace{\sum_{i} w_{i} V^{\star} \Gamma_{i}\left[\left(\mathbb{1}\left\{y_{i} \leq \Gamma_{i} b^{\star}\right\}-F_{i}\left(y_{i} \leq \Gamma_{i} b^{\star}\right)\right)-\left(\mathbb{1}\left\{y_{i} \leq \Gamma_{i} \widehat{b}\right\}-F_{i}\left(y_{i} \leq \Gamma_{i} \widehat{b}\right)\right)\right]}_{I I} .
\end{aligned}
$$


For the term $I$, we use Taylor expansion and have

$$
\begin{aligned}
I & =\widehat{a}-a^{\star}+\sum_{i} w_{i} V^{\star} \Gamma_{i}\left(F_{i}\left(y_{i} \leq \Gamma_{i} b^{\star}\right)-F_{i}\left(y_{i} \leq \Gamma_{i} \widehat{b}\right)\right) \\
& =\widehat{a}-a^{\star}+\sum_{i} w_{i} V^{\star} \Gamma_{i}\left(f_{i}\left(\Gamma_{i} b^{\star}\right) \Gamma_{i}\left(b^{\star}-\widehat{b}\right)+\frac{f_{i}^{\prime}\left(\Gamma_{i} \widetilde{b}_{i}\right)}{2}\left(b^{\star}-\widehat{b}\right)^{\top} \Gamma_{i}^{\top} \Gamma_{i}\left(b^{\star}-\widehat{b}\right)\right) \\
& =\widehat{a}-a^{\star}+V^{\star} H^{\star}\left(b^{\star}-\widehat{b}\right)+R_{1} \\
& =\widehat{a}-a^{\star}+E_{a}\left(b^{\star}-\widehat{b}\right)+\left(V^{\star} H^{\star}-E_{a}\right)\left(b^{\star}-\widehat{b}\right)+R_{1} \\
& =\left(V^{\star} H^{\star}-E_{a}\right)\left(b^{\star}-\widehat{b}\right)+R_{1},
\end{aligned}
$$

where $\widetilde{b}_{i}=t_{i} b^{\star}+\left(1-t_{i}\right) \widehat{b}$ and

$$
R_{1}=\sum_{i} w_{i} V^{\star} \Gamma_{i} \frac{f_{i}^{\prime}\left(\Gamma_{i} \widetilde{b}_{i}\right)}{2}\left(b^{\star}-\widehat{b}\right)^{\top} \Gamma_{i}^{\top} \Gamma_{i}\left(b^{\star}-\widehat{b}\right) .
$$

By $(22)$

$$
\left\|R_{1}\right\|_{2} \leq 2 k B_{V} \bar{f}^{\prime} \sum_{i} w_{i}\left|\Gamma_{i}\left(b^{\star}-\widehat{b}\right)\right|^{2}=O_{p}\left(\frac{s B_{V} \log (n p)}{n h}\right) .
$$

We also have

$$
\begin{aligned}
& \left\|\left(V^{\star} H^{\star}-E_{a}\right)\left(b^{\star}-\widehat{b}\right)\right\|_{2} \\
& \quad \leq\left\|\left(V^{\star} H^{\star}-E_{a}\right)\right\|_{\infty, F}\left\|b^{\star}-\widehat{b}\right\|_{1,2}=O_{p}\left(\lambda^{\star} \cdot s \sqrt{\frac{\log (n p)}{n h}}\right),
\end{aligned}
$$

where the norm $\|\cdot\|_{\infty, F}$ is defined as

$$
\|V\|_{\infty, F}=\sup _{i \in[k], j \in[p]}\left\|V_{(i, i+k),(j, j+p)}\right\|_{F}
$$

and the second inequality is because of Assumption 5 and (22). Furthermore, since $\lambda^{\star}=O\left(B_{V} \sqrt{\frac{\log p}{n h h_{f}}}\right)$ by Assumption 5, we have

$$
\|I\|_{2}=O_{p}\left(\lambda^{\star} \cdot s \sqrt{\frac{\log (n p)}{n h}}\right)=O_{p}\left(\frac{B_{V} s \log (n p)}{n h \sqrt{h_{f}}}\right)=o_{p}\left(\sqrt{\frac{1}{n h}}\right) .
$$

For the term II, by Lemma 5 (presented later in Section A.4) we have

$$
\|I I\|_{2}=O_{p}\left(B_{K} B_{V} \sqrt{\bar{f} B_{X} \frac{m r_{b} \log p}{n h}}\right) .
$$

Plugging the rate for $r_{b}$ from condition (23), $\|I I\|_{2}=o_{p}\left(\sqrt{\frac{1}{n h}}\right)$, which completes the proof.

Lemma 2. Suppose Assumptions 1-7 and conditions (22)-(26) hold. Then

$$
\begin{aligned}
\left\|S_{d}\left(\left(\widehat{a}^{\top}, \widehat{c}^{\top}\right)^{\top}, \widehat{V}\right)-S_{d}\left(\left(\widehat{a}^{\top}, \widehat{c}^{\top}\right)^{\top}, V^{\star}\right)\right\|_{2} & \\
& =O_{p}\left(\frac{s B_{V} \log (n p)}{n h \sqrt{h_{f}}}\right)=o_{p}\left(\sqrt{\frac{1}{n h}}\right) .
\end{aligned}
$$


Proof. Using the Hölder's inequality, we have

$$
\begin{aligned}
& \left\|S_{d}\left(\left(\widehat{a}^{\top}, \widehat{c}^{\top}\right)^{\top}, \widehat{V}\right)-S_{d}\left(\left(\widehat{a}^{\top}, \widehat{c}^{\top}\right)^{\top}, V^{\star}\right)\right\|_{2} \\
& \leq\left\|\widehat{V}-V^{\star}\right\|_{1, F}\left\|\sum_{i} w_{i} \Gamma_{i} \Psi_{\tau}\left(y_{i}-\Gamma_{i}^{\top}\left(\widehat{a}^{\top}, \widehat{c}^{\top}\right)^{\top}\right)\right\|_{\infty, 2} \\
& =\left\|\widehat{V}-V^{\star}\right\|_{1, F}\left\|\sum_{i} w_{i} \Gamma_{i} \Psi_{\tau}\left(y_{i}-\Gamma_{i}^{\top} \widehat{b}\right)\right\|_{\infty, 2} \\
& \leq\left\|\widehat{V}-V^{\star}\right\|_{1, F} \cdot\left[\left\|\sum_{i} w_{i} \Gamma_{i} \Psi_{\tau}\left(y_{i}-q_{i}\right)\right\|_{\infty, 2}\right. \\
& \left.+\left\|\sum_{i} w_{i} \Gamma_{i}\left[\Psi_{\tau}\left(y_{i}-\Gamma_{i}^{\top} \widehat{b}\right)-\Psi_{\tau}\left(y_{i}-q_{i}\right)\right]\right\|_{\infty, 2}\right] .
\end{aligned}
$$

Note that

$$
\left\|\widehat{V}-V^{\star}\right\|_{1, F}=O_{p}\left(s B_{V} \sqrt{\frac{\log (n p)}{n h h_{f}}}\right)
$$

by (26) and

$$
\left\|\sum_{i} w_{i} \Gamma_{i} \Psi_{\tau}\left(y_{i}-q_{i}\right)\right\|_{\infty, 2}=O_{p}\left(\sqrt{\frac{\log (p)}{n h}}\right)
$$

by (44) and (45) in Lemma 8 (presented later in section A.5). Furthermore,

$$
\begin{aligned}
& \left\|\sum_{i} w_{i} \Gamma_{i}\left[\Psi_{\tau}\left(y_{i}-\Gamma_{i}^{\top} \widehat{b}\right)-\Psi_{\tau}\left(y_{i}-q_{i}\right)\right]\right\|_{\infty, 2} \\
& \leq\left\|\sum_{i} w_{i} \Gamma_{i}\left[\Psi_{\tau}\left(y_{i}-\Gamma_{i}^{\top} \widehat{b}\right)-\Psi_{\tau}\left(y_{i}-\Gamma_{i}^{\top} b^{\star}\right)\right]\right\|_{\infty, 2} \\
& \quad+\left\|\sum_{i} w_{i} \Gamma_{i}\left[\Psi_{\tau}\left(y_{i}-\Gamma_{i}^{\top} b^{\star}\right)-\Psi_{\tau}\left(y_{i}-q_{i}\right)\right]\right\|_{\infty, 2} .
\end{aligned}
$$

The first term in the last inequality can be bounded as

$$
\begin{aligned}
& \left\|\sum_{i} w_{i} \Gamma_{i}\left[\Psi_{\tau}\left(y_{i}-\Gamma_{i}^{\top} \widehat{b}\right)-\Psi_{\tau}\left(y_{i}-\Gamma_{i}^{\top} b^{\star}\right)\right]\right\|_{\infty, 2} \\
& =\left\|\sum_{i} w_{i} \Gamma_{i}\left(\mathbb{1}\left\{y_{i} \leq \Gamma_{i} b^{\star}\right\}-\mathbb{1}\left\{y_{i} \leq \Gamma_{i} \widehat{b}\right\}\right)\right\|_{\infty, 2} \\
& \leq\left\|\sum_{i} w_{i} \Gamma_{i}\left(F_{i}\left(y_{i} \leq \Gamma_{i} b^{\star}\right)-F_{i}\left(y_{i} \leq \Gamma_{i} \widehat{b}\right)\right)\right\|_{\infty, 2} \\
& \left.\quad+\| \sum_{i} w_{i} \Gamma_{i}\left(\mathbb{1}\left\{y_{i} \leq \Gamma_{i} b^{\star}\right\}-F_{i}\left(y_{i} \leq \Gamma_{i} b^{\star}\right)\right)-\left(\mathbb{1}\left\{y_{i} \leq \Gamma_{i} \widehat{b}\right\}-F_{i}\left(y_{i} \leq \Gamma_{i} \widehat{b}\right)\right)\right) \|_{\infty, 2} \\
& =O_{p}\left(\frac{s \log (n p)}{n h \sqrt{h_{f}}}\right)+O_{p}\left(B_{K} B_{V} \sqrt{\bar{f} B_{X} \frac{m r_{b} \log (n p)}{n h}}\right)
\end{aligned}
$$

where the first part of the last equation is the same as the proof in Lemma 1 and the second part comes from Lemma 5 (presented later in Section A.4), where $r_{b} \leq$ $O_{p}\left(s \sqrt{\frac{\log (n p)}{n h}}\right)$ because of (23) and $B_{V} \asymp O(\log p)$. 
For the second term, applying Lemma 3 (presented next) with a union bound, we have

$$
\left\|\sum_{i} w_{i} \Gamma_{i}\left[\Psi_{\tau}\left(y_{i}-\Gamma_{i}^{\top} b^{\star}\right)-\Psi_{\tau}\left(y_{i}-q_{i}\right)\right]\right\|_{\infty, 2}=o_{p}\left(\sqrt{\frac{\log (n p)}{n h}}\right) .
$$

Combining the two bounds, we have

$$
\begin{aligned}
\left\|S_{d}\left(\left(\widehat{a}^{\top}, \widehat{c}^{\top}\right)^{\top}, \widehat{V}\right)-S_{d}\left(\left(\widehat{a}^{\top}, \widehat{c}^{\top}\right)^{\top}, V^{\star}\right)\right\|_{F} \\
=O_{p}\left(s B_{V} \sqrt{\frac{\log (n p)}{n h h_{f}}}\right) . \\
\quad\left\{O_{p}\left(\frac{s \log (n p)}{n h \sqrt{h_{f}}}\right)+O_{p}\left(\sqrt{\frac{s(\log (n p))^{7 / 2}}{(n h)^{3 / 2}}}\right)+o_{p}\left(\sqrt{\frac{\log (n p)}{n h}}\right)\right\} \\
=o_{p}\left(\sqrt{\frac{1}{n h}}\right) .
\end{aligned}
$$

The last equality is because of Assumption 7. This completes the proof.

Lemma 3. Under Assumptions 1, 2, 4, and 6, for any $V \in \mathbb{C}\left(S_{2}\right) \subset \mathbb{R}^{2 k \times 2 p}$ such that $\max _{i \in[n]}\left\|V \Gamma_{i}\right\|_{2}=O(\log p) \leq B_{V}$ and $h \leq O\left(n^{-1 / 3}\right)$ as assumed in Assumption \%, we have

$$
\begin{aligned}
\left\|\sum_{i} w_{i} V \Gamma_{i}\left[\Psi_{\tau}\left(y_{i}-q_{i}\right)-\Psi_{\tau}\left(y_{i}-\tilde{q}_{i}\right)\right]\right\|_{2} \\
=O_{p}\left(\sqrt{B_{V} B_{K} \frac{\bar{f}}{n h} \cdot\left(h^{2}+\epsilon_{R}\right)}+\frac{\bar{f} \kappa_{+}\|V\|_{F}}{n h} \cdot\left(h^{2}+\epsilon_{R}\right)\right) .
\end{aligned}
$$

Proof. We have

$$
\begin{gathered}
\left\|\mathbb{S}_{n}\left[w_{i} V \Gamma_{i}\left[\Psi_{\tau}\left(y_{i}-q_{i}\right)-\Psi_{\tau}\left(y_{i}-\tilde{q}_{i}\right)\right]\right]\right\|_{2} \\
=\left\|\mathbb{S}_{n}\left[w_{i} V \Gamma_{i}\left[\mathbb{1}\left\{y_{i} \leq \tilde{q}_{i}\right\}-\mathbb{1}\left\{y_{i} \leq q_{i}\right\}\right]\right]\right\|_{2} \\
\leq \underbrace{\left\|\left(\mathbb{S}_{n}-\mathbb{E}_{n}\right)\left[w_{i} V \Gamma_{i}\left(\mathbb{1}\left\{y_{i} \leq \tilde{q}_{i}\right\}-\mathbb{1}\left\{y_{i} \leq q_{i}\right\}\right)\right]\right\|_{2}}_{I} \\
+\underbrace{\left\|\mathbb{S}_{n}\left[w_{i} V \Gamma_{i}\left[F_{i}\left(\tilde{q}_{i}\right)-F_{i}\left(q_{i}\right)\right]\right]\right\|_{2}}_{I I} .
\end{gathered}
$$

By Lemma 6 (presented later in this section),

$$
I=O_{p}\left(B_{V} B_{K} \sqrt{\frac{\bar{f}}{n h} \cdot\left(h^{2}+\epsilon_{R}\right)}\right) .
$$

For the term II, using the mean value theorem and the Cauchy-Schwarz inequality, we have 


$$
\begin{array}{r}
I I \leq \bar{f} \sqrt{\sum_{i} w_{i} \operatorname{trace}\left(V \Gamma_{i} \Gamma_{i}^{\top} V^{\top}\right)} \cdot \sqrt{\sum_{i} w_{i}\left(\tilde{q}_{i}-q_{i}\right)^{2}} \\
=O_{p}\left(\bar{f} \kappa_{+}\|V\|_{F} \cdot\left(h^{2}+\epsilon_{R}\right)\right),
\end{array}
$$

where the last equality is because of Lemma 12 and Assumption 6. The proof follows from the rate of $h$ given in Assumption 7.

Lemma 4. Let

$$
\begin{aligned}
& \widehat{\Sigma}_{a 1}:=n h \sum_{i} w_{i}^{2} \widehat{V} \Gamma_{i} \Psi_{\tau}\left(y_{i}-\Gamma_{i}^{\top} \widehat{b}\right) \Psi_{\tau}\left(y_{i}-\Gamma_{i}^{\top} \widehat{b}\right)^{\top} \Gamma_{i}^{\top} \widehat{V}^{\top}, \\
& \widehat{\Sigma}_{a 2}:=\tau(1-\tau) \nu_{2} \widehat{V}\left\{\sum_{j} w_{j} \Gamma_{j} \Gamma_{j}^{\top}\right\} \widehat{V}^{\top},
\end{aligned}
$$

and

$$
\Sigma=\tau(1-\tau) \nu_{2} \lim _{n \rightarrow \infty} \mathbb{E}\left[V^{\star} \Gamma \Gamma^{\top} V^{\star \top} \mid U=u\right] .
$$

Then $\widehat{\Sigma}_{a i} \stackrel{p}{\rightarrow} \Sigma$ for $i=1,2$.

Proof. From the consistency of $\widehat{V}$ and $\widehat{b}$, we have $\left\|\widehat{V}-V^{\star}\right\|_{F}=o_{p}(1)$ and

$$
\max _{i}\left|\Psi_{\tau}\left(y_{i}-\Gamma_{i}^{\top} \widehat{b}\right)-\Psi_{\tau}\left(y_{i}-q_{i}\right)\right|=o_{p}(1) .
$$

Therefore,

$$
\begin{aligned}
\widehat{\Sigma}_{a 1} & =n h \sum_{i} w_{i}^{2} \widehat{V} \Gamma_{i} \Psi_{\tau}\left(y_{i}-\Gamma_{i}^{\top} \widehat{b}\right) \Psi_{\tau}\left(y_{i}-\Gamma_{i}^{\top} \widehat{b}\right)^{\top} \Gamma_{i}^{\top} \widehat{V}^{\top} \\
& =n h \sum_{i} w_{i}^{2} V^{\star} \Gamma_{i} \Psi_{\tau}\left(y_{i}-\Gamma_{i}^{\top} \widehat{b}\right) \Psi_{\tau}\left(y_{i}-\Gamma_{i}^{\top} \widehat{b}\right)^{\top} \Gamma_{i}^{\top} V^{\star \top}+o_{p}(1) \\
& =(n h)^{-1} \sum_{i} K^{2}\left(\frac{U_{i}-u}{h}\right) V^{\star} \Gamma_{i} \Psi_{\tau}\left(y_{i}-\Gamma_{i}^{\top} \widehat{b}\right) \Psi_{\tau}\left(y_{i}-\Gamma_{i}^{\top} \widehat{b}\right)^{\top} \Gamma_{i}^{\top} V^{\star \top}+o_{p}(1) \\
& =\mathbb{E}\left[(n h)^{-1} \sum_{i} K^{2}\left(\frac{U_{i}-u}{h}\right) V^{\star} \Gamma_{i} \Psi_{\tau}\left(y_{i}-q_{i}\right) \Psi_{\tau}\left(y_{i}-q_{i}\right)^{\top} \Gamma_{i}^{\top} V^{\star \top}\right]+o_{p}(1) \\
& =\Sigma+o_{p}(1),
\end{aligned}
$$

which shows (30),

From the condition (55), we have $\left\|\widehat{V}-V^{\star}\right\|_{F}=o_{p}(1)$. By the strong law of large numbers,

$$
\left\|\sum_{j} w_{j} V^{\star} \Gamma_{j} \Gamma_{j}^{\top} V^{\star \top}-\mathbb{E}\left[V^{\star} \Gamma \Gamma^{\top} V^{\star \top} \mid U=u\right]\right\|_{F}=o_{p}(1) .
$$

Then by the continuous mapping theorem, we have $\left\|\widehat{\Sigma}_{a 2}-\Sigma\right\|_{F}=o_{p}(1)$. This shows (31). The proof is complete now. 
Lemma 5. Suppose Assumptions 1, 2, 3, and 6 hold. For any $V \in \mathbb{C}\left(S_{2}\right) \subset$ $\mathbb{R}^{2 k \times 2 p}$ that satisfies $\max _{i \in[n]}\left\|V \Gamma_{i}\right\|_{2}=B_{V}=O(\log p)$ and $r_{b} \asymp s \sqrt{\frac{\log (n p)}{n h}}$, we have

$$
\begin{aligned}
& \sup _{\substack{\|\delta\|_{0,2} \leq m \\
\|\delta\|_{1,2} \leq r_{b}}}\left\|\left(\mathbb{S}_{n}-\mathbb{E} \mathbb{S}_{n}\right)\left[w_{i} V \Gamma_{i}\left(\mathbb{1}\left\{y_{i} \leq \Gamma_{i}^{\top} b^{\star}\right\}-\mathbb{1}\left\{y_{i} \leq \Gamma_{i}^{\top}\left(b^{\star}+\delta\right)\right\}\right)\right]\right\|_{2} \\
&=O_{p}\left(B_{K} B_{V} \sqrt{\bar{f} B_{X} \frac{m r_{b} \log (n p)}{n h}}\right) .
\end{aligned}
$$

Proof. Let $\mathcal{W}=\left\{\tilde{W}_{1}, \ldots, \tilde{W}_{K}\right\}$ be the $\frac{1}{2}$-net for $\left\{W \in \mathbb{R}^{2 k} \mid\|W\|_{2} \leq 1\right\}$. That is, for all $W \in \mathbb{R}^{2 k}$ with $\|W\|_{2} \leq 1$, there exists

$$
\tilde{W} \in \mathcal{W} \subseteq\left\{W \in \mathbb{R}^{2 k} \mid\|W\|_{2} \leq 1\right\}
$$

such that $\|\tilde{W}-W\|_{2} \leq \frac{1}{2}$. We have that $K \leq 5^{2 k}$. Then

$$
\begin{aligned}
& \sup _{\substack{\|\delta\|_{0,2} \leq m \\
\|\delta\|_{1,2} \leq r_{b}}}\left\|\left(\mathbb{S}_{n}-\mathbb{E S}_{n}\right)\left[w_{i} \cdot V \Gamma_{i}\left(\mathbb{1}\left\{y_{i} \leq \Gamma_{i}^{\top} b^{\star}\right\}-\mathbb{1}\left\{y_{i} \leq \Gamma_{i}^{\top}\left(b^{\star}+\delta\right)\right\}\right)\right]\right\|_{2} \\
& \leq 2 \cdot \max _{\tilde{W} \in \mathcal{W}} \sup _{\|\delta\|_{0,2} \leq m} \\
& \qquad \delta \|_{1,2} \leq r_{b} \\
& \left(\mathbb{S}_{n}-\mathbb{E S}_{n}\right)\left[w_{i} \cdot\left(\left|\mathbb{1}\left\{y_{i} \leq \Gamma_{i}^{\top} b^{\star}\right\}-\mathbb{1}\left\{y_{i} \leq \Gamma_{i}^{\top}\left(b^{\star}+\delta\right)\right\}\right|\right) \cdot\left|\tilde{W}^{\top} V \Gamma_{i}\right|\right] .
\end{aligned}
$$

For the expectation, we have

$$
\begin{aligned}
\mathbb{E}\left[\mid \mathbb{1}\left\{y_{i} \leq \Gamma_{i}^{\top}\right.\right. & \left.\left.b^{\star}\right\}-\mathbb{1}\left\{y_{i} \leq \Gamma_{i}^{\top}\left(b^{\star}+\delta\right)\right\} \mid\right] \\
& \leq \mathbb{E}\left[-\left|\Gamma_{i}^{\top} \delta\right| \leq y_{i}-\Gamma_{i}^{\top} b^{\star} \leq\left|\Gamma_{i}^{\top} \delta\right|\right] \\
& =F_{i}\left(\Gamma_{i}^{\top} b^{\star}+\left|\Gamma_{i}^{\top} \delta\right|\right)-F_{i}\left(\Gamma_{i}^{\top} b^{\star}-\left|\Gamma_{i}^{\top} \delta\right|\right) \\
& \leq 2 \bar{f} \cdot\left|\Gamma_{i}^{\top} \delta\right| \\
& \leq 2 \bar{f} B_{X} r_{b} .
\end{aligned}
$$

For a fixed $\tilde{W} \in \mathcal{W}$ and $|S| \leq m$, define

$$
\begin{aligned}
a_{i} & =w_{i} \cdot\left|\tilde{W}^{\top} V \Gamma_{i}\right|, \text { and } \\
\mathcal{G}_{S}=\left\{\left(y_{i}, x_{i}, u_{i}\right) \mapsto a_{i} \cdot \mathbb{1}\left\{-\left|\Gamma_{i}^{\top} \delta\right| \leq y_{i}-\Gamma_{i}^{\top} b^{\star} \leq\left|\Gamma_{i}^{\top} \delta\right|\right\}:\right. & \left.\quad \operatorname{support}(\delta)=S,\|\delta\|_{2} \leq r_{b}\right\} . \\
\mathcal{G}=\cup_{S:|S| \leq m} \mathcal{G}_{S} &
\end{aligned}
$$

Let $G(\cdot)=(n h)^{-1} B_{K}\left(\tilde{W}^{\top} V \Gamma_{i}\right)$ be an envelope of $\mathcal{G}$. Then $\|G\|_{\infty} \leq \frac{B_{K} B_{V}}{n h}$. For a fixed $g \in \mathcal{G}$, let

$$
g_{i}=g\left(y_{i}, x_{i}, u_{i}\right)=a_{i} \cdot \mathbb{1}\left\{-\left|\Gamma_{i}^{\top} \delta\right| \leq y_{i}-\Gamma_{i}^{\top} b^{\star} \leq\left|\Gamma_{i}^{\top} \delta\right|\right\} .
$$


Therefore, the variance is bounded as

$$
\sigma_{\mathcal{G}}^{2} \leq \sup _{g \in \mathcal{G}} \sum_{i \in[n]} \mathbb{E}\left[g_{i}^{2}\right] \lesssim \bar{f} B_{X} r_{b} \sum_{i \in[n]} a_{i}^{2} \lesssim \frac{\bar{f} B_{X} B_{K}^{2} B_{V}^{2}}{n h} \cdot r_{b},
$$

since

$$
\sum_{i \in[n]} a_{i}^{2} \leq B_{V}^{2} \sum_{i \in[n]} w_{i}^{2} \leq \frac{B_{K}^{2} B_{V}^{2}}{n h}
$$

The VC dimension for the space

$$
\mathcal{F}_{S}=\left\{\left(y_{i}, x_{i}, u_{i}\right) \mapsto \mathbb{1}\left\{-\left|\Gamma_{i}^{\top} \delta\right| \leq y_{i}-\Gamma_{i}^{\top} b^{\star} \leq\left|\Gamma_{i}^{\top} \delta\right|\right\}: \operatorname{support}(\delta)=S,\|\delta\|_{2} \leq r_{b}\right\}
$$

is $O(m)$. Therefore, applying Lemma 22 (presented later in Section A.8),

$$
\sup _{Q} N\left(\epsilon \cdot \frac{B_{K} B_{V}}{n h}, \mathcal{G}_{S},\|\cdot\|_{L_{2}(Q)}\right) \leq\left(\frac{C}{\epsilon}\right)^{c m},
$$

and

$$
\sup _{Q} N\left(\epsilon \cdot \frac{B_{K} B_{V}}{n h}, \mathcal{G},\|\cdot\|_{L_{2}(Q)}\right) \leq\left(\frac{C}{\epsilon}\right)^{c m} \cdot p^{m} .
$$

Applying Lemma 21 (presented later in Section A.7) with $\sigma_{\mathcal{G}}=B_{K} B_{V} \sqrt{\frac{\bar{f} B_{X} r_{b}}{n h}}$, $\|G\|_{\infty} \leq \frac{B_{K} B_{V}}{n h}, V=c m, U=\frac{B_{K} B_{V}}{n h}$, and $A=C m^{1 / c m} p^{1 / c}$ then gives us

$$
\begin{aligned}
& \mathbb{E}\left[\sup _{g \in \mathcal{G}} \sum_{i \in[n]} g_{i}-\mathbb{E}\left[g_{i}\right]\right] \\
& \leq\left(c m \frac{B_{K} B_{V}}{n h} \log \left(\frac{C p^{1 / c}}{\sqrt{\bar{f} B_{X} r_{b} h}}\right)+B_{K} B_{V} \sqrt{\frac{\bar{f} B_{X} r_{b}}{n h}} \sqrt{c m \log \left(\frac{C p^{1 / c}}{\sqrt{\bar{f} B_{X} r_{b} h}}\right)}\right) \\
& =O\left(B_{K} B_{V} \sqrt{\bar{f} B_{X} \frac{m r_{b} \log (p \vee n)}{n h}}\right),
\end{aligned}
$$

under the conditions of the lemma and the growth condition in Assumption 7. Lemma 25 then gives us

$$
\sup _{g \in \mathcal{G}} \sum_{i \in[n]} g_{i}-\mathbb{E}\left[g_{i}\right]=O_{p}\left(B_{K} B_{V} \sqrt{\bar{f} B_{X} \frac{m r_{b} \log (n p)}{n h}}\right) .
$$

A union bound over $\tilde{W} \in \mathcal{W}$ concludes the proof.

Lemma 6. Suppose Assumptions 1, 2, 3, 4, 6 and 7 hold. For all $V \in \mathbb{C}\left(S_{2}\right) \subset$ $\mathbb{R}^{2 k \times 2 p}$ with $B_{V}=\max _{i \in[n]}\left\|V \Gamma_{i}\right\|_{2}$, we have

$$
\begin{aligned}
\|\left(\mathbb{S}_{n}-\mathbb{E S}_{n}\right)\left[w _ { i } V \Gamma _ { i } \left(\mathbb{1}\left\{y_{i} \leq \tilde{q}_{i}\right\}-\mathbb{1}\left\{y_{i}\right.\right.\right. & \left.\left.\left.\leq q_{i}\right\}\right)\right] \|_{2} \\
& =O_{P}\left(B_{K} B_{V} \sqrt{\frac{\bar{f}}{n h} \cdot\left(h^{2}+\epsilon_{R}\right)}\right)
\end{aligned}
$$


Proof. Let $\mathcal{W}=\left\{\tilde{W}_{1}, \ldots, \tilde{W}_{K}\right\}$ be the $\frac{1}{2}$-net for $\left\{W \in \mathbb{R}^{2 k} \mid\|W\|_{2} \leq 1\right\}$. We have that $K \leq 5^{2 k}$ and

$$
\begin{aligned}
& \left\|\left(\mathbb{S}_{n}-\mathbb{E S}_{n}\right)\left[w_{i} \cdot V \Gamma_{i}\left(\mathbb{1}\left\{y_{i} \leq \tilde{q}_{i}\right\}-\mathbb{1}\left\{y_{i} \leq q_{i}\right\}\right)\right]\right\|_{2} \\
& \quad \leq 2 \cdot \max _{\tilde{W} \in \mathcal{W}}\left(\mathbb{S}_{n}-\mathbb{E} \mathbb{S}_{n}\right)\left[w_{i} \cdot\left(\left|\mathbb{1}\left\{y_{i} \leq \tilde{q}_{i}\right\}-\mathbb{1}\left\{y_{i} \leq q_{i} \mid\right\} \cdot\right| \tilde{W}^{\top} V \Gamma_{i} \mid\right)\right] .
\end{aligned}
$$

Let

$$
\begin{aligned}
& a_{i}=w_{i} \cdot\left|\tilde{W}^{\top} V \Gamma_{i}\right|, \text { and } \\
& g_{i}=g\left(y_{i}, x_{i}, u_{i}\right)=a_{i} \cdot\left(\mathbb{1}\left\{y_{i} \leq \tilde{q}_{i}\right\}-\mathbb{1}\left\{y_{i} \leq q_{i}\right\}\right) .
\end{aligned}
$$

Since

$$
\mathbb{E}\left[\left|\mathbb{1}\left\{y_{i} \leq \tilde{q}_{i}\right\}-\mathbb{1}\left\{y_{i} \leq q_{i}\right\}\right|\right] \leq 2 \bar{f} \cdot\left|q_{i}-\tilde{q}_{i}\right|,
$$

we have

$$
\begin{aligned}
& \sum_{i \in[n]} \mathbb{E}\left[g_{i}^{2}\right] \lesssim \sum_{i} 2 \bar{f} \cdot\left|q_{i}-\tilde{q}_{i}\right| a_{i}^{2}=\sum_{i} 2 \bar{f} \cdot \mid\left|q_{i}-\tilde{q}_{i}\right| w_{i}^{2}\left(\tilde{W}^{\top} V \Gamma_{i}\right)^{2} \\
& \leq \frac{\bar{f} B_{K}^{2} B_{V}^{2}}{n h} \sqrt{\mathbb{S}_{n}\left[w_{i}\left(q_{i}-\tilde{q}_{i}\right)^{2}\right]} .
\end{aligned}
$$

Then by Lemma 12 and Assumption 6, we have

$$
\sum_{i \in[n]} \mathbb{E}\left[g_{i}^{2}\right]=O_{p}\left(\frac{\bar{f} B_{K}^{2} B_{V}^{2}}{n h} \cdot\left(h^{2}+\epsilon_{R}\right)\right) .
$$

The result follows from the Bernstein's inequality and the union bound over $\mathcal{W}$.

\section{A.5. Consistency of the initial estimator $\widehat{b}^{\text {ini }}$}

We show the convergence guarantee of the initial estimator $\widehat{b}^{\text {ini }}$ defined in Section 3 Step 1 . Notice that in the following two sections, we slightly abuse the notation by denoting $\widehat{b}^{\text {ini }}$ from Section 3 as $\widehat{b}$, and $\widehat{b}$ from Section 3 is defined as $\widehat{b}^{\lambda}$, since it is obtained by thresholding at the level $\lambda$.

Let

$$
W_{i}(\delta)=\rho_{\tau}\left(y_{i}-\left(q_{i}+\delta\right)\right)-\rho_{\tau}\left(y_{i}-q_{i}\right),
$$

which can be decomposed as

$$
\begin{aligned}
W_{i}(\delta) & =-\delta \Psi_{\tau}\left(y_{i}-q_{i}\right)+\int_{0}^{\delta}\left[\mathbb{1}\left\{y_{i} \leq q_{i}+z\right\}-\mathbb{1}\left\{y_{i} \leq q_{i}\right\}\right] d z \\
& =: W_{i}^{\#}(\delta)+W_{i}^{\natural}(\delta),
\end{aligned}
$$


using the Knight's identity. Note that we can also write

$$
W_{i}^{\natural}(\delta)=\left(y_{i}-\left(q_{i}+\delta\right)\right)\left[\mathbb{1}\left\{q_{i}+\delta \leq y_{i}<q_{i}\right\}-\mathbb{1}\left\{q_{i} \leq y_{i}<q_{i}+\delta\right\}\right] .
$$

With this notation, we study properties of the following penalized quantile regression estimator

$$
\widehat{b}=\arg \min _{b} \sum_{i \in[n]} w_{i} \cdot \rho_{\tau}\left(y_{i}-\Gamma_{i}^{\top} b\right)+\lambda\|b\|_{1,2},
$$

where the groups are formed by pairs $\left(b_{0 j}, b_{1 j}\right)$ for $j \in[p]$ and

$$
w_{i}=(n h)^{-1} K\left(\frac{U_{i}-u}{h}\right) .
$$

The estimated quantile function is denoted as $\hat{q}_{i}=\Gamma_{i}^{\top} \widehat{b}$.

Theorem 4. Under Assumptions 1, 3, 4 and 6, we have

$$
\mathbb{S}_{n}\left[w_{i} \cdot\left(\Gamma_{i}^{\top}\left(\widehat{b}-b^{\star}\right)\right)^{2}\right]=O_{p}\left(\frac{s \log (n p)}{n h}\right)
$$

and

$$
\left\|\widehat{b}-b^{\star}\right\|_{1,2}=O_{p}\left(s \sqrt{\frac{\log (n p)}{n h}}\right) .
$$

Proof. Denote $S^{\prime}=\operatorname{support}\left\{b^{\star}\right\}$.

Let $r_{b}$ be a rate satisfying $r_{b}=O_{p}\left(\sqrt{\frac{s \log (n p)}{n h}}\right)$. Recall that

$$
\kappa_{q}=\inf _{\substack{\|\delta\|_{1,2} \leq \frac{7\left|S^{\prime}\right| \cdot \sqrt{\log p}}{\kappa \cdot \sqrt{n h}} \\ \mathbb{S}_{n}\left[w_{i}\left(\Gamma_{i}^{\top} \delta\right)^{2}\right]=\frac{\left|S^{\prime}\right| \log p}{n h}}} \frac{\left(\underline{f} \cdot \mathbb{S}_{n}\left[w_{i} \cdot\left(\Gamma_{i}^{\top} \delta\right)^{2}\right]\right)^{3 / 2}}{\bar{f}^{\prime} \cdot \mathbb{S}_{n}\left[w_{i} \cdot\left(\Gamma_{i}^{\top} \delta\right)^{3}\right]} .
$$

As $n$ grows, we have

$$
\kappa_{q} \geq r_{b} \sqrt{\underline{f}}
$$

In order to establish that $\mathbb{S}_{n}\left[w_{i} \cdot\left(\Gamma_{i}^{\top}\left(\widehat{b}-b^{\star}\right)\right)^{2}\right] \leq r_{b}^{2}$, we use the proof by contradiction.

Suppose that $\mathbb{S}_{n}\left[w_{i} \cdot\left(\Gamma_{i}^{\top}\left(\widehat{b}-b^{\star}\right)\right)^{2}\right]>r_{b}^{2}$. Since the objective function is convex, there exists a vector

$$
\check{b}=b^{\star}+\left(\widehat{b}-b^{\star}\right) \frac{r_{b}}{\sqrt{\mathbb{S}_{n}\left[w_{i} \cdot\left(\Gamma_{i}^{\top}\left(\widehat{b}-b^{\star}\right)\right)^{2}\right]}}
$$


such that $\mathbb{S}_{n}\left[w_{i} \cdot\left(\Gamma_{i}^{\top}\left(\check{b}-b^{\star}\right)\right)^{2}\right]=r_{b}^{2}$ and

$$
\mathbb{S}_{n}\left[w_{i} \cdot\left(\rho_{\tau}\left(y_{i}-\check{q}_{i}\right)-\rho_{\tau}\left(y_{i}-\tilde{q}_{i}\right)\right)\right] \leq \lambda\left(\|\check{b}\|_{1,2}-\left\|b^{\star}\right\|_{1,2}\right),
$$

is satisfied. We separate our analysis into two parts, according to whether $3\left\|\left(\check{b}-b^{\star}\right)_{S^{\prime}}\right\|_{1,2} \geq \frac{2}{\lambda} \mathbb{S}_{n}\left[w_{i} \cdot W_{i}^{\natural}\left(\tilde{q}_{i}-q_{i}\right)\right]$ in (39) or not.

First, suppose that $3\left\|\left(\check{b}-b^{\star}\right)_{S^{\prime}}\right\|_{1,2} \geq \frac{2}{\lambda} \mathbb{S}_{n}\left[w_{i} \cdot W_{i}^{\natural}\left(\tilde{q}_{i}-q_{i}\right)\right]$. By Lemma 7 (presented next), $\left\|\left(\check{b}-b^{\star}\right)_{N^{\prime}}\right\|_{1,2} \leq 6\left\|\left(\check{b}-b^{\star}\right)_{S^{\prime}}\right\|_{1,2}$ and

$$
\begin{aligned}
\left\|\check{b}-b^{\star}\right\|_{1,2} \leq 7\left\|\left(\check{b}-b^{\star}\right)_{S^{\prime}}\right\|_{1,2} \leq 7 & \sqrt{\left|S^{\prime}\right|}\left\|\left(\check{b}-b^{\star}\right)_{S^{\prime}}\right\|_{2} \\
& \leq \frac{7 \sqrt{\left|S^{\prime}\right|} \cdot r_{b}}{\kappa_{-}}=O_{p}\left(s \sqrt{\frac{\log (n p)}{n h}}\right) .
\end{aligned}
$$

Starting from (40), we have that

$$
\begin{aligned}
\lambda\left\|\breve{b}-b^{\star}\right\|_{1,2} \geq \mathbb{E S}_{n}[ & \left.w_{i} \cdot\left(W_{i}\left(\Gamma_{i}^{\top} \check{b}-q_{i}\right)-W_{i}\left(\Gamma_{i}^{\top} b^{\star}-q_{i}\right)\right)\right] \\
& +\left(\mathbb{S}_{n}-\mathbb{E S}_{n}\right)\left[w_{i} \cdot\left(W_{i}\left(\Gamma_{i}^{\top} \check{b}-q_{i}\right)-W_{i}\left(\Gamma_{i}^{\top} b^{\star}-q_{i}\right)\right)\right] .
\end{aligned}
$$

Lemma 10 (presented later in this section) gives us

$$
\mathbb{E S}_{n}\left[w_{i} \cdot\left(W_{i}\left(\Gamma_{i}^{\top} \check{b}-q_{i}\right)-W_{i}\left(\Gamma_{i}^{\top} b^{\star}-q_{i}\right)\right)\right] \geq \frac{f r_{b}^{2} \wedge \kappa_{q} \sqrt{\underline{f}} r_{b}}{3} \geq \frac{f r_{b}^{2}}{3},
$$

where the second inequality follows under (36). On the event $\mathcal{E}_{\mathrm{QR}}(\lambda)$, we have

$$
\left(\mathbb{S}_{n}-\mathbb{E S}_{n}\right)\left[w_{i} \cdot\left(W_{i}^{\#}\left(\Gamma_{i}^{\top} \check{b}-q_{i}\right)-W_{i}^{\#}\left(\Gamma_{i}^{\top} b^{\star}-q_{i}\right)\right)\right] \geq-\frac{\lambda}{2}\left\|\check{b}-b^{\star}\right\|_{1,2} .
$$

Lemma 9 (presented later in this section) gives us

$$
\sup _{\substack{\|\delta\|_{1,2} \leq \frac{7 \sqrt{\left|S^{\top}\right| \cdot r_{b}}}{\kappa} \\ \mathbb{S}_{n}\left[w_{i}\left(\Gamma_{i}^{\top} \delta\right)^{2}\right]=r_{b}^{2}}}\left|\left(\mathbb{S}_{n}-\mathbb{E S}_{n}\right)\left[w_{i} \cdot\left(W_{i}^{\natural}\left(\Gamma_{i}^{\top} \check{b}-q_{i}\right)-W_{i}^{\natural}\left(\Gamma_{i}^{\top} b^{\star}-q_{i}\right)\right)\right]\right|=o_{p}\left(r_{b}\right) .
$$

Putting everything together, we obtain that

$$
0 \geq \frac{f r_{b}}{3}-\frac{7 \lambda \sqrt{\left|S^{\prime}\right|}}{2 \kappa_{-}}-o_{p}\left(r_{b}\right)>0
$$

which is a contradiction.

The second part of the upper bound is established in the case when

$$
3\left\|\left(\breve{b}-b^{\star}\right)_{S^{\prime}}\right\|_{1,2}<(2 / \lambda) \mathbb{S}_{n}\left[w_{i} \cdot W_{i}^{\natural}\left(\tilde{q}_{i}-q_{i}\right)\right] .
$$

Then we have that

$$
\left\|\left(\check{b}-b^{\star}\right)_{N^{\prime}}\right\|_{1,2} \leq \frac{4}{\lambda} \mathbb{S}_{n}\left[w_{i} \cdot W_{i}^{\natural}\left(\tilde{q}_{i}-q_{i}\right)\right]
$$


and

$$
\left\|\check{b}-b^{\star}\right\|_{1,2} \leq \frac{6}{\lambda} \mathbb{S}_{n}\left[w_{i} \cdot W_{i}^{\natural}\left(\tilde{q}_{i}-q_{i}\right)\right]=O_{p}\left(s \sqrt{\frac{\log (n p)}{n h}}\right),
$$

where the last equation is because of Lemmas 8 and 11. The same argument as above gives us a contradiction, which completes the proof.

Lemma 7. On the event

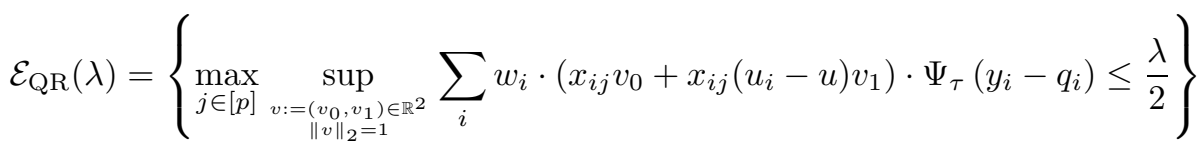

we have

$$
\left\|\left(\widehat{b}-b^{\star}\right)_{N^{\prime}}\right\|_{1,2} \leq 3\left\|\left(\widehat{b}-b^{\star}\right)_{S^{\prime}}\right\|_{1,2}+\frac{2}{\lambda} \mathbb{S}_{n}\left[w_{i} \cdot W_{i}^{\natural}\left(\tilde{q}_{i}-q_{i}\right)\right],
$$

where $N^{\prime}=S^{\prime c}$.

Proof. Our starting point is the observation that

$$
\mathbb{S}_{n}\left[w_{i} \cdot\left(\rho_{\tau}\left(y_{i}-\hat{q}_{i}\right)-\rho_{\tau}\left(y_{i}-\tilde{q}_{i}\right)\right)\right] \leq \lambda\left(\left\|b^{\star}\right\|_{1,2}-\|\widehat{b}\|_{1,2}\right),
$$

since $\widehat{b}$ minimizes $(35)$. Due to convexity of $\rho_{\tau}(\cdot)$, we have

$$
\mathbb{S}_{n}\left[w_{i} \cdot\left(\rho_{\tau}\left(y_{i}-\hat{q}_{i}\right)-\rho_{\tau}\left(y_{i}-q_{i}\right)\right)\right] \geq \mathbb{S}_{n}\left[w_{i} \cdot\left(q_{i}-\hat{q}_{i}\right) \cdot \Psi_{\tau}\left(y_{i}-q_{i}\right)\right] .
$$

Using (33), we have

$$
\begin{aligned}
\mathbb{S}_{n}\left[w _ { i } \cdot \left(\rho _ { \tau } \left(y_{i}\right.\right.\right. & \left.\left.\left.-\tilde{q}_{i}\right)-\rho_{\tau}\left(y_{i}-q_{i}\right)\right)\right] \\
& =\mathbb{S}_{n}\left[w_{i} \cdot\left(q_{i}-\tilde{q}_{i}\right) \cdot \Psi_{\tau}\left(y_{i}-q_{i}\right)\right]+\mathbb{S}_{n}\left[w_{i} \cdot W_{i}^{\natural}\left(\tilde{q}_{i}-q_{i}\right)\right] .
\end{aligned}
$$

Combining (41) and (42) with (40), we have

$$
\mathbb{S}_{n}\left[w_{i} \cdot\left(\tilde{q}_{i}-\hat{q}_{i}\right) \cdot \Psi_{\tau}\left(y_{i}-q_{i}\right)\right]-\mathbb{S}_{n}\left[w_{i} \cdot W_{i}^{\natural}\left(\tilde{q}_{i}-q_{i}\right)\right] \leq \lambda\left(\left\|b^{\star}\right\|_{1,2}-\|\widehat{b}\|_{1,2}\right)
$$

On the event $\mathcal{E}_{\mathrm{QR}}(\lambda)$,

$$
\mathbb{S}_{n}\left[w_{i} \cdot\left(\tilde{q}_{i}-\hat{q}_{i}\right) \cdot \Psi_{\tau}\left(y_{i}-q_{i}\right)\right] \geq-\frac{\lambda}{2}\left\|b^{\star}-\widehat{b}\right\|_{1,2} .
$$

Combining with the display above, we obtain that

$$
-\frac{\lambda}{2}\left\|b^{\star}-\widehat{b}\right\|_{1,2} \leq \mathbb{S}_{n}\left[w_{i} \cdot W_{i}^{\natural}\left(\tilde{q}_{i}-q_{i}\right)\right]+\lambda\left(\left\|b^{\star}\right\|_{1,2}-\|\widehat{b}\|_{1,2}\right) .
$$

Since

$$
\left\|b^{\star}\right\|_{1,2}-\|\widehat{b}\|_{1,2} \leq\left\|\left(b^{\star}-\widehat{b}\right)_{S^{\prime}}\right\|_{1,2}-\left\|\left(b^{\star}-\widehat{b}\right)_{N^{\prime}}\right\|_{1,2},
$$

we have

$$
\frac{\lambda}{2}\left\|\left(\widehat{b}-b^{\star}\right)_{N^{\prime}}\right\|_{1,2} \leq \mathbb{S}_{n}\left[w_{i} \cdot W_{i}^{\natural}\left(\tilde{q}_{i}-q_{i}\right)\right]+\frac{3 \lambda}{2}\left\|\left(\widehat{b}-b^{\star}\right)_{S^{\prime}}\right\|_{1,2},
$$

which completes the proof. 
Lemma 8. Under Assumption 6, for

$$
\lambda=4 \cdot\left(\max _{j \in[p]} \mathbb{S}_{n}\left[w_{i}^{2} x_{i j}^{2}\right]\right)^{1 / 2} \sqrt{\log (4 p / \gamma)}=O\left(\sqrt{\frac{\log p}{n h}}\right),
$$

we have

$$
\mathbb{P}\left\{\mathcal{E}_{\mathrm{QR}}(\lambda)\right\} \geq 1-\gamma
$$

Proof. We prove that

$$
\max _{j \in[p]}\left|\mathbb{S}_{n}\left[w_{i} \cdot x_{i j} \cdot \Psi_{\tau}\left(y_{i}-q_{i}\right)\right]\right| \leq \frac{\lambda}{2 \sqrt{2}}
$$

and

$$
\max _{j \in[p]}\left|\mathbb{S}_{n}\left[w_{i} \cdot x_{i j}\left(u_{i}-u\right) \cdot \Psi_{\tau}\left(y_{i}-q_{i}\right)\right]\right| \leq \frac{\lambda}{2 \sqrt{2}}
$$

Let

$$
Z_{i}=\frac{w_{i} \cdot x_{i j} \cdot \Psi_{\tau}\left(y_{i}-q_{i}\right)}{\sqrt{\mathbb{S}_{n}\left[w_{i}^{2} x_{i j}^{2}\right]}}
$$

and note that $\left|Z_{i}\right| \leq 1$ and $\mathbb{E}\left[Z_{i}\right]=0$. The Hoeffding's inequality (Theorem 2.8 Boucheron et al., 2013) gives us that

$$
\left|\mathbb{S}_{n}\left[Z_{i}\right]\right| \leq \sqrt{2 \log (2 / \gamma)}
$$

with probability $1-\gamma$. An application of the union bound gives us that

$$
\max _{j \in[p]} \mathbb{S}_{n}\left[w_{i} \cdot x_{i j} \cdot \Psi_{\tau}\left(y_{i}-q_{i}\right)\right] \leq\left(\max _{j \in[p]} \mathbb{S}_{n}\left[w_{i}^{2} x_{i j}^{2}\right]\right)^{1 / 2} \sqrt{2 \log (4 p / \gamma)}
$$

with probability $1-\gamma / 2$. This proves (45). Equation (46) is shown in the same way by noting that

$$
\mathbb{S}_{n}\left[w_{i}^{2} x_{i j}^{2}\left(u_{i}-u\right)^{2}\right] \leq \mathbb{S}_{n}\left[w_{i}^{2} x_{i j}^{2}\right] .
$$

Lemma 9. Let $\check{b}=b^{\star}+\delta$,

$$
\begin{aligned}
g_{i}(\delta)= & w_{i} \cdot\left(W_{i}^{\natural}\left(\Gamma_{i}^{\top} \check{b}-q_{i}\right)-W_{i}^{\natural}\left(\Gamma_{i}^{\top} b^{\star}-q_{i}\right)\right) \\
=w_{i} & \cdot\left(y_{i}-\Gamma_{i}^{\top} \check{b}\right)\left[\mathbb{1}\left\{\Gamma_{i}^{\top} \check{b} \leq y_{i}<q_{i}\right\}-\mathbb{1}\left\{q_{i}<y_{i}<\Gamma_{i}^{\top} \check{b}\right\}\right] \\
& \quad-w_{i} \cdot\left(y_{i}-\Gamma_{i}^{\top} b^{\star}\right)\left[\mathbb{1}\left\{\Gamma_{i}^{\top} b^{\star} \leq y_{i}<q_{i}\right\}-\mathbb{1}\left\{q_{i}<y_{i}<\Gamma_{i}^{\top} b^{\star}\right\}\right] .
\end{aligned}
$$

Then

$$
\sup _{\substack{\|\delta\|_{1,2} \leq R_{1} \\ \mathbb{S}_{n}\left[w_{i}\left(\Gamma_{i}^{\top} \delta\right)^{2}\right] \leq R_{2}}}\left|\left(\mathbb{S}_{n}-\mathbb{E} \mathbb{S}_{n}\right)\left[w_{i} \cdot g_{i}(\delta)\right]\right|
$$




$$
\begin{array}{r}
\lesssim R_{1} \sqrt{\frac{B_{X}^{2} B_{K}^{2}}{n h} \log (p)}+R_{1} \sqrt{\left(B_{w} B_{X} \sqrt{\frac{B_{X}^{2} B_{K}^{2}}{n h} \log (p)}+B_{w} \frac{R_{2}}{R_{1}}\right) \log (1 / \gamma)} \\
+R_{1} B_{w} B_{X} \log (1 / \gamma)
\end{array}
$$

with probability $1-\gamma$.

Proof. We will apply Lemma 25. Note that

$$
\begin{gathered}
\left|g_{i}(\delta)\right| \leq w_{i}\left(y_{i}-\Gamma_{i}^{\top} \check{b}\right) \cdot\left[\mathbb{1}\left\{\Gamma_{i}^{\top} \check{b} \leq y_{i}<q_{i}\right\}-\mathbb{1}\left\{q_{i}<y_{i}<\Gamma_{i}^{\top} \check{b}\right\}\right. \\
\left.-\mathbb{1}\left\{\Gamma_{i}^{\top} b^{\star} \leq y_{i}<q_{i}\right\}+\mathbb{1}\left\{q_{i}<y_{i}<\Gamma_{i}^{\top} b^{\star}\right\}\right] \\
+w_{i}\left(\Gamma_{i}^{\top} \check{b}-\Gamma_{i}^{\top} b^{\star}\right)\left[\mathbb{1}\left\{\Gamma_{i}^{\top} b^{\star} \leq y_{i}<q_{i}\right\}-\mathbb{1}\left\{q_{i}<y_{i}<\Gamma_{i}^{\top} b^{\star}\right\}\right] \\
\leq 2\left|w_{i} \Gamma_{i}^{\top} \delta\right| \leq 2 B_{w} B_{X} R_{1} .
\end{gathered}
$$

Therefore, $\left|g_{i}(\delta)-\mathbb{E}\left[g_{i}(\delta)\right]\right| \leq 4 B_{w} B_{X} R_{1}$. For the variance, we have

$$
\mathbb{E}\left[\sum_{i}\left(g_{i}(\delta)-\mathbb{E}\left[g_{i}(\delta)\right]\right)^{2}\right] \leq \mathbb{E}\left[\sum_{i} g_{i}^{2}(\delta)\right] \leq 4 \sum_{i \in[n]} w_{i}^{2}\left(\Gamma_{i}^{\top} \delta\right)^{2} \leq 4 B_{w} R_{2} .
$$

Finally, we bound the supremum of the process. We have

$$
\begin{aligned}
& \mathbb{E}\left[\sup _{\substack{\|\delta\|_{1,2} \leq R_{1} \\
\mathbb{S}_{n}\left[w_{i}\left(\Gamma_{i}^{\top} \delta\right)^{2}\right]=R_{2}}}\left|\left(\mathbb{S}_{n}-\mathbb{E S}_{n}\right)\left[g_{i}(\delta)\right]\right|\right] \\
& \stackrel{(i)}{\leq} 2 \mathbb{E}\left[\sup _{\substack{\|\delta\|_{1,2} \leq R_{1} \\
\mathbb{S}_{n}\left[w_{i}\left(\Gamma_{i}^{\top} \delta\right)^{2}\right]=R_{2}}}\left|\mathbb{S}_{n}\left[\epsilon_{i} \cdot g_{i}(\delta)\right]\right|\right] \\
& \stackrel{(i i)}{\leq} 4 \mathbb{E}\left[\sup _{\substack{\|\delta\|_{1,2} \leq R_{1} \\
\mathbb{S}_{n}\left[w_{i}\left(\Gamma_{i}^{\top} \delta\right)^{2}\right]=R_{2}}}\left|\mathbb{S}_{n}\left[\epsilon_{i} w_{i} \Gamma_{i}^{\top} \delta\right]\right|\right] \\
& \leq 4\|\delta\|_{1,2} \mathbb{E}\left[\max _{j \in[p]}\left|\mathbb{S}_{n}\left[\epsilon_{i} w_{i}\left\|\left[\begin{array}{c}
x_{i j} \\
x_{i j}\left(U_{i}-u\right)
\end{array}\right]\right\|_{2}\right]\right|\right] \\
& \stackrel{\text { (iii) }}{\leq} 8\|\delta\|_{1,2} \sqrt{\frac{B_{X}^{2} B_{K}^{2}}{n h} \log (2 p)},
\end{aligned}
$$

where $(i)$ follows from symmetrization (Lemma 11.4 Boucheron et al., 2013), (ii) from contraction inequality (Theorem 11.6 Boucheron et al., 2013), and (iii) from a maximum inequality for sub-Gaussian random variables (Theorem 2.5 Boucheron et al., 2013). The result now follows by plugging the pieces into Lemma 25. 
Lemma 10. Suppose Assumptions 3 and 6 hold. For a fixed $\delta$, we have

$$
\begin{aligned}
\mathbb{E S}_{n}\left[w_{i} \cdot\left(W_{i}\left(\Gamma_{i}^{\top}\left(b^{\star}+\delta\right)-q_{i}\right)-W_{i}\left(\Gamma_{i}^{\top} b^{\star}-q_{i}\right)\right)\right] \\
\quad \geq \frac{1}{3} \cdot\left(\underline{f} \mathbb{S}_{n}\left[w_{i} \cdot\left(\Gamma_{i}^{\top} \delta\right)^{2}\right] \wedge \kappa_{q} \cdot\left(\underline{f} \cdot \mathbb{S}_{n}\left[w_{i} \cdot\left(\Gamma_{i}^{\top} \delta\right)^{2}\right]\right)^{1 / 2}\right) .
\end{aligned}
$$

Proof. Using (33), we have

$$
\mathbb{E}\left[W_{i}^{\#}\left(\Gamma_{i}^{\top}\left(b^{\star}+\delta\right)-q_{i}\right)-W_{i}^{\#}\left(\Gamma_{i}^{\top} b^{\star}-q_{i}\right)\right]=-\left(\Gamma_{i}^{\top} \delta\right) \cdot \mathbb{E}\left[\psi_{\tau}\left(y_{i}-q_{i}\right)\right]=0
$$

and

$$
\begin{aligned}
\mathbb{E}\left[W_{i}^{\natural}\left(\Gamma_{i}^{\top}\left(b^{\star}+\delta\right)-q_{i}\right)-W_{i}^{\natural}\left(\Gamma_{i}^{\top} b^{\star}-q_{i}\right)\right] \\
\quad=\mathbb{E}\left[\int_{0}^{\Gamma_{i}^{\top} \delta}\left[\mathbb{1}\left\{y_{i} \leq \Gamma_{i}^{\top} b^{\star}+z\right\}-\mathbb{1}\left\{y_{i} \leq \Gamma_{i}^{\top} b^{\star}\right\}\right] d z\right] \\
=\int_{0}^{\Gamma_{i}^{\top} \delta}\left[F_{i}\left(\Gamma_{i}^{\top} b^{\star}+z\right)-F_{i}\left(\Gamma_{i}^{\top} b^{\star}\right)\right] d z \\
\quad=\int_{0}^{\Gamma_{i}^{\top} \delta}\left[z f_{i}\left(\Gamma_{i}^{\top} b^{\star}\right)+\frac{z^{2}}{2} f_{i}^{\prime}\left(\Gamma_{i}^{\top} b^{\star}+\tilde{z}\right)\right] d z \quad(\tilde{z} \in[0, z]) \\
\geq \frac{f}{2}\left(\Gamma_{i}^{\top} \delta\right)^{2}-\frac{\bar{f}^{\prime}}{6}\left(\Gamma_{i}^{\top} \delta\right)^{3} .
\end{aligned}
$$

Combining the last two displays, we obtain

$$
\begin{aligned}
\mathbb{E S}_{n}\left[w _ { i } \cdot \left(W _ { i } \left(\Gamma_{i}^{\top}\left(b^{\star}+\delta\right)\right.\right.\right. & \left.\left.\left.-q_{i}\right)-W_{i}\left(\Gamma_{i}^{\top} b^{\star}-q_{i}\right)\right)\right] \\
& \geq \frac{f}{2} \mathbb{S}_{n}\left[w_{i} \cdot\left(\Gamma_{i}^{\top} \delta\right)^{2}\right]-\frac{\bar{f}^{\prime}}{6} \mathbb{S}_{n}\left[w_{i} \cdot\left(\Gamma_{i}^{\top} \delta\right)^{3}\right] .
\end{aligned}
$$

We lower bound the above display in two cases. First, consider the case where

$$
\left(\underline{f} \cdot \mathbb{S}_{n}\left[w_{i} \cdot\left(\Gamma_{i}^{\top} \delta\right)^{2}\right]\right)^{1 / 2} \leq \kappa_{q} .
$$

From the definition of $\kappa_{q}$, we then obtain that

$$
\bar{f}^{\prime} \cdot \mathbb{S}_{n}\left[w_{i} \cdot\left(\Gamma_{i}^{\top} \delta\right)^{3}\right] \leq \underline{f} \cdot \mathbb{S}_{n}\left[w_{i} \cdot\left(\Gamma_{i}^{\top} \delta\right)^{2}\right]
$$

which combined with (47) gives us

$$
\begin{aligned}
\mathbb{E S}_{n}\left[w_{i} \cdot\left(W_{i}\left(\Gamma_{i}^{\top}\left(b^{\star}+\delta\right)-q_{i}\right)-W_{i}\left(\Gamma_{i}^{\top} b^{\star}-q_{i}\right)\right)\right] & \\
& \geq \frac{f}{3} \mathbb{S}_{n}\left[w_{i} \cdot\left(\Gamma_{i}^{\top} \delta\right)^{2}\right] .
\end{aligned}
$$

Next, we consider the case where

$$
\left(\underline{f} \cdot \mathbb{S}_{n}\left[w_{i} \cdot\left(\Gamma_{i}^{\top} \delta\right)^{2}\right]\right)^{1 / 2}>\kappa_{q} .
$$


Let $\bar{b}=b^{\star}+(1-\alpha) \delta$ for some $\alpha \in(0,1)$ to be determined later. Using the convexity of $\rho_{\tau}(\cdot)$, we have that

$$
\begin{aligned}
\mathbb{E S}_{n}\left[w_{i} \cdot\left(W_{i}\left(\Gamma_{i}^{\top}\left(b^{\star}+\delta\right)-q_{i}\right)-W_{i}\left(\Gamma_{i}^{\top} b^{\star}-q_{i}\right)\right)\right] \\
\quad \geq \frac{1}{1-\alpha}\left(\mathbb{E} \mathbb{S}_{n}\left[w_{i} \cdot\left(W_{i}\left(\Gamma_{i}^{\top} \bar{b}-q_{i}\right)-W_{i}\left(\tilde{q}_{i}-q_{i}\right)\right)\right]\right) \\
\quad \geq \frac{1}{1-\alpha}\left(\frac{f}{2} \mathbb{S}_{n}\left[w_{i} \cdot\left(\Gamma_{i}^{\top}\left(\bar{b}-b^{\star}\right)\right)^{2}\right]-\frac{\bar{f}^{\prime}}{6} \mathbb{S}_{n}\left[w_{i} \cdot\left(\Gamma_{i}^{\top}\left(\bar{b}-b^{\star}\right)\right)^{3}\right]\right),
\end{aligned}
$$

where the second inequality follows from (47). We want to choose $\alpha$ such that

$$
\underline{f} \cdot \mathbb{S}_{n}\left[w_{i} \cdot\left(\Gamma_{i}^{\top}\left(\bar{b}-b^{\star}\right)\right)^{2}\right]=\bar{f}^{\prime} \mathbb{S}_{n}\left[w_{i} \cdot\left(\Gamma_{i}^{\top}\left(\bar{b}-b^{\star}\right)\right)^{3}\right],
$$

which leads to

$$
1-\alpha=\frac{f \cdot \mathbb{S}_{n}\left[w_{i} \cdot\left(\Gamma_{i}^{\top} \delta\right)^{2}\right]}{\bar{f}^{\prime} \mathbb{S}_{n}\left[w_{i} \cdot\left(\Gamma_{i}^{\top} \delta\right)^{3}\right]} .
$$

Combining with (49), we have

$$
\begin{aligned}
\mathbb{E S}_{n} & {\left[w_{i} \cdot\left(W_{i}\left(\Gamma_{i}^{\top}\left(b^{\star}+\delta\right)-q_{i}\right)-W_{i}\left(\Gamma_{i}^{\top} b^{\star}-q_{i}\right)\right)\right] } \\
\geq & \frac{1}{3} \cdot \frac{\left(\underline{f} \cdot \mathbb{S}_{n}\left[w_{i} \cdot\left(\Gamma_{i}^{\top} \delta\right)^{2}\right]\right)^{2}}{\bar{f}^{\prime} \mathbb{S}_{n}\left[w_{i} \cdot\left(\Gamma_{i}^{\top} \delta\right)^{3}\right]} \\
\geq & \frac{\kappa_{q}}{3} \cdot\left(\underline{f} \cdot \mathbb{S}_{n}\left[w_{i} \cdot\left(\Gamma_{i}^{\top} \delta\right)^{2}\right]\right)^{1 / 2} .
\end{aligned}
$$

The proof follows by combining the lower bounds in (48) and (50).

Lemma 11. Under our model assumptions,

$$
\begin{aligned}
& \mathbb{S}_{n}\left[w_{i} \cdot W_{i}^{\natural}\left(\tilde{q}_{i}-q_{i}\right)\right] \\
& \quad \leq 2 \sqrt{2 \log (2 / \gamma)} \cdot\left(\bar{f} \cdot \mathbb{S}_{n}\left[w_{i} \cdot\left(\tilde{q}_{i}-q_{i}\right)^{2}\right]+\sqrt{\mathbb{S}_{n}\left[w_{i}^{2}\left(\tilde{q}_{i}-q_{i}\right)^{2}\right]}\right)
\end{aligned}
$$

holds with probability $1-\gamma$.

Proof. We will prove the lemma using Theorem 2.16 of de la Peña et al. (2009). Note that $W_{i}^{\natural}\left(\tilde{q}_{i}-q_{i}\right)$ is positive and

$$
\begin{aligned}
\mathbb{E}\left[W_{i}^{\natural}\left(\tilde{q}_{i}-q_{i}\right)\right] & =\int_{0}^{\tilde{q}_{i}-q_{i}}\left[F_{i}\left(q_{i}+z\right)-F_{i}\left(q_{i}\right)\right] d z \\
& =\int_{0}^{\tilde{q}_{i}-q_{i}} f_{i}\left(\tilde{z}_{i}\right) z d z \leq \frac{\bar{f}}{2}\left(\tilde{q}_{i}-q_{i}\right)^{2},
\end{aligned}
$$


where $\tilde{z}_{i}$ is a point between 0 and $z$. Therefore, the Markov's inequality gives us

$$
\mathbb{P}\left\{\mathbb{S}_{n}\left[w_{i} \cdot W_{i}^{\natural}\left(\tilde{q}_{i}-q_{i}\right)\right] \geq 2 \bar{f} \cdot \mathbb{S}_{n}\left[w_{i} \cdot\left(\tilde{q}_{i}-q_{i}\right)^{2}\right]\right\} \leq \frac{1}{4} .
$$

Furthermore, since $\left|W_{i}^{\natural}\left(\tilde{q}_{i}-q_{i}\right)\right| \leq\left|\tilde{q}_{i}-q_{i}\right|$, we have that

$$
\mathbb{P}\left\{\mathbb{S}_{n}\left[\left(w_{i} \cdot W_{i}^{\natural}\left(\tilde{q}_{i}-q_{i}\right)\right)^{2}\right] \geq \mathbb{S}_{n}\left[w_{i}^{2}\left(\tilde{q}_{i}-q_{i}\right)^{2}\right]\right\}=0 \leq \frac{1}{4} .
$$

Invoking Theorem 2.16 of de la Peña et al. (2009), define

$$
\begin{array}{cc}
a=2 \bar{f} \mathbb{S}_{n}\left[w_{i} \cdot\left(\tilde{q}_{i}-q_{i}\right)^{2}\right], & b=\sqrt{\mathbb{S}_{n}\left[w_{i}^{2}\left(\tilde{q}_{i}-q_{i}\right)^{2}\right]}, \\
S_{n}=\mathbb{S}_{n}\left[w_{i} \cdot W_{i}^{\natural}\left(\tilde{q}_{i}-q_{i}\right)\right], & V_{n}^{2}=\mathbb{S}_{n}\left[\left(w_{i} \cdot W_{i}^{\natural}\left(\tilde{q}_{i}-q_{i}\right)\right)^{2}\right],
\end{array}
$$

and observe that $V_{n} \leq b^{2}$, we obtain that

$$
\mathbb{P}\left\{S_{n} \geq x\left(a+b+V_{n}\right)\right\} \leq 2 e^{\frac{-x^{2}}{2}},
$$

which completes the proof.

Lemma 12. Under Assumptions 1, 2, 4, and 6, we have that

$$
\sum_{i} w_{i}\left(\tilde{q}_{i}-q_{i}\right)^{2} \leq 2 h^{4} s B_{X}^{2} B_{\beta} B_{K}+2 \frac{\epsilon_{R}^{2}}{\underline{f}^{2}}=O\left(h^{4}+\epsilon_{R}^{2}\right)
$$

and

$$
\sum_{i} w_{i}^{2}\left(\tilde{q}_{i}-q_{i}\right)^{2} \leq B_{w} \cdot\left(2 h^{4} s B_{X}^{2} B_{\beta} B_{K}+2 \frac{\epsilon_{R}^{2}}{f^{2}}\right)=O\left(\frac{h^{3}}{n}+\frac{\epsilon_{R}^{2}}{n h}\right) .
$$

Proof. First, the assumption on the density $f_{i}$ proves that

$$
\left|x_{i}^{\top} \beta^{\star}\left(\tau, u_{i}\right)-q_{i}\right| \leq \frac{R_{i}}{\underline{f}} .
$$

Then

$$
\begin{aligned}
& \sum_{i} w_{i}\left(\tilde{q}_{i}-q_{i}\right)^{2} \\
& \left.\quad \leq 2 \sum_{i} w_{i}\left(\tilde{q}_{i}-x_{i}^{\top} \beta^{\star}\left(\tau, u_{i}\right)\right)\right)^{2}+2 \sum_{i} w_{i}\left(x_{i}^{\top} \beta^{\star}\left(\tau, u_{i}\right)-q_{i}\right)^{2} \\
& \quad \leq 2 \sum_{i} w_{i}\left[x_{i}^{\top}\left(\beta^{\star}\left(\tau, u_{i}\right)-\beta^{\star}(\tau, u)-\left(u_{i}-u\right) \cdot \nabla_{u} \beta^{\star}(\tau, u)\right)\right]^{2}+2 \sum_{i} w_{i} \frac{R_{i}^{2}}{f^{2}} \\
& \quad \leq 2 \sum_{i} w_{i}\left\|x_{i}\right\|_{\infty}^{2}\left\|\beta^{\star}\left(\tau, u_{i}\right)-\beta^{\star}(\tau, u)-\left(u_{i}-u\right) \cdot \nabla_{u} \beta^{\star}(\tau, u)\right\|_{1}^{2}+2 \frac{\epsilon_{R}^{2}}{f^{2}}
\end{aligned}
$$




$$
\begin{aligned}
& \leq 2 s B_{X}^{2} B_{\beta} \sum_{i} w_{i}\left(u_{i}-u\right)^{4}+2 \frac{\epsilon_{R}^{2}}{\underline{f}^{2}} \\
& =h^{4} \cdot 2 s B_{X}^{2} B_{\beta} \sum_{i} w_{i}\left(\frac{u_{i}-u}{h}\right)^{4}+2 \frac{\epsilon_{R}^{2}}{\underline{f}^{2}} \\
& \leq 2 h^{4} s B_{X}^{2} B_{\beta} B_{K}+2 \frac{\epsilon_{R}^{2}}{\underline{f}^{2}}
\end{aligned}
$$

which proves the first statement.

The second statement immediately follows since

$$
\sum_{i} w_{i}^{2}\left(\tilde{q}_{i}-q_{i}\right)^{2} \leq\|w\|_{\infty} \cdot \sum_{i} w_{i}\left(\tilde{q}_{i}-q_{i}\right)^{2} \leq 2 h^{4} s B_{w} B_{X}^{2} B_{\beta} B_{K}+2 B_{w} \frac{\epsilon_{R}^{2}}{\underline{f}^{2}}
$$

\section{A.6. Proof of Theorem 1}

Throughout the section, use $\widehat{b}^{\lambda}$ to denote $\widehat{b}$ defined in Section 3. In particular, $\widehat{b}$ is defined in (35), $\widehat{b}^{\lambda}$ is $\widehat{b}$ thresholded at level $\lambda$, i.e., $\widehat{b}_{j}^{\lambda}=\widehat{b}_{j} \cdot \mathbb{1}\left\{\widehat{b}_{j}^{2}+\widehat{b}_{j+p}^{2}>\lambda^{2}\right\}$, $1 \leq j \leq p$, and $\widehat{b}_{j}^{\lambda}=\widehat{b}_{j} \cdot \mathbb{1}\left\{\widehat{b}_{j}^{2}+\widehat{b}_{j-p}^{2}>\lambda^{2}\right\}, p+1 \leq j \leq 2 p$.

Let $S^{\prime}=\operatorname{support}\left(b^{\star}\right)$. By Assumption $4,\left|S^{\prime}\right| \leq c s$ for some absolute constant c. Therefore,

$$
\begin{aligned}
\left\|\widehat{b}^{\lambda}-b^{\star}\right\|_{1,2} & \leq\left\|\left(\widehat{b}^{\lambda}-b^{\star}\right)_{S^{\prime}}\right\|_{1,2}+\left\|\left(\widehat{b}^{\lambda}\right)_{S^{\prime}}\right\|_{1,2} \\
& \leq\left\|\left(\widehat{b}^{\lambda}-\widehat{b}\right)_{S^{\prime}}\right\|_{1,2}+\left\|\left(\widehat{b}-b^{\star}\right)_{S^{\prime}}\right\|_{1,2}+\left\|\left(\widehat{b}^{\lambda}\right)_{S^{\prime}}\right\|_{1,2} \\
& \leq c s \lambda+\left\|\left(\widehat{b}-b^{\star}\right)_{S^{\prime}}\right\|_{1,2}+\left\|\left(\widehat{b}^{\lambda}\right)_{S^{\prime}}\right\|_{1,2} \\
& \leq c s \lambda+\left\|\widehat{b}-b^{\star}\right\|_{1,2},
\end{aligned}
$$

where the third inequality comes from the definition of $\widehat{b}^{\lambda}$. Furthermore, notice that $\left\|\widehat{b}^{\lambda}-b^{\star}\right\|_{1,2} \geq\left(\left\|\widehat{b}^{\lambda}\right\|_{0,2}-\left|S^{\prime}\right|\right) \lambda$. Therefore we have

$$
\left\|\widehat{b}-b^{\star}\right\|_{1,2} \geq\left[\left\|\widehat{b}^{\lambda}\right\|_{0,2}-2 c s\right] \lambda .
$$

Therefore, $\left\|\widehat{b}^{\lambda}\right\|_{0,2} \leq 2 c s+\left\|\widehat{b}-b^{\star}\right\|_{1,2} / \lambda$.

Because $\lambda=O\left(\sqrt{\frac{\log (n p)}{n h}}\right)$ and $\left\|\widehat{b}-b^{\star}\right\|_{1,2}=O_{p}\left(s \sqrt{\frac{\log (n p)}{n h}}\right)$ from Theorem 4, $\left\|\widehat{b}^{\lambda}\right\|_{0,2} \leq O_{p}(s)$. Now we have shown (23) and (24).

To show (22), we first use the triangle inequality,

$$
\begin{aligned}
\sqrt{\mathbb{S}_{n}\left[w_{i} \cdot\left(\Gamma_{i}^{\top}\left(\widehat{b}^{\lambda}-b^{\star}\right)\right)^{2}\right]} \\
\quad \leq \sqrt{\mathbb{S}_{n}\left[w_{i} \cdot\left(\Gamma_{i}^{\top}\left(\widehat{b}^{\lambda}-\widehat{b}\right)\right)^{2}\right]}+\sqrt{\mathbb{S}_{n}\left[w_{i} \cdot\left(\Gamma_{i}^{\top}\left(\widehat{b}-b^{\star}\right)\right)^{2}\right]}
\end{aligned}
$$


Without loss of generality, we can order the components so that $\left(\widehat{b}_{j}^{\lambda}-\widehat{b}_{j}\right)^{2}+$ $\left(\widehat{b}_{j+p}^{\lambda}-\widehat{b}_{j+p}\right)^{2}$ is decreasing. Let $T_{1}$ be the set of $c s$ indices corresponding to the largest values of $\left(\widehat{b}_{j}^{\lambda}-\widehat{b}_{j}\right)^{2}+\left(\widehat{b}_{j+p}^{\lambda}-\widehat{b}_{j+p}\right)^{2}$, similarly, let $T_{k}$ be the set of $c s$ indices corresponding to the largest values of $\left(\widehat{b}_{j}^{\lambda}-\widehat{b}_{j}\right)^{2}+\left(\widehat{b}_{j+p}^{\lambda}-\widehat{b}_{j+p}\right)^{2}$ outside $\cup_{m=1}^{k-1} T_{m}$. By monotonicity, $\left\|\left(\widehat{b}^{\lambda}-\widehat{b}\right)_{T_{k}}\right\|_{2} \leq\left\|\left(\widehat{b}^{\lambda}-\widehat{b}\right)_{T_{k-1}}\right\|_{1,2} / \sqrt{c s}$. Then we have,

$$
\begin{aligned}
& \sqrt{\mathbb{S}_{n}\left[w_{i} \cdot\left(\Gamma_{i}^{\top}\left(\widehat{b}^{\lambda}-\widehat{b}\right)\right)^{2}\right]} \\
& =\sqrt{\sum_{k=1}^{\left\lceil\frac{p}{c s}\right\rceil} \mathbb{S}_{n}\left[w_{i}\left(\Gamma_{i}^{\top}\left(\widehat{b}^{\lambda}-\widehat{b}\right)_{T_{k}}\right)^{2}\right]} \\
& \leq \sqrt{\mathbb{S}_{n}\left[w_{i} \cdot\left(\Gamma_{i}^{\top}\left(\widehat{b}^{\lambda}-\widehat{b}\right)_{T_{1}}\right)^{2}\right]}+\sqrt{\sum_{k=2}^{\left\lceil\frac{p}{c s}\right\rceil} \mathbb{S}_{n}\left[w_{i}\left(\Gamma_{i}^{\top}\left(\widehat{b}^{\lambda}-\widehat{b}\right)_{T_{k}}\right)^{2}\right]} \\
& \leq \kappa_{+}\left\|\left(\widehat{b}^{\lambda}-\widehat{b}\right)_{T_{1}}\right\|_{2}+\kappa_{+} \sum_{k=2}^{\left\lceil\frac{p}{c s}\right\rceil}\left\|\left(\widehat{b}^{\lambda}-\widehat{b}\right)_{T_{k}}\right\|_{2} \\
& \leq \kappa_{+}\left\|\left(\widehat{b}^{\lambda}-\widehat{b}\right)_{T_{1}}\right\|_{2}+\kappa_{+} \sum_{k=1}^{\left\lceil\frac{p}{c s}\right\rceil}\left\|\left(\widehat{b}^{\lambda}-\widehat{b}\right)_{T_{k}}\right\|_{1,2} / \sqrt{c s} \\
& \leq \kappa_{+} \lambda+\kappa_{+}\left\|\left(\widehat{b}^{\lambda}-\widehat{b}\right)\right\|_{1,2} / \sqrt{c s} \\
& =O_{p}\left(\sqrt{\left.\frac{s \log (n p)}{n h}\right) .}\right.
\end{aligned}
$$

In addition, from Theorem $4, \mathbb{S}_{n}\left[w_{i} \cdot\left(\Gamma_{i}^{\top}\left(\widehat{b}-b^{\star}\right)\right)^{2}\right] \leq O_{p}\left(\frac{s \log (n p)}{n h}\right)$. Therefore, the first inequality holds.

\section{A.7. Proof of Theorem 2}

Our starting point is the basic inequality

$$
\begin{aligned}
\lambda_{V} & \left(\left\|V^{\star}\right\|_{1, F}-\|\widehat{V}\|_{1, F}\right) \\
& \geq \operatorname{trace}\left(\frac{1}{2} \delta_{v}^{\top} \widehat{H}\left(\delta_{b}\right) \delta_{v}+\delta_{v}^{\top}\left(\widehat{H}\left(\delta_{b}\right)-H^{\star}\right) V^{\star}+\delta_{v}^{\top}\left(H^{\star} V^{\star}-E_{a}\right)\right),
\end{aligned}
$$

where $\delta_{b}=\widehat{b}-b^{\star}$ and $\delta_{v}=\widehat{V}-V^{\star}$. The above display can be rearranged as

$$
\operatorname{trace}\left(\frac{1}{2} \delta_{v}^{\top} \widehat{H}\left(\delta_{b}\right) \delta_{v}\right)
$$




$$
\begin{aligned}
& \leq \lambda_{V}\left(\left\|V^{\star}\right\|_{1, F}-\|\widehat{V}\|_{1, F}\right)-\operatorname{trace}( \delta_{v}^{\top} \\
&\left.\left(\widehat{H}\left(\delta_{b}\right)-H^{\star}\right) V^{\star}\right) \\
&-\operatorname{trace}\left(\delta_{v}^{\top}\left(H^{\star} V^{\star}-E_{a}\right)\right) .
\end{aligned}
$$

Denote

$$
\begin{aligned}
D:=B_{A} & \left(\sqrt{\frac{\bar{f} \cdot \log (p / \gamma)}{n h h_{f}}}\right. \\
& \left.+\bar{f}^{\prime}\left(2 B_{K} h_{f}+2 B_{K}\left(h^{4} s B_{X} B_{\beta}+\frac{\epsilon_{R}^{2}}{\underline{f}^{2}}\right)^{\frac{1}{2}}+s \sqrt{\frac{B_{K} \log (n p)}{n h}}\right)\right),
\end{aligned}
$$

where $B_{A}$ is defined in Lemma 13 . By Lemma 13, with probability at least $1-2 \gamma$,

$$
\left|\operatorname{trace}\left(\delta_{v}^{\top}\left(\widehat{H}\left(\delta_{b}\right)-H^{\star}\right) V^{\star}\right)\right| \leq\left\|\delta_{v}\right\|_{F} \cdot D .
$$

By assumption 5 ,

$$
\left|\operatorname{trace}\left(\delta_{v}^{\top}\left(H^{\star} V^{\star}-E_{a}\right)\right)\right| \leq\left\|\delta_{v}\right\|_{1, F}\left\|H^{\star} V^{\star}-E_{a}\right\|_{\infty, F} \leq \lambda^{\star}\left\|\delta_{v}\right\|_{1, F} .
$$

Since $\left\|V^{\star}\right\|_{1, F}-\|\widehat{V}\|_{1, F} \leq\left\|\left(\delta_{v}\right)_{S}\right\|_{1, F}-\left\|\left(\delta_{v}\right)_{N}\right\|_{1, F}$, we have

$$
\begin{aligned}
& \lambda_{V}\left\|\left(\delta_{v}\right)_{N}\right\|_{1, F} \\
& \leq \lambda_{V}\left\|\left(\delta_{v}\right)_{S}\right\|_{1, F}+\left|\operatorname{trace}\left(\delta_{v}^{\top}\left(\widehat{H}\left(\delta_{b}\right)-H^{\star}\right) V^{\star}\right)\right|+\left|\operatorname{trace}\left(\delta_{v}^{\top}\left(H^{\star} V^{\star}-E_{a}\right)\right)\right| \\
& \leq \lambda_{V}\left\|\left(\delta_{v}\right)_{S}\right\|_{1, F}+\left\|\delta_{v}\right\|_{F} \cdot D+\lambda^{\star}\left\|\delta_{v}\right\|_{1, F} \\
& \leq \lambda_{V}\left\|\left(\delta_{v}\right)_{S}\right\|_{1, F}+\left\|\delta_{v}\right\|_{F} \cdot D+\frac{\lambda_{V}}{2}\left\|\delta_{v}\right\|_{1, F} .
\end{aligned}
$$

Therefore,

$$
\frac{\lambda_{V}}{2}\left\|\left(\delta_{v}\right)_{N}\right\|_{1, F} \leq \frac{3 \lambda_{V}}{2}\left\|\left(\delta_{v}\right)_{S}\right\|_{1, F}+\left\|\delta_{v}\right\|_{F} \cdot D
$$

We consider two cases according to whether $\frac{3 \lambda_{V}}{2}\left\|\left(\delta_{v}\right)_{S}\right\|_{1, F} \geq\left\|\delta_{v}\right\|_{F} \cdot D$ or not. If

$$
\frac{3 \lambda_{V}}{2}\left\|\left(\delta_{v}\right)_{S}\right\|_{1, F} \geq\left\|\delta_{v}\right\|_{F} \cdot D
$$

then

$$
\left\|\left(\delta_{v}\right)_{N}\right\|_{1, F} \leq 6\left\|\left(\delta_{v}\right)_{S}\right\|_{1, F} .
$$

Therefore, we have

$$
\left\|\widehat{V}-V^{\star}\right\|_{1, F} \leq 7\left\|\left(\widehat{V}-V^{\star}\right)_{S}\right\|_{1, F} \leq 7 \sqrt{s_{2}}\left\|\left(\widehat{V}-V^{\star}\right)_{S}\right\|_{F} \leq 7 \sqrt{s_{2}}\left\|\delta_{v}\right\|_{F} .
$$

On the other hand, from the basic inequality (52),

$$
\lambda_{V}\left\|\widehat{V}-V^{\star}\right\|_{1, F}
$$




$$
\begin{aligned}
& \geq \operatorname{trace}\left(\frac{1}{2} \delta_{v}^{\top} \widehat{H}\left(\delta_{b}\right) \delta_{v}+\delta_{v}^{\top}\left(\widehat{H}\left(\delta_{b}\right)-H^{\star}\right) V^{\star}+\delta_{v}^{\top}\left(H^{\star} V^{\star}-E_{a}\right)\right) \\
& \geq \frac{f \kappa_{-}^{2}}{2}\left\|\delta_{v}\right\|_{F}^{2}-o_{p}(1)\left(\left\|\delta_{v}\right\|_{F}+\frac{\left\|\delta_{v}\right\|_{1, F}}{\sqrt{s_{2}}}\right)^{2}-\frac{3 \lambda_{V}}{2}\left\|\left(\delta_{v}\right)_{S}\right\|_{1, F}-\lambda^{\star}\left\|\delta_{v}\right\|_{1, F},
\end{aligned}
$$

where the second inequality above is because $\delta_{v} \in \mathbb{C}\left(S_{2}\right)$. Therefore, Assumption 6 holds and we can apply Lemma 18. Because $\lambda_{V} \geq 2 \lambda^{\star}$, after rearrangement and combining with (54), we get

$$
\left\|\delta_{v}\right\|_{F} \leq \frac{24 \lambda_{V} \sqrt{s_{2}}}{\underline{f} \kappa_{-}^{2}-o_{p}(1)}=O_{p}\left(\sqrt{\frac{s \log p}{n h h_{f}}}\right)
$$

and

$$
\left\|\delta_{v}\right\|_{1, F} \leq 7 \sqrt{s_{2}}\left\|\delta_{v}\right\|_{F}=O_{p}\left(s \sqrt{\frac{\log p}{n h h_{f}}}\right) .
$$

On the other hand, if

$$
\frac{3 \lambda_{V}}{2}\left\|\left(\delta_{v}\right)_{S}\right\|_{1, F} \leq\left\|\delta_{v}\right\|_{F} \cdot D
$$

then, from (53), we have

$$
\frac{\lambda_{V}}{2}\left\|\left(\delta_{v}\right)_{N}\right\|_{1, F} \leq 2\left\|\delta_{v}\right\|_{F} \cdot D
$$

Therefore

$$
\frac{\lambda_{V}}{2}\left\|\left(\delta_{v}\right)_{N}\right\|_{1, F} \leq 2 D\left\|\delta_{v}\right\|_{F} \leq 2 D\left\|\delta_{v}\right\|_{1, F}=2 D\left(\left\|\left(\delta_{v}\right)_{N}\right\|_{1, F}+\left\|\left(\delta_{v}\right)_{S}\right\|_{1, F}\right),
$$

which implies

$$
\left\|\left(\delta_{v}\right)_{N}\right\|_{1, F} \leq \frac{\frac{4 D}{\lambda_{V}}}{1-\frac{4 D}{\lambda_{V}}}\left\|\left(\delta_{v}\right)_{S}\right\|_{1, F} .
$$

From Assumption 5, we can see that $B_{A} \lesssim B_{X} B_{V}$. Then under Assumption 7, $h \asymp n^{-1 / 3}$ and $h_{f} \asymp n^{-1 / 3}$, and by Assumption $4, \epsilon_{R} \asymp \sqrt{\frac{\log n p}{n h}}$, we have

$$
D \asymp \lambda^{\star} \asymp B_{V} \sqrt{\frac{\log p}{n h h_{f}}} .
$$

Therefore, there exists $\lambda_{V}>2 \lambda^{\star}$ so that $\frac{4 D}{\lambda_{V}} \leq \frac{6}{7}$. With such a choice of $\lambda_{V}$, $\delta_{v} \in \mathbb{C}(S)$. Therefore, from (55) and (56),

$$
\lambda_{V}\left\|\delta_{v}\right\|_{1, F} \leq \frac{14}{3}\left\|\delta_{v}\right\|_{F} D .
$$


On the other hand, by applying Lemma 18 to the basic inequality (52), we have

$$
\lambda_{V}\left\|\delta_{v}\right\|_{1, F} \geq \underline{f} \kappa_{-}^{2}\left\|\delta_{v}\right\|_{F}^{2}-o_{p}(1)\left(\left\|\delta_{v}\right\|_{F}+\frac{\left\|\delta_{v}\right\|_{1, F}}{\sqrt{s_{2}}}\right)^{2}-2\left\|\delta_{v}\right\|_{F} D .
$$

Combining the two we have

$$
\frac{20}{3} D\left\|\delta_{v}\right\|_{F} \geq \underline{f} \kappa_{-}^{2}\left\|\delta_{v}\right\|_{F}^{2}-o_{p}(1)\left(\left\|\delta_{v}\right\|_{F}+\frac{14 D}{3 \lambda_{V} \sqrt{s_{2}}}\left\|\delta_{v}\right\|_{F}\right)^{2} .
$$

Because $D \asymp \lambda_{V}=O\left(B_{V} \sqrt{\frac{\log p}{n h h_{f}}}\right)$, we have

$$
\left\|\delta_{v}\right\|_{F} \leq \frac{20}{3} \frac{D}{\underline{f} \kappa_{-}^{2}-o_{p}(1)}=O_{p}\left(B_{V} \sqrt{\frac{s \log p}{n h h_{f}}}\right)
$$

and

$$
\left\|\delta_{v}\right\|_{1, F} \leq \frac{14 D}{3 \lambda_{V}} \sqrt{s_{2}}\left\|\delta_{v}\right\|_{F}=O_{p}\left(s B_{V} \sqrt{\frac{\log p}{n h h_{f}}}\right) .
$$

To complete the proof, we need to establish a few technical lemmas next.

Lemma 13. Suppose that the growth conditions in Assumption 7 is satisfied, and $r_{b} \asymp s \sqrt{\frac{\log (n p)}{n h}}$. Define $B_{A}=\max _{l}\left\|A_{i l}\right\|_{F}$ and $A_{i l}=\Gamma_{i l} \Gamma_{i}^{\top} V^{\star}$ where $l \in[p]$ and $\Gamma_{i l}=\left(\Gamma_{i, l}, \Gamma_{i, l+p}\right)^{\top}$. For any $\gamma>0$ such that $\bar{f} \geq\left(n h h_{f}\right)^{-1} \log (p / \gamma)$ and $r_{b}=O\left(h_{f} \log (p / \gamma) / B_{X}\right)$, we have

$$
\begin{aligned}
\max _{l \in[p]} \sup _{\substack{\left\|\delta_{b}\right\|_{0,2} \leq m \\
\left\|\delta_{b}\right\|_{1,2} \leq r_{b}}}\left\|\mathbb{S}_{n}\left[w_{i}\left(\hat{f}_{i}\left(\delta_{b}\right)-f_{i}\left(\tilde{q}_{i}\right)\right) \Gamma_{i l} \Gamma_{i}^{\top} V^{\star}\right]\right\|_{F} \\
\leq B_{A}\left(\sqrt{\frac{\bar{f} \cdot \log (p / \gamma)}{n h h_{f}}}\right. \\
\left.+\bar{f}^{\prime}\left(2 B_{K} h_{f}+2 B_{K}\left(h^{4} s B_{X} B_{\beta}+\frac{\epsilon_{R}^{2}}{\underline{f}^{2}}\right)^{1 / 2}+s \sqrt{\frac{\log (n p)}{n h}} \sqrt{B_{K}}\right)\right)
\end{aligned}
$$

with probability $1-2 \gamma$.

Proof. We have

$$
\begin{aligned}
& \max _{l \in[p]} \sup _{\substack{\left\|\delta_{b}\right\|_{0,2} \leq m \\
\left\|\delta_{b}\right\|_{1,2} \leq r_{b}}}\left\|\mathbb{S}_{n}\left[w_{i}\left(\hat{f}_{i}\left(\delta_{b}\right)-f_{i}\left(\tilde{q}_{i}\right)\right) \Gamma_{i l} \Gamma_{i}^{\top} V^{\star}\right]\right\|_{F} \\
& \leq \max _{l \in[p]} \sup _{\substack{\left\|\delta_{\delta}\right\|_{0,2} \leq m \\
\left\|\delta_{b}\right\|_{1,2} \leq r_{b}}}\left[\left\|\left(\mathbb{S}_{n}-\mathbb{E} \mathbb{S}_{n}\right)\left[w_{i}\left(\hat{f}_{i}\left(\delta_{b}\right)-\hat{f}_{i}(0)\right) \cdot A_{i l}\right]\right\|_{F}\right. \\
& +\left\|\left(\mathbb{S}_{n}-\mathbb{E} \mathbb{S}_{n}\right)\left[w_{i} \cdot\left(\hat{f}_{i}(0)-f_{i}\left(\tilde{q}_{i}\right)\right) \cdot A_{i l}\right]\right\|_{F}
\end{aligned}
$$




$$
\begin{aligned}
& \left.+\left\|\mathbb{E}_{n}\left[w_{i}\left(\hat{f}_{i}\left(\delta_{b}\right)-\hat{f}_{i}(0)\right) \Gamma_{i l} \Gamma_{i}^{\top} V^{\star}\right]\right\|_{F}\right] \\
& \left.+\left\|\mathbb{E}_{n}\left[w_{i}\left(\hat{f}_{i}(0)-f_{i}\left(\tilde{q}_{i}\right)\right) \Gamma_{i l} \Gamma_{i}^{\top} V^{\star}\right]\right\|_{F}\right] \\
& \leq \max _{l \in[p]} \sup _{\substack{\left\|\delta_{b}\right\|_{0,2} \leq m \\
\left\|\delta_{b}\right\|_{1,2} \leq r_{b}}}\left[\left\|\left(\mathbb{S}_{n}-\mathbb{E S}_{n}\right)\left[w_{i}\left(\hat{f}_{i}\left(\delta_{b}\right)-\hat{f}_{i}(0)\right) \cdot A_{i l}\right]\right\|_{F}\right. \\
& \left.+\left\|\left(\mathbb{S}_{n}-\mathbb{E S}_{n}\right)\left[w_{i} \cdot \hat{f}_{i}(0) \cdot A_{i l}\right]\right\|_{F}+\left\|\mathbb{E S}_{n}\left[w_{i}\left(\hat{f}_{i}\left(\delta_{b}\right)-\hat{f}_{i}(0)\right) \Gamma_{i l} \Gamma_{i}^{\top} V^{\star}\right]\right\|_{F}\right] \\
& \left.+\left\|\mathbb{E S}_{n}\left[w_{i}\left(\hat{f}_{i}(0)-f_{i}\left(\tilde{q}_{i}\right)\right) \Gamma_{i l} \Gamma_{i}^{\top} V^{\star}\right]\right\|_{F}\right] \\
& \leq B_{A}\left(\sqrt{\frac{\bar{f} \cdot \log (p / \gamma)}{n h h_{f}}}\right. \\
& \left.\quad+\bar{f}^{\prime}\left(B_{K} h_{f}+2 B_{K}\left(h^{4} s B_{X} B_{\beta}+\frac{\epsilon_{R}^{2}}{f^{2}}\right)^{1 / 2}+s \sqrt{\frac{\log (n p)}{n h}} \sqrt{B_{K}}+B_{K} h_{f}\right)\right)
\end{aligned}
$$

where the last inequality follows by first combining Lemmas 14, 15, 16 and 17 and plugging in our condition for $r_{b}, h$ and $h_{f}$.

Lemma 14. Under the conditions of Lemma 13, we have

$$
\begin{aligned}
& \max _{l \in[p]} \sup _{\substack{\left\|\delta_{b}\right\|_{0,2} \leq m \\
\left\|\delta_{b}\right\|_{1,2} \leq r_{b}}}\left\|\left(\mathbb{S}_{n}-\mathbb{E} \mathbb{S}_{n}\right)\left[w_{i} \cdot\left(\hat{f}_{i}\left(\delta_{b}\right)-\hat{f}_{i}(0)\right) \cdot A_{i l}\right]\right\|_{F} \\
& \lesssim \frac{B_{K} B_{A}}{h_{f}} \sqrt{\bar{f} B_{X} \frac{r_{b}(m \log p+\log (1 / \gamma))}{n h}}
\end{aligned}
$$

with probability $1-\gamma$, where $A_{i l}$ and $B_{A}$ are defined in Lemma 13.

Proof. Let $\mathcal{W}=\left\{\tilde{W}_{1}, \ldots, \tilde{W}_{K}\right\}$ be the $\frac{1}{2}$-net for $\left\{W \in \mathbb{R}^{2 k \times 2 k} \mid\|W\|_{F} \leq 1\right\}$.

We have that $K \leq 5^{4 k^{2}}$ and

$$
\begin{aligned}
& \max _{l \in[p]} \sup _{\substack{\left\|\delta_{b}\right\|_{0,2} \leq m \\
\left\|\delta_{b}\right\|_{1,2} \leq r_{b}}}\left\|\left(\mathbb{S}_{n}-\mathbb{E S}_{n}\right)\left[w_{i} \cdot\left(\hat{f}_{i}\left(\delta_{b}\right)-\hat{f}_{i}(0)\right) \cdot A_{i l}\right]\right\|_{F} \\
& \leq 2 \cdot \max _{\tilde{W} \in \mathcal{W}} \max _{l \in[p]} \sup _{\substack{\left\|\delta_{b}\right\|_{0,2} \leq m \\
\left\|\delta_{b}\right\|_{1,2} \leq r_{b}}}\left(\mathbb{S}_{n}-\mathbb{E}_{n}\right)\left[w_{i} \cdot\left(\hat{f}_{i}\left(\delta_{b}\right)-\hat{f}_{i}(0)\right) \cdot \operatorname{trace}\left(\tilde{W}^{\top} A_{i l}\right)\right] .
\end{aligned}
$$

Our goal is to apply Lemma 25 to bound the right hand side.

Note that

$$
2 h_{f}\left(\hat{f}_{i}\left(\delta_{b}\right)-\hat{f}_{i}(0)\right)=\mathbb{1}\left\{h_{f}<y_{i}-\Gamma_{i}^{\top} b^{\star} \leq h_{f}+\Gamma_{i}^{\top} \delta_{b}\right\}
$$




$$
\begin{aligned}
& -\mathbb{1}\left\{h_{f}+\Gamma_{i}^{\top} \delta_{b}<y_{i}-\Gamma_{i}^{\top} b^{\star} \leq h_{f}\right\} \\
& -\mathbb{1}\left\{-h_{f} \leq y_{i}-\Gamma_{i}^{\top} b^{\star}<-h_{f}+\Gamma_{i}^{\top} \delta_{b}\right\} \\
& +\mathbb{1}\left\{-h_{f}+\Gamma_{i}^{\top} \delta_{b} \leq y_{i}-\Gamma_{i}^{\top} b^{\star}<-h_{f}\right\} .
\end{aligned}
$$

We proceed to bound

$$
\begin{aligned}
& \max _{\substack{\tilde{W} \in \mathcal{W} \\
l \in[p] \\
|S| \leq m}} \sup _{\substack{\text { suport }\left(\delta_{b}\right)=S \\
\left\|\delta_{b}\right\|_{1,2} \leq r_{b}}} \\
& \quad\left(\mathbb{S}_{n}-\mathbb{E S}_{n}\right)\left[\frac{w_{i}}{2 h_{f}} \cdot \mathbb{1}\left\{h_{f}<y_{i}-\Gamma_{i}^{\top} b^{\star} \leq h_{f}+\Gamma_{i}^{\top} \delta_{b}\right\} \cdot\left|\operatorname{trace}\left(\tilde{W}^{\top} A_{i l}\right)\right|\right],
\end{aligned}
$$

while the other terms are bounded similarly. For a fixed $\tilde{W} \in \mathcal{W}, l \in[p]$ and $|S| \leq m$, define

$$
\begin{aligned}
a_{i} & =\frac{w_{i}}{2 h_{f}} \cdot \operatorname{trace}\left(\tilde{W}^{\top} A_{i l}\right), \text { and } \\
\mathcal{G}_{S}=\left\{\left(y_{i}, x_{i}, u_{i}\right) \mapsto a_{i} \cdot \mathbb{1}\left\{h_{f}<y_{i}-\Gamma_{i}^{\top} b^{\star} \leq h_{f}+\Gamma_{i}^{\top} \delta_{b}\right\}:\right. & \left.\operatorname{support}\left(\delta_{b}\right)=S,\left\|\delta_{b}\right\|_{1,2} \leq r_{b}\right\}, \\
\mathcal{G} & =\cup_{S:|S| \leq m} \mathcal{G}_{S}
\end{aligned}
$$

Let $G(\cdot)$ be an envelope of $\mathcal{G}$ and note that $\|G\|_{\infty} \leq \frac{B_{K} B_{A}}{n h h_{f}}$. For a fixed $g \in \mathcal{G}$, let

$$
g_{i}=g\left(y_{i}, x_{i}, u_{i}\right)=a_{i} \cdot \mathbb{1}\left\{h_{f}<y_{i}-\Gamma_{i}^{\top} b^{\star} \leq h_{f}+\Gamma_{i}^{\top} \delta_{b}\right\} .
$$

We have that

$$
\begin{aligned}
& \mathbb{E}\left[\mathbb{1}\left\{h_{f}<y_{i}-\Gamma_{i}^{\top} b^{\star} \leq h_{f}+\Gamma_{i}^{\top} \delta_{b}\right\}\right] \\
& =F_{i}\left(\Gamma_{i}^{\top} b^{\star}+h_{f}+\Gamma_{i}^{\top} \delta_{b}\right)-F_{i}\left(\Gamma_{i}^{\top} b^{\star}+h_{f}\right) \\
& \leq \bar{f} \cdot\left|\Gamma_{i}^{\top} \delta_{b}\right| \\
& \leq \bar{f} B_{X} r_{b},
\end{aligned}
$$

and, therefore, the variance is bounded as

$$
\sigma_{\mathcal{G}}^{2} \leq \sup _{g \in \mathcal{G}} \sum_{i \in[n]} \mathbb{E}\left[g_{i}^{2}\right] \leq \bar{f} B_{X} r_{b} \sum_{i \in[n]} a_{i}^{2} \leq \bar{f} B_{X} B_{K}^{2} B_{A}^{2} \cdot \frac{r_{b}}{n h h_{f}^{2}}
$$

since

$$
\sum_{i \in[n]} a_{i}^{2} \leq \frac{B_{A}^{2}}{h_{f}^{2}} \sum_{i \in[n]} w_{i}^{2} \leq \frac{B_{K}^{2} B_{A}^{2}}{n h h_{f}^{2}} .
$$

The VC dimension for the space

$$
\mathcal{F}_{S}=
$$




$$
\left\{\left(y_{i}, x_{i}, u_{i}\right) \mapsto \cdot \mathbb{1}\left\{h_{f}<y_{i}-\Gamma_{i}^{\top} b^{\star} \leq h_{f}+\Gamma_{i}^{\top} \delta_{b}\right\}: \operatorname{support}\left(\delta_{b}\right)=S,\left\|\delta_{b}\right\|_{1,2} \leq r_{b}\right\}
$$

is $|S| \leq m$. Therefore, applying Lemma 22 and Lemma 24,

$$
\sup _{Q} \log N\left(\epsilon \cdot \frac{B_{K} B_{A}}{n h h_{f}}, \mathcal{G}_{S},\|\cdot\|_{L_{2}(Q)}\right) \lesssim m \log (1 / \epsilon) .
$$

Since there are $\left(\begin{array}{c}p \\ m\end{array}\right)$ different supports $S$ in $\mathcal{G}$, we have

$$
\sup _{Q} \log N\left(\epsilon \cdot \frac{B_{K} B_{A}}{n h h_{f}}, \mathcal{G},\|\cdot\|_{L_{2}(Q)}\right) \lesssim m(\log (1 / \epsilon)+\log (p)) .
$$

Applying Lemma 21 with $\sigma_{\mathcal{G}}=\frac{B_{K} B_{A}}{h_{f}} \sqrt{\frac{\bar{f} r_{b} B_{X}}{n h}},\|G\|_{\infty} \leq \frac{B_{K} B_{A}}{n h h_{f}}, V=\mathrm{cm}$, and $A=$ $C p^{1 / c}$, we have

$$
\begin{aligned}
& \mathbb{E}\left[\sup _{g \in \mathcal{G}} \sum_{i \in[n]} g_{i}-\mathbb{E}\left[g_{i}\right]\right] \\
& \lesssim\left(m \frac{B_{K} B_{A}}{n h h_{f}} \log \frac{p}{\sqrt{\bar{f} r_{b} B_{X} h}}+\frac{B_{K} B_{A}}{h_{f}} \sqrt{\frac{\bar{f} r_{b} B_{X}}{n h}} \sqrt{m \log \frac{p}{\sqrt{\bar{f} r_{b} B_{X} h}}}\right) \\
& \lesssim \frac{B_{K} B_{A}}{h_{f}} \sqrt{\bar{f} B_{X} \frac{m r_{b} \log p}{n h}}
\end{aligned}
$$

where the last inequality follows from the conditions on $r_{b}$ in Lemma 13 and Assumption 7. Finally, Lemma 25 gives us

$$
\sup _{g \in \mathcal{G}} \sum_{i \in[n]} g_{i}-\mathbb{E}\left[g_{i}\right] \lesssim \frac{B_{K} B_{A}}{h_{f}} \sqrt{\bar{f} B_{X} \frac{r_{b}(m \log p+\log (1 / \gamma))}{n h}},
$$

with probability $1-\gamma$, and, by the union bound over $\tilde{W} \in \mathcal{W}, l \in[p]$,

$$
\begin{gathered}
\max _{\substack{\tilde{W} \in \mathcal{W} \\
l \in[\in] \\
|S| \leq m}} \sup _{\substack{\left.\left\|\delta_{b}\right\|_{1,2} \leq r_{b} \\
|S| \delta_{b}\right)=S}}\left(\mathbb{S}_{n}-\mathbb{E S}_{n}\right)\left[w_{i} \cdot \mathbb{1}\left\{h_{f}<y_{i}-\Gamma_{i}^{\top} b^{\star} \leq h_{f}+\Gamma_{i}^{\top} \delta_{b}\right\} \cdot \operatorname{trace}\left(\tilde{W}^{\top} A_{i l}\right)\right] \\
\lesssim \frac{B_{K} B_{A}}{h_{f}} \sqrt{\bar{f} B_{X} \frac{r_{b}\left(2 m \log p+\log \left(5^{4 k^{2}} / \gamma\right)\right)}{n h}} .
\end{gathered}
$$

Handling other terms in the same way, we obtain

$$
\begin{aligned}
\max _{l \in[p]} \sup _{\substack{\left\|\delta_{b}\right\|_{0,2} \leq m \\
\left\|\delta_{b}\right\|_{1,2} \leq r_{b}}} \|\left(\mathbb{S}_{n}-\mathbb{E} \mathbb{S}_{n}\right)\left[w _ { i } \cdot \left(\hat{f}_{i}\left(\delta_{b}\right)\right.\right. & \left.\left.-\hat{f}_{i}(0)\right) \cdot A_{i l}\right] \|_{F} \\
& \lesssim \frac{B_{K} B_{A}}{h_{f}} \sqrt{\bar{f} B_{X} \frac{r_{b}(m \log p+\log (1 / \gamma))}{n h}},
\end{aligned}
$$

with probability $1-\gamma$, which completes the proof. 
Lemma 15. Under the conditions of Lemma 13, we have

$$
\max _{l \in[p]}\left\|\left(\mathbb{S}_{n}-\mathbb{E S}_{n}\right)\left[w_{i} \cdot \hat{f}_{i}(0) \cdot A_{i l}\right]\right\|_{F} \leq \sqrt{\bar{f} B_{K}^{2} B_{A}^{2} \frac{\log (p / \gamma)}{n h h_{f}}}
$$

with probability $1-\gamma$.

Proof. Let $\mathcal{W}$ be as in the proof of Lemma 14. Then

$$
\begin{aligned}
\max _{l \in[p]}\left\|\left(\mathbb{S}_{n}-\mathbb{E S}_{n}\right)\left[w_{i} \cdot \hat{f}_{i}(0) \cdot A_{i l}\right]\right\|_{F} \\
\quad \leq 2 \max _{\tilde{W} \in \mathcal{W}} \max _{l}\left(\mathbb{S}_{n}-\mathbb{E S}_{n}\right)\left[w_{i} \cdot \hat{f}_{i}(0) \cdot \operatorname{trace}\left(\tilde{W}^{\top} A_{i l}\right)\right] .
\end{aligned}
$$

Let $Z_{i}=w_{i} \cdot \hat{f}_{i}(0) \cdot \operatorname{trace}\left(\tilde{W}^{\top} A_{i l}\right)$. Then

$$
\begin{aligned}
\sum_{i \in[n]} \mathbb{E}\left[Z_{i}^{2}\right] \leq \sum_{i \in[n]} \frac{w_{i}^{2}}{h_{f}^{2}} \cdot \operatorname{trace}\left(\tilde{W}^{\top} A_{i l}\right)^{2} \cdot \mathbb{E}\left[\mathbb { 1 } \left\{\left|y_{i}-\Gamma_{i}^{\top} b^{\star}\right|\right.\right. & \left.\left.\leq h_{f}\right\}\right] \\
& =O\left(\frac{\bar{f} B_{K}^{2} B_{A}^{2}}{n h h_{f}}\right),
\end{aligned}
$$

and

$$
\max _{i \in[n]}\left|Z_{i}\right| \leq O\left(\frac{\bar{f} B_{K} B_{A}}{n h h_{f}}\right) .
$$

The result follows from Lemma 25 and the union bound.

Lemma 16. Suppose conditions of Lemma 13 hold. Then

$$
\max _{l \in[p]}\left\|E_{l}^{\top}\left(\mathbb{E}\left[\widehat{H}\left(\delta_{b}\right)\right]-\mathbb{E}[\widehat{H}(0)]\right) V^{\star}\right\|_{F} \leq \bar{f}^{\prime} B_{A}\left(s \sqrt{\frac{B_{K} \log (n p)}{n h}}+B_{K} h_{f}\right) .
$$

Proof. For a fixed $\delta_{b}$, the mean value theorem gives us

$$
\begin{aligned}
& 2 h_{f}\left|\mathbb{E}\left[\hat{f}_{i}\left(\delta_{b}\right)\right]-\mathbb{E}\left[\hat{f}_{i}(0)\right]\right| \\
= & \mid F_{i}\left(\Gamma_{i}^{\top}\left(b^{\star}+\delta_{b}\right)+h_{f}\right)-F_{i}\left(\Gamma_{i}^{\top}\left(b^{\star}+\delta_{b}\right)-h_{f}\right) \\
& -F_{i}\left(\Gamma_{i}^{\top} b^{\star}+h_{f}\right)+F_{i}\left(\Gamma_{i}^{\top} b^{\star}-h_{f}\right) \mid \\
\leq & 2 h_{f} \bar{f}^{\prime}\left(\left|\Gamma_{i}^{\top} \delta_{b}\right|+h_{f}\right),
\end{aligned}
$$

Therefore, we have that

$$
\begin{gathered}
\max _{l \in[p]}\left\|E_{l}^{\top}\left(\mathbb{E}\left[\widehat{H}\left(\delta_{b}\right)\right]-\mathbb{E}[\widehat{H}(0)]\right) V^{\star}\right\|_{F} \\
\leq \bar{f}^{\prime} B_{A} \sum_{i \in[n]} w_{i} \cdot\left(\left|\Gamma_{i}^{\top} \delta_{b}\right|+h_{f}\right)
\end{gathered}
$$




$$
\begin{aligned}
& \leq \bar{f}^{\prime} B_{A}\left(\sqrt{\sum_{i \in[n]} w_{i}\left(\Gamma_{i}^{\top} \delta_{b}\right)^{2}} \sqrt{\sum_{i \in[n]} w_{i}}+h_{f} \sum_{i \in[n]} w_{i}\right) \\
& \leq \bar{f}^{\prime} B_{A}\left(s \sqrt{\frac{\log (n p)}{n h}} \sqrt{B_{K}}+B_{K} h_{f}\right),
\end{aligned}
$$

where the last inequality follows from Lemma 12 and Assumption 6.

Lemma 17. Suppose conditions of Lemma 13 hold. Then

$$
\max _{l \in[p]}\left\|E_{l}^{\top}\left(\mathbb{E}[\widehat{H}(0)]-H^{\star}\right) V^{\star}\right\|_{F} \leq \bar{f}^{\prime} B_{A}\left(B_{K} h_{f}+2\left(h^{4} s B_{X} B_{\beta}+\frac{\epsilon_{R}^{2}}{\underline{f}^{2}}\right)^{1 / 2}\right) .
$$

Proof. The mean value theorem gives us

$$
\begin{aligned}
2 h_{f} \mathbb{E}\left[\hat{f}_{i}(0)\right] & =F_{i}\left(\Gamma_{i}^{\top} b^{\star}+h_{f}\right)-F_{i}\left(\Gamma_{i}^{\top} b^{\star}-h_{f}\right) \\
& =2 h_{f} \cdot f_{i}\left(q_{i}\right)+2 h_{f} \cdot\left(f_{i}\left(\check{q}_{i}\right)-f_{i}\left(q_{i}\right)\right),
\end{aligned}
$$

where $\check{q}_{i}$ is a point between $\Gamma_{i}^{\top} b^{\star}-h_{f}$ and $\Gamma_{i}^{\top} b^{\star}+h_{f}$. Therefore, we have

$$
\begin{gathered}
\left|\mathbb{E}\left[\hat{f}_{i}(0)\right]-f_{i}\left(q_{i}\right)\right| \leq \bar{f}^{\prime}\left|\check{q}_{i}-q_{i}\right| \leq \bar{f}^{\prime}\left(\left|\tilde{q}_{i}-q_{i}\right|+h_{f}\right), \text { and } \\
\left|f_{i}\left(\tilde{q}_{i}\right)-f_{i}\left(q_{i}\right)\right| \leq \bar{f}^{\prime}\left|\tilde{q}_{i}-q_{i}\right| .
\end{gathered}
$$

Finally, we have

$$
\begin{aligned}
\max _{l \in[p]} & \left\|E_{l}^{\top}\left(\mathbb{E}[\widehat{H}(0)]-H^{\star}\right) V^{\star}\right\|_{F} \\
& \leq \bar{f}^{\prime} B_{A} \sum_{i \in[n]} w_{i} \cdot\left(2\left|\tilde{q}_{i}-q_{i}\right|+h_{f}\right) \\
& \leq \bar{f}^{\prime} B_{A}\left(h_{f} \sum_{i \in[n]} w_{i}+2\left(\sum_{i \in[n]} w_{i}\right)^{1 / 2} \cdot\left(\sum_{i \in[n]} w_{i}\left(\tilde{q}_{i}-q_{i}\right)^{2}\right)^{1 / 2}\right) \\
& \leq \bar{f}^{\prime} B_{A} B_{K}\left(h_{f}+2\left(h^{4} s B_{X}^{2} B_{\beta}+\frac{\epsilon_{R}^{2}}{\frac{f^{2}}{2}}\right)^{1 / 2}\right),
\end{aligned}
$$

where the last inequality follows from Lemma 12 and Assumption 6.

Lemma 18. Assume $B_{V}$ satisfies Assumption 5, that $(n h)^{-1} s B_{V}^{2} \log p=o(1)$ and $\log \left(B_{V}^{2} h_{f} h\right)=O(\log p)$. Let $S_{1}$ be the support of $b^{\star}$ and $S_{2}$ be the support of $V^{\star}$ as defined in Assumption 6, with $\left|S_{1}\right|=s_{1}$ and $\left|S_{2}\right|=s_{2}$. Define

$$
\begin{aligned}
\Delta_{b}\left(r_{b}, s_{1}\right) & =\left\{\delta \in \mathbb{R}^{2 p},\|\delta\|_{2} \leq r_{b},\|\delta\|_{0} \leq s_{1}\right\} \quad \text { and } \\
\mathbb{C}(S) & =\left\{\Theta \in \mathbb{R}^{2 k \times 2 p}:\left\|\Theta_{S^{c}}\right\|_{1, F} \leq 6\left\|\Theta_{S}\right\|_{1, F}\right\} .
\end{aligned}
$$


Then

$$
\operatorname{trace}\left(\delta_{v}^{\top} \widehat{H}\left(\delta_{b}\right) \delta_{v}\right) \geq \underline{f} \kappa_{-}\left\|\delta_{v}\right\|_{F}^{2}-o_{p}(1)\left(\left\|\delta_{v}\right\|_{F}+\frac{\left\|\delta_{v}\right\|_{1, F}}{\sqrt{s_{2}}}\right)^{2}
$$

for all $\delta_{b} \in \Delta_{b}\left(r_{b}, s_{1}\right)$ and $\delta_{v} \in \mathbb{C}\left(S_{2}\right)$.

Proof. For a fixed $\delta_{b}$, we have that $f_{i}\left(\delta_{b}\right) \geq \underline{f}$ and $\delta_{v} \in \mathbb{C}\left(S_{2}\right)$. Therefore,

$$
\operatorname{trace}\left(\delta_{v}^{\top} H\left(\delta_{b}\right) \delta_{v}\right) \geq \underline{f} \sum_{i \in[n]} w_{i} \operatorname{trace}\left(\delta_{v}^{\top} \Gamma_{i} \Gamma_{i}^{\top} \delta_{v}\right) \geq \underline{f} \kappa_{-}^{2}\left\|\delta_{v}\right\|_{F}^{2}
$$

The proof now follows from Lemma 19 and Lemma 20 (presented next),

$$
\begin{aligned}
& \operatorname{trace}\left(\delta_{v}^{\top} \widehat{H}\left(\delta_{b}\right) \delta_{v}\right) \\
& \geq \operatorname{trace}\left(\delta_{v}^{\top} H\left(\delta_{b}\right) \delta_{v}\right)-\operatorname{trace}\left(\delta_{v}^{\top}\left(\widehat{H}\left(\delta_{b}\right)-H\left(\delta_{b}\right)\right) \delta_{v}\right) \\
& \geq \operatorname{trace}\left(\delta_{v}^{\top} H\left(\delta_{b}\right) \delta_{v}\right) \\
& \quad-\sup _{\delta \in \Delta_{v}\left(s_{2}\right)} \sup _{b} \in \Delta_{b}\left(r_{b}, s_{1}\right) \\
& \geq \underline{f} \kappa_{-}^{2}\left\|\delta_{v}\right\|_{F}^{2}-o_{p}(1)\left(\left\|\delta_{v}\right\|_{F}+\frac{\left\|\delta_{v}\right\|_{1, F}}{\sqrt{s_{2}}}\right)^{2},
\end{aligned}
$$

where $\Delta_{v}\left(s_{2}\right)$ is as defined in Lemma 20 .

Lemma 19 (Based on proposition 5 in (Sun and Zhang, 2013)). For any fixed matrix $M \in R^{p \times p}$ and matrices $u \in \mathbb{R}^{k \times p}$ and $s \in \mathbb{N}$,

$$
\operatorname{trace}\left(u^{\top} M u\right) \leq\left(\|u\|_{F}+\frac{\|u\|_{1, F}}{\sqrt{s}}\right)^{2}\|M\|_{\mathcal{S}_{s}},
$$

where $\mathcal{S}_{s}=\left\{u \in \mathbb{R}^{k \times p} \mid\|u\|_{F}=1,\|u\|_{0, F} \leq s\right\}$ and

$$
\|M\|_{\mathcal{S}_{s}}=\max _{u, v \in \mathcal{S}_{s}} \operatorname{trace}\left(u^{\top} M v\right)
$$

Lemma 20. Under the conditions of Lemma 18, we have

$$
\begin{array}{r}
\sup _{\delta_{v} \in \Delta_{v}\left(s_{2}\right)} \sup _{\delta_{b} \in \Delta_{b}\left(r_{b}, s_{1}\right)}\left|\operatorname{trace}\left(\delta_{v}^{\top}\left(\widehat{H}\left(\delta_{b}\right)-H\left(\delta_{b}\right)\right) \delta_{v}\right)\right| \\
=O_{p}\left(\sqrt{\frac{\left(s_{1}+s_{2}\right) \bar{f} \kappa_{+} B_{K} B_{V}^{2} \log (p)}{n h h_{f}}}\right),
\end{array}
$$

where

$$
\begin{gathered}
\Delta_{v}\left(s_{2}\right)=\left\{\delta \in \mathbb{R}^{2 k \times 2 p},\|\delta\|_{F}=1,\|\delta\|_{0, F} \leq s_{2}\right\} \\
\Delta_{b}\left(r_{b}, s_{1}\right)=\left\{\delta \in \mathbb{R}^{2 p},\|\delta\|_{2} \leq r_{b},\|\delta\|_{0} \leq s_{1}\right\} .
\end{gathered}
$$


Proof. We have

$$
\begin{aligned}
& \sup _{\delta_{v} \in \Delta_{v}\left(s_{2}\right)} \sup _{\delta_{b} \in \Delta_{b}\left(r_{b}, s_{1}\right)}\left|\operatorname{trace}\left(\delta_{v}^{\top}\left(\widehat{H}\left(\delta_{b}\right)-H\left(\delta_{b}\right)\right) \delta_{v}\right)\right| \\
= & \sup _{\delta_{v} \in \Delta_{v}\left(s_{2}\right)} \sup _{\delta_{b} \in \Delta_{b}\left(r_{b}, s_{1}\right)} \\
& \left(\mathbb{S}_{n}-\mathbb{E S}_{n}\right)\left\{w_{i}\left(2 h_{f}\right)^{-1} \cdot \mathbb{1}\left\{\left|y_{i}-\Gamma_{i}^{\top}\left(b^{\star}+\delta_{b}\right)\right| \leq h_{f}\right\}\right\} \cdot\left\|\delta_{v} \Gamma_{i}\right\|_{2}^{2} \\
\leq & 2 \sup _{\delta_{v} \in \mathcal{N}_{\epsilon}} \sup _{\delta_{b} \in \Delta_{b}\left(r_{b}, s_{1}\right)} \\
& \left(\mathbb{S}_{n}-\mathbb{E S}_{n}\right)\left\{w_{i}\left(2 h_{f}\right)^{-1} \cdot \mathbb{1}\left\{\left|y_{i}-\Gamma_{i}^{\top}\left(b^{\star}+\delta_{b}\right)\right| \leq h_{f}\right\}\right\} \cdot\left\|\delta_{v} \Gamma_{i}\right\|_{2}^{2},
\end{aligned}
$$

where $\mathcal{N}_{\epsilon}$ is an $\epsilon$-net for $\Delta_{v}\left(s_{2}\right)$. We have $\left|\mathcal{N}_{\epsilon}\right| \leq 5^{2 k s_{2}}$. Fix $\delta_{v} \in \mathcal{N}_{\epsilon}$. Define

$$
\begin{aligned}
a_{i}= & \left(2 h_{f}\right)^{-1} w_{i} \cdot \operatorname{trace}\left\{\delta_{v} \Gamma_{i} \Gamma_{i}^{\top} \delta_{v}^{\top}\right\}, \\
\mathcal{G}_{S}= & \left\{\left(y_{i}, x_{i}, u_{i}\right) \mapsto a_{i} \cdot \mathbb{1}\left\{\left|y_{i}-\Gamma_{i}^{\top}\left(b^{\star}+\delta_{b}\right)\right| \leq h_{f}\right\}:\right. \\
& \left.\operatorname{support}\left(\delta_{b}\right)=S,\left\|\delta_{b}\right\|_{2} \leq r_{b}\right\}, \\
\mathcal{G}= & \cup_{S:|S| \leq s_{1}} \mathcal{G}_{S} .
\end{aligned}
$$

Let $G(\cdot)=\frac{B_{K} B_{V}^{2}}{2 n h h_{f}}$ be an envelope of $\mathcal{G}$. For a fixed $g \in \mathcal{G}$, let

$$
g_{i}=g\left(y_{i}, x_{i}, u_{i}\right)=a_{i} \cdot \mathbb{1}\left\{\left|y_{i}-\Gamma_{i}^{\top}\left(b^{\star}+\delta_{b}\right)\right| \leq h_{f}\right\} .
$$

Therefore, the variance is bounded as

$$
\sigma_{\mathcal{G}}^{2} \leq \sup _{g \in \mathcal{G}} \sum_{i \in[n]} \mathbb{E}\left[g_{i}^{2}\right] \lesssim \bar{f} h_{f} \sum_{i \in[n]}\left(4 h_{f}^{2}\right)^{-1} w_{i}^{2} \cdot\left\|\delta_{v} \Gamma_{i}\right\|_{2}^{4} \lesssim \frac{\bar{f} \kappa_{+} B_{K} B_{V}^{2}}{n h h_{f}}
$$

The VC dimension for the space $\mathcal{G}_{S}$ is $O(|S|)$. Therefore, using Lemma 22,

$$
\sup _{Q} \log N\left(\epsilon, \mathcal{G},\|\cdot\|_{L_{2}(Q)}\right) \lesssim s_{1}(\log (p)+\log (1 / \epsilon)) .
$$

Applying Lemma 21 we obtain

$$
\mathbb{E}\left[\sup _{g \in \mathcal{G}} \sum_{i \in[n]} g_{i}-\mathbb{E}\left[g_{i}\right]\right] \lesssim \sqrt{\frac{s_{1} \bar{f} \kappa_{+} B_{K} B_{V}^{2} \log (p)}{n h h_{f}}},
$$

under our assumptions. Using Lemma 25,

$$
\sup _{g \in \mathcal{G}} \sum_{i \in[n]} g_{i}-\mathbb{E}\left[g_{i}\right]=O_{p}\left(\sqrt{\frac{s_{1} B_{V}^{2} \log (p)}{n h h_{f}}}\right) .
$$

A union bound over $\mathcal{N}_{\epsilon}$ completes the proof. 


\section{A.8. Empirical Process Results}

Definition 1. The covering number $N(\epsilon, \mathcal{F},\|\cdot\|)$ is the minimal number of balls $\{g \mid\|g-f\| \leq \epsilon\}$ of radius $\epsilon$ needed to cover the set $\mathcal{F}$.

Let $\|\mathcal{F}\|_{\infty}=\sup \left\{\|f\|_{\infty}, f \in \mathcal{F}\right\}$. Furthermore, define

$$
\Sigma_{\mathcal{F}}^{2}=\mathbb{E}\left[\sup _{f \in \mathcal{F}} \sum_{i \in[n]} W_{i}^{2}(f)\right] \quad \text { and } \quad \sigma_{\mathcal{F}}^{2}=\sup _{f \in \mathcal{F}} \sum_{i \in[n]} \mathbb{E}\left[W_{i}^{2}(f)\right]
$$

where $W_{i}(f), f \in \mathcal{F}, i \in[n]$ are real valued random variables.

Lemma 21. Let $\mathcal{F}$ be a measurable uniformly bounded class of functions satisfying

$$
N\left(\epsilon\|F\|_{L_{2}(P)}, \mathcal{F}, L_{2}(P)\right) \leq\left(\frac{A}{\epsilon}\right)^{V}
$$

for all probability measures $P$, where $F:=\sup _{f \in \mathcal{F}}|f|$ is the envelope function and $A, V$ are constants dependent on $\mathcal{F}$. Let $\sigma_{\mathcal{F}}^{2}=\sup _{f \in \mathcal{F}} \sum_{i \in n} \mathbb{E}\left[\left(f_{i}-\mathbb{E}\left[f_{i}\right]\right)^{2}\right]$ and $U \geq \sup _{f \in \mathcal{F}}\|f\|_{\infty}$ be such that $0<\sigma_{\mathcal{F}} \leq \sqrt{n} U$. Then there exists a universal constant $C$ such that

$$
\mathbb{E}\left[\sup _{f \in \mathcal{F}} \sum_{i \in[n]} f_{i}-\mathbb{E}\left[f_{i}\right]\right] \leq C\left[V U \log \frac{\sqrt{n} A U}{\sigma_{\mathcal{F}}}+\sigma_{\mathcal{F}} \sqrt{V \log \frac{\sqrt{n} A U}{\sigma_{\mathcal{F}}}}\right] .
$$

Proof. This is essentially Proposition 2.1 of Giné and Guillou (2001) combined with symmetrization Koltchinskii and Yuan (2010).

Lemma 22 (Theorem 2.6.7 of van der Vaart and Wellner (1996)). Suppose $\mathcal{F}$ is a function class with a bounded $V C$-dimension, $V$, and an envelope $F$. Then there exist absolute constants $c, C>0$ such that

$$
\sup _{Q} N\left(\epsilon\|F\|_{Q, 2}, \mathcal{F},\|\cdot\|_{L_{2}(Q)}\right) \leq\left(\frac{C}{\epsilon}\right)^{c V}
$$

for all $\epsilon \in(0,1)$ and the probability measure $Q$ ranges over distributions such that $\|F\|_{Q, 2}>0$.

Lemma 23 (Lemma 22 of Nolan and Pollard (1987)). Let $K: \mathbb{R} \mapsto \mathbb{R}$ be a bounded variation function. The function class

$$
\mathcal{K}=\left\{K\left(\frac{s-\cdot}{h}\right) \mid h>0, s \in \mathbb{R}\right\},
$$

indexed by the kernel bandwidth and the location s, satisfies the uniform entropy condition

$$
\sup _{Q} N\left(\epsilon, \mathcal{K},\|\cdot\|_{L_{2}(Q)}\right) \leq C \epsilon^{-v}, \quad \text { for all } \epsilon \in(0,1),
$$

for some $C>0$ and $v>0$. 
Lemma 24 (Lemma 26 of Lu et al. (2018)). Let $\mathcal{F}$ and $\mathcal{G}$ be two function classes satisfying

$$
\sup _{Q} N\left(a_{1} \epsilon, \mathcal{F},\|\cdot\|_{L_{2}(Q)}\right) \leq C_{1} \epsilon^{-v_{1}} \quad \text { and } \quad \sup _{Q} N\left(a_{2} \epsilon, \mathcal{G},\|\cdot\|_{L_{2}(Q)}\right) \leq C_{2} \epsilon^{-v_{2}}
$$

for some $C_{1}, C_{2}, a_{1}, a_{2}, v_{1}, v_{2}>0$ and any $0<\epsilon<1$. Define

$$
\mathcal{F}_{\times}=\{f g \mid f \in \mathcal{F}, g \in \mathcal{G}\} \quad \text { and } \quad \mathcal{F}_{+}=\{f+g \mid f \in \mathcal{F}, g \in \mathcal{G}\} .
$$

Then for any $\epsilon \in(0,1)$,

$$
\sup _{Q} N\left(\epsilon, \mathcal{F}_{\times},\|\cdot\|_{L_{2}(Q)}\right) \leq C_{1} C_{2}\left(\frac{2 a_{1} U}{\epsilon}\right)^{v_{1}}\left(\frac{2 a_{2} U}{\epsilon}\right)^{v_{2}}
$$

and

$$
\sup _{Q} N\left(\epsilon, \mathcal{F}_{+},\|\cdot\|_{L_{2}(Q)}\right) \leq C_{1} C_{2}\left(\frac{2 a_{1}}{\epsilon}\right)^{v_{1}}\left(\frac{2 a_{2}}{\epsilon}\right)^{v_{2}}
$$

where $U=\|\mathcal{F}\|_{\infty} \vee\|\mathcal{G}\|_{\infty}$.

Lemma 25. Let

$$
Z=\sup _{f \in \mathcal{F}} \sum_{i \in[n]} W_{i}(f)
$$

where $\mathbb{E}\left[W_{i}(f)\right]=0$ and $\left|W_{i}(f)\right| \leq M$ for all $i \in[n]$ and $f \in \mathcal{F}$. Then

$$
Z \leq \mathbb{E}[Z]+4\left(\sqrt{\left(4 M \mathbb{E}[Z]+\sigma_{\mathcal{F}}^{2}\right) \log (1 / \delta)} \bigvee M \log (1 / \delta)\right)
$$

with probability $1-\delta$.

Proof. The lemma is a simple consequence of Theorems 11.8 and 12.2 in Boucheron et al. (2013). Assume that $M=1$. Then Theorem 12.2 in Boucheron et al. (2013) gives us

$$
\mathbb{P}\{Z \geq \mathbb{E}[Z]+t\} \leq \exp \left(-\frac{t^{2}}{2\left(2\left(\Sigma_{\mathcal{F}}^{2}+\sigma_{\mathcal{F}}^{2}\right)+t\right)}\right) .
$$

Hence, with probability $1-\delta$, we have

$$
Z \leq \mathbb{E}[Z]+\sqrt{8\left(\Sigma_{\mathcal{F}}^{2}+\sigma_{\mathcal{F}}^{2}\right) \log (1 / \delta)} \bigvee 4 \log (1 / \delta) .
$$

Furthermore, Theorem 11.8 in Boucheron et al. (2013) gives us that

$$
\Sigma_{\mathcal{F}}^{2}+\sigma_{\mathcal{F}}^{2} \leq 8 \mathbb{E}[Z]+2 \sigma_{\mathcal{F}}^{2} .
$$

Combining with the display above, we get

$$
Z \leq \mathbb{E}[Z]+4\left(\sqrt{\left(4 \mathbb{E}[Z]+\sigma_{\mathcal{F}}^{2}\right) \log (1 / \delta)} \bigvee \log (1 / \delta)\right)
$$

with probability $1-\delta$. We can rescale the equation above by $M$ to conclude the proof of the lemma. 


\section{Appendix B: Numerical studies}

Detailed data settings In our numerical study, we set the parameters

$$
\left(a_{0}, a_{1}, b_{0}, b_{1}, c_{0}, c_{1}, d_{0}, d_{1}, \rho, \sigma_{e}\right)=(1,0.1,1,0.1,1,0.5,1,0.2,0.2,1)
$$

to represent a general setting. We study the cases where $\gamma=0$ and $\gamma=1$, and choose $c_{y}$ and $c_{x}$ to form different combinations of $\left(R_{y}^{2}, R_{x}^{2}\right)$.

Additional simulation results In Table 2, we present the performance of methods with different combinations of $\left(R_{x}^{2}, R_{y}^{2}\right)$.

TABLE 2

Simulation results with data settings varying $\left(R_{x}^{2}, R_{y}^{2}\right)$ with heterogeneous $\epsilon$ (i.e. $\gamma=1$ ).

\begin{tabular}{|c|c|c|c|c|c|c|}
\hline$\epsilon$ distribution & $\left(R_{x}^{2}, R_{y}^{2}\right)$ & Method & Bias & SD & ESE & $\mathrm{CR}$ \\
\hline \multirow{15}{*}{ Normal } & \multirow{5}{*}{$(0.3,0.3)$} & One Step & -0.007 & 0.081 & 0.080 & 0.94 \\
\hline & & Decorrelated score & 0.003 & 0.077 & 0.092 & 0.98 \\
\hline & & Reparameterization & 0.011 & 0.081 & 0.080 & 0.94 \\
\hline & & Naive & 0.017 & 0.089 & 0.090 & 0.96 \\
\hline & & Oracle & -0.012 & 0.075 & 0.091 & 0.97 \\
\hline & \multirow{5}{*}{$(0.3,0.7)$} & One Step & 0.001 & 0.111 & 0.095 & 0.93 \\
\hline & & Decorrelated score & 0.05 & 0.090 & 0.110 & 0.97 \\
\hline & & Reparameterization & 0.05 & 0.088 & 0.095 & 0.93 \\
\hline & & Naive & 0.260 & 0.215 & 0.095 & 0.47 \\
\hline & & Oracle & -0.012 & 0.076 & 0.091 & 0.97 \\
\hline & \multirow{5}{*}{$(0.7,0.7)$} & One Step & -0.029 & 0.179 & 0.188 & 0.96 \\
\hline & & Decorrelated score & 0.155 & 0.202 & 0.249 & 0.92 \\
\hline & & Reparameterization & 0.068 & 0.162 & 0.188 & 0.95 \\
\hline & & Naive & 0.388 & 0.248 & 0.146 & 0.38 \\
\hline & & Oracle & -0.013 & 0.183 & 0.197 & 0.95 \\
\hline \multirow{15}{*}{$t(3)$} & \multirow{5}{*}{$(0.3,0.3)$} & One Step & 0.008 & 0.132 & 0.139 & 0.96 \\
\hline & & Decorrelated score & 0.010 & 0.130 & 0.145 & 0.96 \\
\hline & & Reparameterization & 0.008 & 0.130 & 0.139 & 0.94 \\
\hline & & Naive & 0.008 & 0.130 & 0.148 & 0.98 \\
\hline & & Oracle & -0.010 & 0.098 & 0.119 & 0.97 \\
\hline & \multirow{5}{*}{$(0.3,0.7)$} & One Step & -0.007 & 0.118 & 0.126 & 0.96 \\
\hline & & Decorrelated score & 0.016 & 0.112 & 0.145 & 0.97 \\
\hline & & Reparameterization & 0.024 & 0.102 & 0.126 & 0.95 \\
\hline & & Naive & 0.066 & 0.163 & 0.125 & 0.90 \\
\hline & & Oracle & -0.010 & 0.100 & 0.119 & 0.97 \\
\hline & \multirow{5}{*}{$(0.7,0.7)$} & One Step & -0.027 & 0.382 & 0.441 & 0.95 \\
\hline & & Decorrelated score & 0.032 & 0.328 & 0.454 & 0.95 \\
\hline & & Reparameterization & 0.031 & 0.302 & 0.441 & 0.97 \\
\hline & & Naive & 0.045 & 0.314 & 0.318 & 0.93 \\
\hline & & Oracle & 0.001 & 0.211 & 0.268 & 0.98 \\
\hline
\end{tabular}

\section{Appendix C: Remarks on Assumption 5}

Assumption 5 holds when $X_{A}$ follows a multivariate approximately sparse linear model, where we require the coefficients to be approximately linear, sparse, 
and smooth. Specifically, we assume that there exists a smooth and sparse $\zeta_{1}^{\star}(u), \cdots, \zeta_{k}^{\star}(u)$, that is,

- $u \mapsto \zeta_{j}^{\star}(u)$ is differentiable for $j=1, \cdots, k$ and

$$
\begin{gathered}
\left\|\zeta_{j}^{\star}\left(u^{\prime}\right)-\zeta_{j}^{\star}(u)\right\|_{2} \leq B_{\zeta}\left\|u^{\prime}-u\right\| ; \\
\left\|\zeta_{j}^{\star}\left(u^{\prime}\right)-\zeta_{j}^{\star}(u)-\left(u^{\prime}-u\right) \cdot \nabla_{u} \zeta_{j}^{\star}(u)\right\|_{2} \leq B_{\zeta}\left(u^{\prime}-u\right)^{2} ;
\end{gathered}
$$

- the supports of $\beta^{\star}(u, \tau)$ and $\partial_{u} \beta^{\star}(\tau, u)$ are sparse, i.e.

$$
\begin{gathered}
S_{j}:=\left\{j \in[p] \mid \zeta_{j}^{\star}(u) \neq 0\right\} \text { and } \\
S_{j}^{\prime}:=\left\{j \in[p] \mid \zeta_{j}^{\star}(u) \neq 0 \text { or } \partial_{u} \zeta_{j}^{\star}(u) \neq 0\right\},
\end{gathered}
$$

$s_{j}:=|S| \ll n$ and $\left|S^{\prime}\right| \leq s_{1 j}:=c_{1 j} s_{j}$ for some constants $c_{1 j}$; such that

with $\zeta^{\star}(u)=\left(\zeta_{1}^{\star}(u), \cdots, \zeta_{k}^{\star}(u)\right)^{\top}$, the residual $r_{i}=x_{i, A}-\zeta^{\star}\left(u_{i}\right) x_{i, A^{c}}$ is approximately orthogonal to $x_{i, A^{c}}$ weighted by $w_{i} f_{i}\left(\tilde{q}_{i}\right)$. Specifically,

$$
\begin{aligned}
& \max \left\{\left\|\sum_{i} w_{i} f_{i}\left(\tilde{q}_{i}\right) r_{i} x_{i, A^{c}}^{\top}\right\|_{\infty},\right. \\
& \left.\left\|\sum_{i} w_{i} f_{i}\left(\tilde{q}_{i}\right) r_{i} \frac{u_{i}-u}{h} x_{i, A^{c}}^{\top}\right\|_{\infty},\left\|\sum_{i} w_{i} f_{i}\left(\tilde{q}_{i}\right) r_{i} \frac{\left(u_{i}-u\right)^{2}}{h^{2}} x_{i, A^{c}}^{\top}\right\|_{\infty}\right\} \\
& =\epsilon_{r}^{2}=O\left(\frac{\log (n p)}{n h h_{f}}\right) .
\end{aligned}
$$

Based on our model assumption, for a fixed $u$, let

$$
r^{\star}=r^{\star}(u)=\left(\begin{array}{cc}
\zeta^{\star}(u) & h \nabla_{u} \zeta^{\star}(u) \\
0 & \zeta^{\star}(u)
\end{array}\right) .
$$

We have the following sparse linear regression model

$$
w_{i} f_{i}\left(\tilde{q}_{i}\right)\left(\begin{array}{c}
x_{i, A} \\
\frac{u_{i}-u}{h} x_{i, A}
\end{array}\right)=w_{i} f_{i}\left(\tilde{q}_{i}\right) r^{\star}\left(\begin{array}{c}
x_{i, A^{c}} \\
\frac{u_{i}-u}{h} x_{i, A^{c}}
\end{array}\right)+w_{i} f_{i}\left(\tilde{q}_{i}\right) \tilde{r}_{i}
$$

where

$$
\tilde{r}_{i}=\left(\begin{array}{c}
r_{i}+\left(\zeta^{\star}\left(u_{i}\right)-\zeta^{\star}(u)-\left(u_{i}-u\right) \cdot \nabla_{u} \zeta^{\star}(u)\right) x_{i, A^{c}} \\
\frac{u-u_{i}}{h}\left(r_{i}+\left(\zeta^{\star}\left(u_{i}\right)-\zeta^{\star}(u)\right) x_{i, A^{c}}\right)
\end{array}\right) .
$$

If $\Sigma_{11}=\sum_{i} w_{i} f_{i}\left(\tilde{q}_{i}\right) \tilde{r}_{i} \tilde{r}_{i}^{\top}$ is invertible, then we can have a specific $V^{\star}$ in the form of $\Sigma_{11}^{-1}\left(I_{2 k},-r^{\star}\right)$ satisfy Assumption 5 . The sparsity of $\zeta^{\star}(u)$ guarantees the sparsity of $V^{\star}$, so we just need to show $\left\|H^{\star} V^{\star}-E_{a}\right\|_{\infty, F} \leq \lambda^{\star}$, where the norm $\|\cdot\|_{\infty, F}$ for $V \in \mathbb{R}^{2 k \times 2 p}$ is defined as $\|V\|_{\infty, F}=\sup _{i \in[k], j \in[p]}\left\|V_{(i, i+k),(j, j+p)}\right\|_{F}$. If we show that

$$
\left\|\Sigma_{11}^{-1} \sum_{i} w_{i} f_{i}\left(\tilde{q}_{i}\right) \tilde{r}_{i} \Gamma_{i, A^{c}}^{\top}\right\|_{\infty, F} \leq \lambda^{\star},
$$

then

$$
\left\|\Sigma_{11}^{-1} \sum_{i} w_{i} f_{i}\left(\tilde{q}_{i}\right) \tilde{r}_{i} \Gamma_{i, A}^{\top}-I_{2 k}\right\|_{\infty, F}
$$




$$
\begin{aligned}
& =\left\|\Sigma_{11}^{-1}\left(\sum_{i} w_{i} f_{i}\left(\tilde{q}_{i}\right) \tilde{r}_{i} \Gamma_{i, A}^{\top}-\sum_{i} w_{i} f_{i}\left(\tilde{q}_{i}\right) \tilde{r}_{i} \tilde{r}_{i}^{\top}\right)\right\|_{\infty, F} \\
& =\left\|\Sigma_{11}^{-1}\left(\sum_{i} w_{i} f_{i}\left(\tilde{q}_{i}\right) \tilde{r}_{i} \Gamma_{i, A^{c}}^{\top}\right)\right\|_{\infty, F} \leq \lambda^{\star}
\end{aligned}
$$

Since $\Sigma_{11}$ is invertible, it is sufficient to bound $\left\|\sum_{i} w_{i} f_{i}\left(\tilde{q}_{i}\right) \tilde{r}_{i} \Gamma_{i, A^{c}}^{\top}\right\|_{\infty, F}$. Given that

$$
\begin{aligned}
& \left\|\sum_{i} w_{i} f_{i}\left(\tilde{q}_{i}\right) \tilde{r}_{i} \Gamma_{i, A^{c}}^{\top}\right\|_{\infty, F} \\
& \leq \sup _{l \in[k], j \in[p-k]} \\
& \left\|\sum_{i} w_{i} f_{i}\left(\tilde{q}_{i}\right)\left(\begin{array}{c}
r_{i l}+x_{i, A^{c}}\left(\zeta_{l}^{\star}\left(u_{i}\right)-\zeta_{l}^{\star}(u)-\left(u_{i}-u\right) \nabla_{u} \zeta_{l}^{\star}(u)\right) \\
\frac{u-u_{i}}{h}\left(r_{i l}+x_{i, A^{c}}\left(\zeta_{l}^{\star}\left(u_{i}\right)-\zeta_{l}^{\star}(u)\right)\right)
\end{array}\right)\left(x_{i, A_{j}^{c}}, \frac{u_{i}-u}{h} x_{i, A_{j}^{c}}\right)\right\|_{F} \\
& =\sup _{l \in[k], j \in[p-k]}\left\{\left[\sum_{i} w_{i} f_{i}\left(\tilde{q}_{i}\right)\left(r_{i l}+x_{i, A^{c}}\left(\zeta_{l}^{\star}\left(u_{i}\right)-\zeta_{l}^{\star}(u)-\left(u_{i}-u\right) \cdot \nabla_{u} \zeta_{l}^{\star}(u)\right) \cdot x_{i, A_{j}^{c}}\right]^{2}\right.\right. \\
& +\left[\sum_{i} w_{i} f_{i}\left(\tilde{q}_{i}\right)\left(r_{i l}+x_{i, A^{c}}\left(\zeta_{l}^{\star}\left(u_{i}\right)-\zeta_{l}^{\star}(u)-\left(u_{i}-u\right) \cdot \nabla_{u} \zeta_{l}^{\star}(u)\right) \cdot \frac{u_{i}-u}{h} x_{i, A_{j}^{c}}\right]^{2}\right. \\
& +\left[\sum_{i} w_{i} f_{i}\left(\tilde{q}_{i}\right) \frac{u-u_{i}}{h}\left(r_{i l}+x_{i, A^{c}}\left(\zeta_{l}^{\star}\left(u_{i}\right)-\zeta_{l}^{\star}(u)\right)\right) \cdot x_{i, A_{j}^{c}}\right]^{2} \\
& \left.+\left[\sum_{i} w_{i} f_{i}\left(\tilde{q}_{i}\right) \frac{u-u_{i}}{h}\left(r_{i l}+x_{i, A^{c}}\left(\zeta_{l}^{\star}\left(u_{i}\right)-\zeta_{l}^{\star}(u)\right)\right) \cdot \frac{u_{i}-u}{h} x_{i, A_{j}^{c}}\right]^{2}\right\}^{-1 / 2} \\
& =O\left(\epsilon_{r}+h\right) \leq \lambda^{\star},
\end{aligned}
$$

we have that Assumption 5 holds.

\section{Acknowledgments}

We thank Rina Foygel Barber for numerous suggestions and detailed advice, as well as careful reading of various versions of the manuscript.

\section{References}

R. F. Barber and M. Kolar. Rocket: Robust confidence intervals via kendall's tau for transelliptical graphical models. Annals of Statistics, 46(6B):3422-3450, 2018. MR3852657

E. Belilovsky, G. Varoquaux, and M. B. Blaschko. Testing for differences in gaussian graphical models: Applications to brain connectivity. In Advances in Neural Information Processing Systems 29, pages 595-603, 2016.

A. Belloni and V. Chernozhukov. $\ell_{1}$-penalized quantile regression in highdimensional sparse models. Annals of Statistics, 39(1):82-130, 2011. MR2797841

A. Belloni and V. Chernozhukov. Least squares after model selection in highdimensional sparse models. Bernoulli, 19(2):521-547, 2013. MR3037163 
A. Belloni, V. Chernozhukov, and C. B. Hansen. Inference on treatment effects after selection amongst high-dimensional controls. The Review of Economic Studies, 81(2):608-650, 2013a. MR3207983

A. Belloni, V. Chernozhukov, and K. Kato. Valid post-selection inference in high-dimensional approximately sparse quantile regression models. Journal of the American Statistical Association, 114(526):749-758, 2019. MR3963177

A. Belloni, V. Chernozhukov, and K. Kato. Uniform post-selection inference for least absolute deviation regression and other Z-estimation problems. Biometrika, 102(1):77-94, 2015. MR3335097

A. Belloni, M. Chen, and V. Chernozhukov. Quantile graphical models: Prediction and conditional independence with applications to financial risk management. ArXiv e-prints, arXiv:1607.00286, 2016a.

A. Belloni, V. Chernozhukov, and Y. Wei. Post-selection inference for generalized linear models with many controls. Journal of Business and Economic Statistics, 34(4):606-619, 2016b. MR3547999

S. Boucheron, G. Lugosi, and P. Massart. Concentration inequalities. Oxford University Press, Oxford, 2013. MR3185193

J. Bradic and M. Kolar. Uniform inference for high-dimensional quantile regression: linear functionals and regression rank scores. ArXiv e-prints, arXiv:1702.06209, 2017.

V. H. de la Peña, T. L. Lai, and Q.-M. Shao. Self-normalized processes. SpringerVerlag, Berlin, 2009. MR2488094

J. Fan and W. Zhang. Simultaneous confidence bands and hypothesis testing in varying-coefficient models. Scandinavian Journal of Statistics, 27(4):715-731, 2000. MR1804172

M. H. Farrell. Robust inference on average treatment effects with possibly more covariates than observations. Journal of Econometrics, 189(1):1-23, 2015. MR3397349

E. Giné and A. Guillou. On consistency of kernel density estimators for randomly censored data: rates holding uniformly over adaptive intervals. Annales de l'Institut Henri Poincare (B) Probability and Statistics, 37(4):503-522, 2001. MR1876841

T. J. Hastie and R. J. Tibshirani. Varying-coefficient models. Journal of the Royal Statistical Society: Series B (Statistical Methodology), 55(4):757-796, 1993. MR1229881

D. R. Hoover, J. A. Rice, C. O. Wu, and L.-P. Yang. Nonparametric smoothing estimates of time-varying coefficient models with longitudinal data. Biometrika, 85(4):809-822, 1998. MR1666699

J. Z. Huang, C. O. Wu, and L. Zhou. Polynomial spline estimation and inference for varying coefficient models with longitudinal data. Statistica Sinica, 14(3):763-788, 2004. MR2087972

J. Janková and S. van de Geer. Confidence intervals for high-dimensional inverse covariance estimation. Electronic Journal of Statistics, 9(1):1205-1229, 2015. MR3354336

J. Janková and S. A. van de Geer. Honest confidence regions and optimality in high-dimensional precision matrix estimation. TEST, 26(1):143-162, 2017. 
MR3613609

A. Javanmard and A. Montanari. Hypothesis testing in high-dimensional regression under the gaussian random design model: Asymptotic theory. IEEE Transactions on Information Theory, 60(10):6522-6554, 2014.

A. Javanmard and A. Montanari. Nearly optimal sample size in hypothesis testing for high-dimensional regression. In 51st Annual Allerton Conference on Communication, Control, and Computing, 1427-1434, 2013.

B. Kai, R. Li, and H. H. Zhou. New efficient estimation and variable selection methods for semiparametric varying-coefficient partially linear models. Annals of Statistics, 39(1):305-332, 2011. MR2797848

B. Kim, S. Liu, and M. Kolar. Two-sample inference for high-dimensional markov networks. Journal of the Royal Statistical Society: Series B (Statistical Methodology), 83(5):939-962, 2021. MR4349123

M.-O. Kim. Quantile regression with varying coefficients. Annals of Statistics, 35(1):92-108, 2007. MR2332270

R. Koenker. A note on L-estimates for linear models. Statistics and Probability Letters, 2(6):323-325, 1984. MR0782652

R. Koenker. Quantile regression, volume 38 of Econometric Society Monographs. Cambridge University Press, Cambridge, 2005. MR2268657

V. Koltchinskii and M. Yuan. Sparsity in multiple kernel learning. Annals of Statistics, 38(6):3660-3695, 2010. MR2766864

D. Kozbur. Inference in additively separable models with a high dimensional set of conditioning variables. Journal of Business and Economic Statistics, 39:4, 984-1000, 2013. MR4319686

J. D. Lee, D. L. Sun, Y. Sun, and J. E. Taylor. Exact post-selection inference with the lasso. Annals of Statistics,44(3): 907-927, 2016.

W. Liu. Structural similarity and difference testing on multiple sparse Gaussian graphical models. Annals of Statistics, 45(6):2680-2707, 2017. MR3737906

R. Lockhart, J. E. Taylor, R. J. Tibshirani, and R. J. Tibshirani. A significance test for the lasso. Annals of Statistics, 42(2):413-468, 2014. MR3210970

J. Lu, M. Kolar, and H. Liu. Post-regularization inference for time-varying nonparanormal graphical models. Journal of Machine Learning Research, 18(203):1-78, 2018. MR3827091

J. Lu, M. Kolar, and H. Liu. Kernel meets sieve: Post-regularization confidence bands for sparse additive model. Journal of the American Statistical Association, 115(532):2084-2099, 2020. MR4189778

N. Meinshausen. Group bound: confidence intervals for groups of variables in sparse high dimensional regression without assumptions on the design. Journal of the Royal Statistical Society Series B (Statistical Methodology), 77(5):923-945, 2015. MR3414134

S. Na and M. Kolar. High-dimensional index volatility models via stein's identity. Bernoulli, 27(2):794-817, 2021. MR4255216

S. Na, Z. Yang, Z. Wang, and M. Kolar. High-dimensional varying index coefficient models via stein's identity. Journal of Machine Learning Research, 20(152):1-44, 2019. MR4030166

S. N. Negahban, P. Ravikumar, M. J. Wainwright, and B. Yu. A unified frame- 
work for high-dimensional analysis of $m$-estimators with decomposable regularizers. Statistical Science, 27(4):538-557, 2012. MR3025133

D. W. Nierenberg, T. A. Stukel, J. A. Baron, B. J. Dain, and E. R. Greenberg. Determinants of plasma levels of beta-carotene and retinol. American Journal of Epidemiology, 130(3):511-521, 1989.

D. Nolan and D. Pollard. $U$-processes: rates of convergence. Annals of Statistics, 15(2):780-799, 1987. MR0888439

Z. Ren, T. Sun, C.-H. Zhang, and H. H. Zhou. Asymptotic normality and optimalities in estimation of large Gaussian graphical models. Annals of Statistics, 43(3):991-1026, 2015. MR3346695

T. Sun and C.-H. Zhang. Sparse matrix inversion with scaled lasso. Journal of Machine Learning Research, 14:3385-3418, 2013. MR3144466

Y. Tang, X. Song, H. J. Wang, and Z. Zhu. Variable selection in high-dimensional quantile varying coefficient models. Journal of Multivariate Analysis, 122:115132, 2013. MR3189311

J. E. Taylor, R. Lockhart, R. Tibshirani, and R. J. Tibshirani. Exact postselection inference for sequential regression procedures. Journal of the American Statistical Association, 111(514):600-620, 2016. MR3538689

R. J. Tibshirani. Regression shrinkage and selection via the lasso. Journal of the Royal Statistical Society Series B (Statistical Methodology), 58(1):267288, 1996. MR1379242

S. A. van de Geer and P. Bühlmann. $\ell_{0}$-penalized maximum likelihood for sparse directed acyclic graphs. Annals of Statistics, 41(2):536-567, 2013. MR3099113

S. A. van de Geer, P. Bühlmann, Y. Ritov, and R. Dezeure. On asymptotically optimal confidence regions and tests for high-dimensional models. Annals of Statistics, 42(3):1166-1202, 2014. MR3224285

A. W. van der Vaart and J. A. Wellner. Weak Convergence and Empirical Processes: With Applications to Statistics. Springer, 1996. MR1385671

H. J. Wang, Z. Zhu, and J. Zhou. Quantile regression in partially linear varying coefficient models. Annals of Statistics, 37(6B):3841-3866, 2009. MR2572445

J. Wang and M. Kolar. Inference for sparse conditional precision matrices. ArXiv e-prints, arXiv:1412.7638, 2014.

J. Wang and M. Kolar. Inference for high-dimensional exponential family graphical models. In 19th International Conference on Artificial Intelligence and Statistics, pages 1042-1050, 2016.

X. Wang, M. Kolar, and A. Shojaie. Statistical inference for networks of highdimensional point processes. ArXiv e-prints, arXiv:2007.07448, 2020.

Y. Xia, T. Cai, and T. T. Cai. Testing differential networks with applications to the detection of gene-gene interactions. Biometrika, 102(2):247-266, 2015. MR3371002

M. Yu, V. Gupta, and M. Kolar. Statistical inference for pairwise graphical models using score matching. In Advances in Neural Information Processing Systems 29, 2016.

M. Yu, V. Gupta, and M. Kolar. Constrained high dimensional statistical inference. ArXiv e-prints, arXiv:1911.07319, 2020a.

M. Yu, V. Gupta, and M. Kolar. Simultaneous inference for pairwise graph- 
ical models with generalized score matching. Journal of Machine Learning Research, 21(91):1-51, 2020b. MR4119159

C.-H. Zhang and S. S. Zhang. Confidence intervals for low dimensional parameters in high dimensional linear models. Journal of the Royal Statistical Society Series B (Statistical Methodology), 76(1):217-242, 2013. MR3153940

W. Zhang, S.-Y. Lee, and X. Song. Local polynomial fitting in semivarying coefficient model. Journal of Multivariate Analysis, 82(1):166-188, 2002. MR1918619

T. Zhao, M. Kolar, and H. Liu. A general framework for robust testing and confidence regions in high-dimensional quantile regression. ArXiv e-prints, arXiv:1412.8724, 2014. 